\title{
The vectorial Ribaucour transformation for submanifolds of constant sectional curvature
}

\author{
D. Guimarães and R. Tojeiro
}

\begin{abstract}
We obtain a reduction of the vectorial Ribaucour transformation that preserves the class of submanifolds of constant sectional curvature of space forms, which we call the $L$-transformation. It allows to construct a family of such submanifolds starting with a given one and a vector-valued solution of a system of linear partial differential equations. We prove a decomposition theorem for the $L$-transformation, which is a far-reaching generalization of the classical permutability formula for the Ribaucour transformation of surfaces of constant curvature in Euclidean three space. As a consequence, we derive a Bianchi-cube theorem, which allows to produce, from $k$ initial scalar $L$-transforms of a given submanifold of constant curvature, a whole $k$-dimensional cube all of whose remaining $2^{k}-(k+1)$ vertices are submanifolds with the same constant sectional curvature given by explicit algebraic formulae. We also obtain further reductions, as well as corresponding decomposition and Bianchicube theorems, for the classes of $n$-dimensional flat Lagrangian submanifolds of $\mathbb{C}^{n}$ and $n$-dimensional Lagrangian submanifolds with constant curvature $c$ of the complex projective space $\mathbb{C P}^{n}(4 c)$ or the complex hyperbolic space $\mathbb{C} \mathbb{H}^{n}(4 c)$ of complex dimension $n$ and constant holomorphic curvature 4c.
\end{abstract}

The study of isometric immersions of space forms into space forms is a central topic in submanifold theory, having its origins in the study of surfaces $f: M^{2}(c) \rightarrow \mathbb{Q}^{3}(\tilde{c})$ of constant Gauss curvature $c$ in three-dimensional space forms of constant sectional curvature $\tilde{c}$. If $c \neq 0$ (respectively, $c=0$ ), the Gauss and Codazzi equations of such surfaces reduce to the sin-Gordon or sinh-Gordon equations (respectively, wave and Laplace equations), depending on whether $c<\tilde{c}$ or $c>\tilde{c}$, respectively. Understanding the complicated structure of these equations has led to some well-known global nonexistence results as well as to beautiful transformation theories for the construction of local examples.

The algebraic structure of the second fundamental form of an isometric immersion $f: M^{n}(c) \rightarrow \mathbb{Q}^{n+p}(c)$ of higher dimension and codimension was investigated by E. Cartan by means of his theory of exteriorly orthogonal quadratic forms. He also studied isometric 
immersions $f: M^{n}(c) \rightarrow \mathbb{Q}^{n+p}(\tilde{c})$ with $c<\tilde{c}$ by looking at their compositions $i \circ f$ with an umbilical inclusion $i: \mathbb{Q}^{n+p}(\tilde{c}) \rightarrow \mathbb{Q}^{n+p+1}(c)$, and proved that for such an isometric immersion one must have $p \geq n-1$, and that for $p=n-1$ the normal bundle is flat.

Cartan's algebraic results were later extended to the dual case $c>\tilde{c}$ by Moore [12]. If $p \leq n-2$, it turns out that the only possible structure for the second fundamental form is that of a composition $f=h \circ i$ of an umbilical inclusion $i: M^{n}(c) \rightarrow \mathbb{Q}^{n+1}(\tilde{c})$ with an isometric immersion $h: U \subset \mathbb{Q}^{n+1}(\tilde{c}) \rightarrow \mathbb{Q}^{n+p}(\tilde{c})$ with $i\left(M^{n}(c)\right) \subset U$. If $p=n-1$, exactly one further possibility arises, in which case the immersion has also flat normal bundle.

Flatness of the normal bundle is thus a natural condition for isometric immersions $f: M^{n}(c) \rightarrow \mathbb{Q}^{n+p}(\tilde{c})$, and implies that $M^{n}(c)$ can be locally endowed with principal coordinates with respect to which the integrability conditions give rise to nonlinear partial differential equations that reduce to the wave, Laplace, sin and sinh-Gordon equations when $n=2, p=1$ and $c \neq \tilde{c}$ (see Propositions 1 and 2 below).

For the construction of explicit examples of submanifolds of constant sectional curvature of o space forms with higher dimension and codimension, a useful tool is the Ribaucour transformation developed in [6] (see also [7]) as an extension of the classical Ribaucour transformation for surfaces in Euclidean three space. It allows to produce a family of new submanifolds of constant sectional curvature of a space form starting with a given one and a solution of a linear system of partial differential equations.

Aiming at a better understanding of the iteration of Ribaucour transformations for submanifolds, with an eye towards deriving a procedure for the construction of all submanifolds with flat normal bundle, a vectorial version of the transformation was developed in [2], based on its version in [10] for orthogonal systems, which shed light on its permutability properties.

In this paper we obtain a reduction of the vectorial Ribaucour transformation that preserves the class of submanifolds of constant sectional curvature of space forms. It depends on a linear operator $L$ of a vector space $V$, so we call it the $L$ - Ribaucour transformation, or simply the $L$-transformation. In the scalar case, that is, when $V$ has dimension one, it reduces to the scalar Ribaucour transformation for submanifolds of constant sectional curvature studied in [6]. The $L$-transformation allows to construct a family of new submanifolds of constant sectional curvature starting with a given one and a vector-valued solution of a linear system of PDE's. Proving the existence of $L$-transforms of a submanifold of constant sectional curvature with given initial conditions requires looking for invertible solutions of a certain system of Sylvester-type matrix equations.

We prove a decomposition theorem for the $L$-transformation, which is a far-reaching generalization of the permutability formula for the Ribaucour transformation of surfaces of constant curvature in Euclidean three space. It implies that an $L$-transformation given by a diagonalizable operator $L$ is the iterate of $n=\operatorname{dim} V$ scalar Ribaucour transformations of the type considered in [6]. In particular, we show that $k$ such Ribaucour transforms 
of a given submanifold of constant sectional curvature give rise to a whole $k$-dimensional cube, all of whose remaining $2^{k}-(k+1)$ vertices are submanifolds with the same constant sectional curvature given by means of explicit algebraic formulae. On the other hand, an $L$-transformation given by a non-diagonalizable linear operator $L$ yields new submanifolds of constant sectional curvature that can not be obtained by an iteration of a sequence of scalar Ribaucour transformations as those in [6].

We develop a further reduction of the $L$-transformation that preserves the classes of $n$-dimensional flat Lagrangian submanifolds of $\mathbb{C}^{n}=\mathbb{R}^{2 n}$ and $n$-dimensional submanifolds with constant curvature $c$ of $\mathbb{S}_{\epsilon}^{2 n+1}(c)$ that are horizontal with respect to the Hopf fibration $\pi: \mathbb{S}_{\epsilon}^{2 n+1}(c) \rightarrow \tilde{M}^{n}(4 c)$. Here $\mathbb{S}_{\epsilon}^{2 n+1}(c)$ stands for either the standard Euclidean sphere or the anti-de-Sitter space time of dimension $2 n+1$ and constant curvature $c$, corresponding to $\epsilon=1$ or $\epsilon=-1$, respectively, and $\tilde{M}^{n}(4 c)$ denotes either the complex projective space $\mathbb{C P}^{n}(4 c)$ or complex hyperbolic space $\mathbb{C H}^{n}(4 c)$ of complex dimension $n$ and constant holomorphic sectional curvature $4 \mathrm{c}$, corresponding to $c>0$ or $c<0$, respectively. Horizontal $n$-dimensional submanifolds with constant curvature $c$ of $\mathbb{S}_{\epsilon}^{2 n+1}(c)$ project down to $n$-dimensional Lagrangian submanifolds with constant curvature $c$ of $\tilde{M}^{n}(4 c)$.

The new transformation also depends on an operator $P$ of a vector space $V$, so we call it the $P$-transformation. Proving the existence of $P$-transforms of a given submanifold satisfying certain initial conditions now requires investigating under which conditions a certain Lyapunov-type matrix equation admits invertible solutions. The $P$-transformation provides a process to construct a family of $n$-dimensional flat Lagrangian submanifolds of $\mathbb{C}^{n}$ and $n$-dimensional horizontal submanifolds with constant curvature $c$ of $\mathbb{S}_{\epsilon}^{2 n+1}(c)$ starting with a given one and a vector-valued solution of a linear system of partial differential equations.

We prove a decomposition result for the $P$-transformation and a corresponding Bianchi $P$-cube theorem, which shows how to generate a $k$-dimensional cube of $n$-dimensional flat Lagrangian submanifolds of $\mathbb{C}^{n}$ (respectively, $n$-dimensional horizontal submanifolds with constant sectional curvature $c$ of $\left.\mathbb{S}_{\epsilon}^{2 n+1}(c)\right)$ starting with $k$ such submanifolds. As before, the remaining $2^{k}-(k+1)$ vertices of the cube are given by explicit algebraic formulae. We also obtain a further reduction of the $P$-transformation, as well as corresponding decomposition and Bianchi-cube theorems, that preserves the class of $n$-dimensional flat Lagrangian submanifolds of $\mathbb{R}^{2 n}$ that are contained in $\mathbb{S}^{2 n-1}$. These are the lifts by the Hopf projection $\pi: \mathbb{S}^{2 n-1} \rightarrow \mathbb{C P}^{n-1}$ of $(n-1)$-dimensional flat Lagrangian submanifolds of $\mathbb{C P}^{n-1}$.

We illustrate the procedures in the paper by applying the $P$-transformation to the vacuum solution of the system of partial differential equations associated to $n$-dimensional flat Lagrangian submanifolds of $\mathbb{C}^{n}$. First, starting with scalar $P_{i}$-transforms, $1 \leq i \leq k$, of the associated (degenerate) submanifold (which are themselves immersed submanifolds), with $P_{i} \neq \pm P_{j}$ for $1 \leq i \neq j \leq k$, we write down the formulae for the remaining 
$2^{k}-(k+1)$ vertices of the corresponding Bianhi $P$-cube. We also produce an example of an $n$-dimensional flat Lagrangian submanifold of $\mathbb{C}^{n}$ by applying the $P$-transformation to the vacuum solution with a non-diagonalizable $P$, which therefore can not be produced by an iteration of scalar $P$-transformations.

We point out that the vectorial Ribaucour transformation of flat Lagrangian submanifolds of $\mathbb{C}^{n}$ and $\mathbb{C P}^{n-1}$ was also studied in [15] under a different approach based on the dressing action of a rational loop group on these immersions.

\section{Preliminaries}

In this section we recall some known results on systems of partial differential equations associated to isometric immersions of space forms into space forms, as well as on the Ribaucour transformation and its vectorial version. Since our study of Lagrangian submanifolds of constant curvature $c$ of $\mathbb{C H}^{n}(4 c)$ requires working on Euclidean space with a flat metric of index two, we consider pseudo-Riemannian ambient space forms.

\subsection{PDE's associated to submanifolds of constant curvature}

Given an isometric immersion $f: M^{n} \rightarrow \mathbb{Q}_{s}^{n+p}(c)$ of a Riemannian manifold into a pseudoRiemannian space form of constant sectional curvature $c$ and index $s$, we denote by $N_{1}^{f}(x)$ the first normal space of $f$ at $x \in M^{n}$, which is the subspace of the normal space $N_{f} M(x)$ of $f$ at $x$ spanned by the image of the second second fundamental form $\alpha_{f}$ at $x$. We say that $N_{1}^{f}(x)$ is nondegenerate if $N_{1}^{f}(x) \cap N_{1}^{f}(x)^{\perp}=\{0\}$. We also denote by $\nu_{f}(x)$ the index of relative nullity of $f$ at $x$, defined as the dimension of the kernel of $\alpha_{f}$ at $x$.

The following results are well known (cf. [16] and [6]).

Proposition 1 Let $f: M^{n}(c) \rightarrow \mathbb{Q}_{s}^{n+p}(c)$ be an isometric immersion with flat normal bundle and $\nu_{f} \equiv 0$. If $s \geq 1$, suppose further that $N_{1}^{f}(x)$ is nondegenerate everywhere. Then $p \geq n$ and there exist locally principal coordinates $\left(u_{1}, \ldots, u_{n}\right)$, an orthonormal normal frame $\xi_{1}, \ldots, \xi_{p}$ and smooth functions $v_{1}, \ldots, v_{n}$ and $h_{i r}, 1 \leq i \leq n, 1 \leq r \neq i \leq p$, with $v_{1}, \ldots, v_{n}$ positive, such that

$$
d s^{2}=\sum_{j} v_{j}^{2} d u_{j}^{2}, \quad \alpha\left(\partial_{i}, \partial_{j}\right)=v_{i} \delta_{i j} \xi_{i}, \quad \nabla_{\partial_{i}} X_{j}=h_{j i} X_{i} \quad \text { and } \quad \nabla_{\partial_{i}}^{\perp} \xi_{r}=h_{i r} \xi_{i}
$$

where $\partial_{i}=\frac{\partial}{\partial u_{i}}, X_{i}=\left(1 / v_{i}\right) \partial_{i}$ and $h_{i j}=\left(1 / v_{i}\right) \partial_{i} v_{j}$ for $1 \leq i \neq j \leq n$. Moreover, the pair $(v, h)$, where $v=\left(v_{1}, \ldots, v_{n}\right)$ and $h=\left(h_{i r}\right)$, satisfies the system of PDE's

$$
\begin{cases}\text { i) } \partial_{j} v_{i}=h_{j i} v_{j}, & \text { ii) } \partial_{i} h_{i j}+\partial_{j} h_{j i}+\sum_{\ell=1}^{n} h_{\ell i} h_{\ell j}+c v_{i} v_{j}=0, \\ \text { iii) } \partial_{j} h_{i r}=h_{i j} h_{j r}, & \text { iv) } \epsilon_{j} \partial_{j} h_{i j}+\epsilon_{i} \partial_{i} h_{j i}+\sum_{r=1}^{p} \epsilon_{r} h_{i r} h_{j r}=0,\end{cases}
$$


where always $i \neq j,\{\ell, r\} \cap\{i, j\}=\emptyset$ and $\epsilon_{r}=\left\langle\xi_{r}, \xi_{r}\right\rangle$.

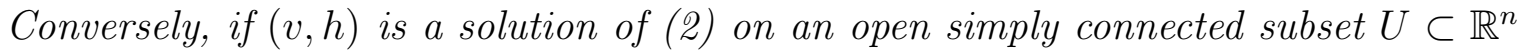
such that $v_{i}>0$ everywhere then there exists an immersion $f: U \rightarrow \mathbb{Q}_{s}^{n+p}(c)$ with flat normal bundle, $\nu_{f} \equiv 0$, nondegenerate first normal bundle of rank $n$ and induced metric $d s^{2}=\sum_{i} v_{i}^{2} d u_{i}^{2}$ of constant sectional curvature $c$.

In the next proposition, $\mathbb{O}_{s}^{t}(n \times p)$ stands for the subspace of $M_{n \times p}(\mathbb{R})$ of all matrices $V$ that satisfy $V^{t} J V=\tilde{J}$, where $J_{i j}=\epsilon_{i} \delta_{i j}$ and $\tilde{J}_{i j}=\tilde{\epsilon}_{i} \delta_{i j}$, the $\epsilon_{i}$ being -1 for $s$ of the indices $1, \ldots, p$ and 1 for the others, and the $\tilde{\epsilon}_{i}$ being -1 for $t$ of the indices $1, \ldots, n$ and 1 for the others.

Proposition 2 Let $f: M^{n}(c) \rightarrow \mathbb{Q}_{s}^{n+p}(\widetilde{c}), c \neq \tilde{c}$, be an isometric immersion with flat normal bundle. Let $X_{1}, \ldots, X_{n}$ be an orthonormal frame that diagonalizes the second fundamental form of $f$ and let $\eta_{i}=\alpha_{f}\left(X_{i}, X_{i}\right), 1 \leq i \leq n$, be the principal normal vector fields of $f$. Assume that

$$
\theta_{i}=\left\langle\eta_{i}, \eta_{i}\right\rangle+\tilde{c}-c \neq 0
$$

for $1 \leq i \leq n$. Then $p \geq n-1$ and there exist locally principal coordinates $u_{1}, \ldots, u_{n}$ on $M^{n}(c)$ for $f$. Moreover, if $\xi_{1}, \ldots, \xi_{p}$ is a parallel orthonormal frame of $N_{f} M$ and $V_{i r}$, $1 \leq i \leq n, 1 \leq r \leq p$, are defined by

$$
A_{\xi_{r}} X_{i}=v_{i}^{-1} V_{i r} X_{i},
$$

where $v_{i}=\left|\partial_{i}\right|$ for $1 \leq i \leq n$, then the triple $(v, h, V)$, with $v=\left(v_{1}, \ldots, v_{n}\right), h=\left(h_{i j}\right)$ for $h_{i j}=\left(1 / v_{i}\right) \partial_{i} v_{j}, 1 \leq i \neq j \leq n$, and $V=\left(V_{i r}\right)$, satisfies the system of PDE's

$$
\begin{cases}\text { i) } \partial_{i} v_{j}=h_{i j} v_{i} & \text { ii) } \partial_{\ell} V_{i r}=h_{\ell i} V_{\ell r}, \\ \text { iii) } \partial_{i} h_{i j}+\partial_{j} h_{j i}+\sum_{\ell=1}^{n} h_{\ell i} h_{\ell j}+c v_{i} v_{j}=0, & \text { iv) } \partial_{j} h_{i \ell}=h_{i j} h_{j \ell},\end{cases}
$$

where $1 \leq i \neq j \neq \ell \neq i \leq n$. Furthermore, the matrix $\hat{V} \in M_{n \times(p+1)}(\mathbb{R})$ defined by

$$
\hat{V}_{i r}=V_{i r}, 1 \leq r \leq p, \text { and } \hat{V}_{i(p+1)}=\sqrt{|c-\tilde{c}|} v_{i}
$$

belongs to $\mathbb{O}_{s+\epsilon_{0}}^{t}(n \times(p+1))$, where $\epsilon_{0}=0$ or 1 according to whether $\tilde{c}>c$ or $\tilde{c}<c$, respectively, and $t$ is the number of indices for which $\theta_{i}<0$.

Conversely, if $(v, h, V)$ is a solution of (3) on an open simply connected subset $U \subset \mathbb{R}^{n}$ such that $v_{i} \neq 0$ everywhere and such that $\hat{V} \in M_{n \times(p+1)}(\mathbb{R})$, defined by (4) for $\tilde{c} \neq c$, belongs to $\mathbb{O}_{s+\epsilon_{0}}^{t}(n \times(p+1))$, then there exists an immersion $f: U \rightarrow \mathbb{Q}_{s}^{n+p}(\tilde{c})$, with $\theta_{i}<0$ for $t$ of the indices, that has $(v, h, V)$ as associated triple and whose induced metric $d s^{2}=\sum_{i} v_{i}^{2} d u_{i}^{2}$ has constant sectional curvature $c$. 


\subsection{The vectorial Ribaucour transformation}

If $M^{n}$ is an $n$-dimensional Riemannian manifold and $\xi$ is a pseudo-Riemannian vector bundle over $M^{n}$ endowed with a compatible connection $\nabla^{\xi}$, we denote by $\Gamma(\xi)$ the space of smooth sections of $\xi$. If $\zeta=\xi^{*} \otimes \eta=\operatorname{Hom}(\xi, \eta)$ is the tensor product of the vector bundles $\xi^{*}$ and $\eta$, where $\xi^{*}$ stands for the dual vector bundle of $\xi$ and $\eta$ is a pseudoRiemannian vector bundle over $M^{n}$, then $\nabla Z \in \Gamma\left(T^{*} M \otimes \zeta\right)$ is given by

$$
\left(\nabla_{X}^{\zeta} Z\right)(v)=\nabla_{X}^{\eta} Z(v)-Z\left(\nabla_{X}^{\xi} v\right)
$$

for all $X \in \mathfrak{X}(M)=\Gamma(T M), v \in \Gamma(\xi)$. For $Z \in \Gamma\left(\xi^{*} \otimes \eta\right)$, we define $Z^{t} \in \Gamma\left(\eta^{*} \otimes \xi\right)$ by

$$
\left\langle Z^{t}(u), v\right\rangle=\langle u, Z(v)\rangle, \quad u \in \Gamma(\eta), v \in \Gamma(\xi) .
$$

The exterior derivative $d \omega \in \Gamma\left(\Lambda^{2} T^{*} M \otimes \xi\right)$ of $\omega$ is related to its covariant derivative by

$$
d \omega(X, Y)=\nabla \omega(X, Y)-\nabla \omega(Y, X)=\left(\nabla_{X} \omega\right) Y-\left(\nabla_{Y} \omega\right) X .
$$

The one-form $\omega$ is closed if $d \omega=0$. If $Z \in \Gamma(\xi)$, then $\nabla Z=d Z \in \Gamma\left(T^{*} M \otimes \xi\right)$ is the one-form given by $\nabla Z(X)=\nabla_{X}^{\xi} Z$. In case $\xi=M \times V$ is a trivial vector bundle over $M^{n}$, with $V$ a Euclidean vector space, that is, a vector space endowed with an inner product, then $\Gamma\left(T^{*} M \otimes \xi\right)$ is identified with the space of smooth one-forms with values in $V$.

The vectorial Ribaucour transformation for Euclidean submanifolds was introduced in 2], and can be extended to submanifolds of $\mathbb{R}_{s}^{n+p}$ as follows.

Let $f: M^{n} \rightarrow \mathbb{R}_{s}^{n+p}$ be an isometric immersion of a simply connected Riemannian manifold. Let $V$ be a vector space and let $\varphi: M^{n} \rightarrow V$ be a smooth map, which we regard as a section of the trivial vector bundle $M^{n} \times V$, also denoted by $V$. Fix an inner product $\langle\cdot, \cdot\rangle$ on $V$, thus making $M \times V$ into a Riemannian vector bundle, which we endow with the trivial compatible connection $\nabla=d$. Assume that $\beta \in \Gamma\left(V^{*} \otimes N_{f} M\right)$ is such that

$$
\alpha\left(X, \omega^{t}(v)\right)+\left(\nabla_{X} \beta\right) v=0
$$

for all $v \in \Gamma(V)$, where $\omega=d \varphi \in \Gamma\left(T M^{*} \otimes V\right)$, and

$$
\left[\Phi_{u}, \Phi_{v}\right]=0
$$

for all $u, v \in \Gamma(V)$, where $\Phi=\Phi(\omega, \beta) \in \Gamma\left(T^{*} M \otimes V^{*} \otimes T M\right)$ is given by

$$
\Phi_{v} X:=\Phi(X)(v)=\left(\nabla_{X} \omega^{t}\right) v-A_{\beta(v)} X .
$$

It follows from (5) that $\mathcal{F} \in \Gamma\left(V^{*} \otimes f^{*} T \mathbb{R}_{s}^{n+p}\right)$ given by $\mathcal{F}=f_{*} \omega^{t}+\beta$ satisfies

$$
d \mathcal{F}(X)(v)=f_{*} \Phi_{v} X
$$


for all $X \in \mathfrak{X}(M)$ and $v \in \Gamma(V)$. Now let $\Omega \in \Gamma\left(V^{*} \otimes V\right)$ be a solution of the completely integrable first order system

$$
d \Omega=\mathcal{F}^{t} d \mathcal{F}
$$

such that

$$
\Omega+\Omega^{t}=\mathcal{F}^{t} \mathcal{F} .
$$

We point out that the integrability conditions of (9) are precisely equations (6) (see [2]), and that (9) implies (10) up to a parallel section of $V^{*} \otimes V$.

Definition 3. If $\Omega$ is invertible everywhere, then the isometric immersion $\tilde{f}: \tilde{M}^{n} \rightarrow \mathbb{R}_{s}^{n+p}$ given by

$$
\tilde{f}=f-\mathcal{F} \Omega^{-1} \varphi
$$

where $\tilde{M}^{n}$ is the subset of regular points of $\tilde{f}$ endowed with the induced metric, is called the vectorial Ribaucour transform of $f$ determined by $(\varphi, \beta, \Omega)$ (or, more precisely, by $(\varphi, \beta, \Omega,\langle\cdot, \cdot\rangle)$; see Remark 4 below $)$. We write $\tilde{f}=\mathcal{R}_{\varphi, \beta, \Omega}(f)$.

Remark 4. Let $\langle\cdot, \cdot\rangle^{\sim}$ be another inner product on $V$, related to $\langle\cdot, \cdot\rangle$ by $\langle\cdot, \cdot\rangle^{\sim}=\langle\cdot, B \cdot\rangle$ for some invertible $B \in V^{*} \otimes V$, which we regard as a parallel section of the trivial vector bundle $V^{*} \otimes V$,

$$
\nabla_{X} B v=B \nabla_{X} v
$$

for all $X \in \mathfrak{X}(M)$ and $v \in \Gamma(V)$. The transpose $\hat{\nu}$ of $\omega=d \varphi$ with respect to $\langle\cdot, \cdot\rangle^{\sim}$ is related to its transpose $\nu=\omega^{t}$ with respect to $\langle\cdot, \cdot\rangle$ by $\hat{\nu}=\nu B$ and, in view of (12), also the covariant derivatives $\nabla \hat{\nu}$ and $\nabla \nu$ are related by

$$
\left(\nabla_{X} \hat{\nu}\right) v=\left(\nabla_{X} \nu\right) B v
$$

for all $X \in \mathfrak{X}(M)$ and $v \in \Gamma(V)$. Thus, if we define $\hat{\beta} \in \Gamma\left(V^{*} \otimes N_{f} M\right)$ by $\hat{\beta}=\beta B$, then

$$
\hat{\mathcal{F}}=f_{*} \hat{\nu}+\hat{\beta}=\mathcal{F} B
$$

and

$$
\hat{\Phi}_{v} X=\left(\hat{\nabla}_{X} \hat{\nu}\right) v-A_{\hat{\beta}(v)} X=\Phi_{B v} X .
$$

Hence (5) is satisfied by $(\hat{\nu}, \hat{\beta})$, and

$$
\left[\hat{\Phi}_{u}, \hat{\Phi}_{v}\right]=\left[\Phi_{B u}, \Phi_{B v}\right]=0
$$

for all $u, v \in \Gamma(V)$. Note also that the transposes $\hat{\beta}^{t}$ and $\beta^{t}$ with respect to $\langle\cdot, \cdot\rangle^{\sim}$ and $\langle\cdot, \cdot\rangle$, respectively, coincide, for

$$
\left\langle\hat{\beta}^{t} \xi, v\right\rangle^{\sim}=\langle\xi, \hat{\beta} v\rangle=\langle\xi, \beta B v\rangle=\left\langle\beta^{t} \xi, B v\right\rangle=\left\langle\beta^{t} \xi, v\right\rangle^{\sim} .
$$


Similarly, $\hat{\mathcal{F}}^{t}=\mathcal{F}^{t}$, and hence $\hat{\Omega} \in \Gamma\left(V^{*} \otimes V\right)$, given by $\hat{\Omega}=\Omega B$, satisfies $\hat{\Omega}^{t}=\Omega^{t} B$,

$$
d \hat{\Omega}=\hat{\mathcal{F}}^{t} d \hat{\mathcal{F}} \text { and } \hat{\Omega}+\hat{\Omega}^{t}=\hat{\mathcal{F}}^{t} \hat{\mathcal{F}} .
$$

Moreover,

$$
f-\hat{\mathcal{F}} \hat{\Omega}^{-1} \varphi=f-(\mathcal{F} B)\left(B^{-1} \Omega^{-1}\right) \varphi=f-\mathcal{F} \Omega^{-1} \varphi
$$

and thus $(\varphi, \beta, \Omega,\langle\cdot, \cdot\rangle)$ and $\left(\hat{\varphi}=\varphi, \hat{\beta}, \hat{\Omega},\langle\cdot, \cdot\rangle^{\sim}\right)$ give rise to the same vectorial Ribaucour transform.

If $\operatorname{dim} V=1$, after identifying $V^{*} \otimes N_{f} M$ with $N_{f} M$ then $\varphi$ and $\beta$ become elements of $C^{\infty}(M)$ and $\Gamma\left(N_{f} M\right)$, respectively, and equation (5) reduces to

$$
\alpha(X, \nabla \varphi)+\nabla_{X}^{\perp} \beta=0 .
$$

Moreover, $\Omega$ reduces to the function $\Omega=(1 / 2)\langle\mathcal{F}, \mathcal{F}\rangle$, where $\mathcal{F}=f_{*} \nabla \varphi+\beta$ is the Combescure transform of $f$ determined by $(\varphi, \beta)$, and (11) becomes the parameterization of a scalar Ribaucour transform of $f$ obtained in Theorem 17 of [7]. In this case, since $\Omega$ is determined by $\varphi$ and $\beta$ we write $\tilde{f}=\mathcal{R}_{\varphi, \beta}(f)$ instead of $\tilde{f}=\mathcal{R}_{\varphi, \beta, \Omega}(f)$. The tensor $\Phi=\Phi_{\varphi, \beta}$ given by (7) reduces in this case to

$$
\Phi=\operatorname{Hess} \varphi-A_{\beta}
$$

and is a Codazzi tensor on $M^{n}$ that is related to $\mathcal{F}$ by $\mathcal{F}_{*} X=f_{*} \Phi(X)$ for all $X \in \mathfrak{X}(M)$. It is called the Codazzi tensor associated to the Ribaucour transform $\tilde{f}$.

Given two pairs $(\varphi, \beta)$ and $\left(\varphi^{\prime}, \beta^{\prime}\right)$ satisfying (13) such that $\left[\Phi_{\varphi^{\prime}, \beta^{\prime}}, \Phi_{\varphi, \beta}\right]=0$, taking linear combinations $(\tilde{\varphi}, \tilde{\beta})=c(\varphi, \beta)+c^{\prime}\left(\varphi^{\prime}, \beta^{\prime}\right), c, c^{\prime} \in \mathbb{R}$, yields a one-parameter family of scalar Ribaucour transforms $\mathcal{R}_{\tilde{\varphi}, \tilde{\beta}}(f)$ of $f$, because pairs $(\varphi, \beta)$ and $\left(\varphi^{\prime}, \beta^{\prime}\right)$ related by $(\varphi, \beta)=\lambda\left(\varphi^{\prime}, \beta^{\prime}\right)$ for some $\lambda \neq 0$ give rise to the same Ribaucour transform. It is called the associated family determined by $\mathcal{R}_{\varphi, \beta}(f)$ and $\mathcal{R}_{\varphi^{\prime}, \beta^{\prime}}(f)$.

The vectorial Ribaucour transformation can be extended to isometric immersions $f: M^{n} \rightarrow \mathbb{Q}_{s}^{n+p}(\tilde{c})$ of a Riemannian manifold $M^{n}$ into a pseudo-Riemannian manifold of constant sectional curvature $\tilde{c}$ as follows. Here, and in the sequel, $i$ stands for the umbilical inclusion $i: \mathbb{Q}_{s}^{n+p}(\tilde{c}) \rightarrow \mathbb{R}_{s+\epsilon_{0}}^{n+p+1}$, where $\epsilon_{0}=0$ or $\epsilon_{0}=1$ depending on whether $\tilde{c}>0$ or $\tilde{c}<0$, respectively.

Definition 5 An isometric immersion $\tilde{f}: \tilde{M}^{n} \rightarrow \mathbb{Q}_{s}^{n+p}(\tilde{c})$ is said to be the vectorial Ribaucour transform of $f: M^{n} \rightarrow \mathbb{Q}_{s}^{n+p}(\tilde{c})$ with data $(\varphi, \beta, \Omega)$ if $\tilde{F}=i \circ \tilde{f}: \tilde{M}^{n} \rightarrow \mathbb{R}_{s+\epsilon_{0}}^{n+p+1}$ is a vectorial Ribaucour transform of $F=i \circ f: M^{n} \rightarrow \mathbb{R}_{s+\epsilon_{0}}^{n+p+1}$ with data $(\varphi, \tilde{\beta}, \Omega)$, where

$$
\tilde{\beta}=i_{*} \beta+\tilde{c} F \varphi^{t} .
$$


In this case, the corresponding vectorial Combescure transform of $F$ is

$$
\mathcal{G}=F_{*} \omega^{t}+i_{*} \beta+\tilde{c} F \varphi^{t}
$$

and the tensor $\Phi=\Phi(\omega, \beta)$ is now given by

$$
\Phi_{v} X:=\Phi(X)(v)=\left(\nabla_{X} \omega^{t}\right) v-A_{\beta(v)} X+\tilde{c} \varphi^{t}(v) X .
$$

Equations (9) and (10) become

$$
d \Omega=\mathcal{G}^{t} d \mathcal{G}
$$

and

$$
\Omega+\Omega^{t}=\mathcal{G}^{t} \mathcal{G}
$$

respectively, and (15) is equivalent to

$$
d \Omega=\omega \Phi .
$$

The following basic properties of the vectorial transformation were derived in [2] when $\tilde{c}=0$. The extension to the case $\tilde{c} \neq 0$ is straightforward.

Proposition 6. (i) The bundle map

$$
\mathcal{P}=I-\mathcal{G} \Omega^{-1} \mathcal{G}^{t} \in \Gamma\left(\left(F^{*} T \mathbb{R}_{s+\epsilon_{0}}^{n+p+1}\right)^{*} \otimes \tilde{F}^{*} T \mathbb{R}_{s+\epsilon_{0}}^{n+p+1}\right)
$$

is a vector bundle isometry satisfying

$$
\mathcal{P} F=\tilde{F}=F-\mathcal{G} \Omega^{-1} \varphi \text { and } \tilde{f}_{*}=\mathcal{P} f_{*} D
$$

where

$$
D=I-\Phi_{\Omega^{-1} \varphi} \in \Gamma\left(T^{*} M \otimes T M\right) .
$$

In particular, the metrics induced by $f$ and $\tilde{f}$ are related by $\langle,\rangle^{\sim}=D^{*}\langle$,$\rangle .$

(ii) The normal connections and second fundamental forms of $f$ and $\tilde{f}$ are related by

$$
\tilde{\nabla}_{X}^{\perp} \mathcal{P} \xi=\mathcal{P} \nabla_{X}^{\perp} \xi
$$

and

$$
\tilde{A}_{\mathcal{P} \xi}=D^{-1}\left(A_{\xi}+\Phi_{\Omega^{-1} \beta^{t} \xi}\right)
$$

or equivalently,

$$
\tilde{\alpha}(X, Y)=\mathcal{P}\left(\alpha(X, D Y)+\beta\left(\Omega^{-1}\right)^{t} \Phi(X)^{t} D Y\right) .
$$

(iii) The Levi-Civita connections of the metrics $\langle$,$\rangle and \langle,\rangle^{\sim}$ are related by

$$
D \tilde{\nabla}_{X} Y=\nabla_{X} D Y-\left(\Phi(X) \Omega^{-1} \omega-\left(\Phi(X) \Omega^{-1} \omega\right)^{t}\right)(D Y) .
$$




\subsection{The decomposition theorem}

The following is a straightforward extension for nonflat ambient space forms of a decomposition property of the vectorial Ribaucour transformation proved in [2] for Euclidean submanifolds.

Theorem 7 Let $\mathcal{R}_{\varphi, \beta, \Omega}(f): \tilde{M}^{n} \rightarrow \mathbb{Q}_{s}^{n+p}(\tilde{c})$ be a vectorial Ribaucour transform of an isometric immersion $f: M^{n} \rightarrow \mathbb{Q}_{s}^{n+p}(\tilde{c})$ determined by $(\varphi, \beta, \Omega)$ as in Definition 5. For a direct sum decomposition $V=V_{1} \oplus V_{2}$, which we assume to be orthogonal after a suitable change of the inner product on $V$ (see Remark Q), let $\pi_{j}: V \rightarrow V_{j}, 1 \leq j \leq 2$, denote the orthogonal projection and define

$$
\varphi_{j}=\pi_{V_{j}} \circ \varphi, \quad \beta_{j}=\left.\beta\right|_{V_{j}} \text { and } \Omega_{i j}=\left.\pi_{V_{i}} \circ \Omega\right|_{V_{j}} \in \Gamma\left(V_{j}^{*} \otimes V_{i}\right), \quad 1 \leq i, j \leq 2 .
$$

Then the triple $\left(\varphi_{j}, \beta_{j}, \Omega_{j j}\right)$ satisfies the conditions of Definition 5 for $1 \leq j \leq 2$, except possibly for the invertibility of $\Omega_{j j}$. Suppose that the latter condition is also satisfied and define $f_{j}=\mathcal{R}_{\varphi_{j}, \beta_{j}, \Omega_{j j}}(f), F_{j}=i \circ f_{j}, \mathcal{G}_{j}=F_{j_{*}} \omega_{j}^{t}+i_{*} \beta_{j}+\tilde{c} F_{j} \varphi_{j}^{t}$, where $\omega_{j}=d \varphi_{j}$, and

$$
\bar{\varphi}_{i}=\varphi_{i}-\Omega_{i j} \Omega_{j j}^{-1} \varphi_{j}, \quad \bar{\beta}_{i}=\mathcal{P}_{j}\left(\beta_{i}-\beta_{j}\left(\Omega_{j j}^{-1}\right)^{t} \Omega_{i j}^{t}\right) \quad \text { and } \quad \bar{\Omega}_{i i}=\Omega_{i i}-\Omega_{i j} \Omega_{j j}^{-1} \Omega_{j i},
$$

$1 \leq i \neq j \leq 2$, where $\mathcal{P}_{j}=I-\mathcal{G}_{j} \Omega_{j j}^{-1} \mathcal{G}_{j}^{t}$. Then the triple $\left(\bar{\varphi}_{i}, \bar{\beta}_{i}, \bar{\Omega}_{i i}\right)$ satisfies the conditions of Definition 5 with respect to $f_{j}, 1 \leq j \neq i \leq 2$, and

$$
\mathcal{R}_{\varphi, \beta, \Omega}(f)=\mathcal{R}_{\bar{\varphi}_{i}, \bar{\beta}_{i}, \bar{\Omega}_{i i}}\left(\mathcal{R}_{\varphi_{j}, \beta_{j}, \Omega_{j j}}(f)\right) .
$$

Theorem 7 implies that any vectorial Ribaucour transformation whose associated data $(\varphi, \beta, \Omega)$ are defined on a vector space $V$ can be regarded as the iteration of $k=\operatorname{dim} V$ scalar Ribaucour transformations. We discuss below a consequence of this fact.

The isometric immersions $f_{i}: M_{i}^{n} \rightarrow \mathbb{Q}_{s}^{n+p}(\tilde{c}), 1 \leq i \leq 4$, form a Bianchi quadrilateral if for each of them the preceding and subsequent ones (thought of as points on an oriented circle) are Ribaucour transforms of it and the associated Codazzi tensors commute.

A Bianchi cube is a $(k+1)$-tuple $\left(\mathcal{C}_{0}, \ldots, \mathcal{C}_{k}\right)$, where each $\mathcal{C}_{r}, 1 \leq r \leq k$, is a family of isometric immersions $f_{\alpha_{r}}: M_{\alpha_{r}}^{n} \rightarrow \mathbb{Q}_{s}^{n+p}(\tilde{c})$ indexed in the set of multi-indices

$$
\Lambda_{r}=\left\{\alpha_{r}=\left\{i_{1}, \ldots, i_{r}\right\} \subset\{1, \ldots, k\}: \alpha_{r} \text { with } r \text { distinct elements }\right\}
$$

satisfying the following conditions for all $1 \leq s \leq k-1$ :

(i) Each $f_{\alpha_{s+1}} \in \mathcal{C}_{s+1}$ with $\alpha_{s+1}=\alpha_{s} \cup\left\{i_{j}\right\}$ is a Ribaucour transform of $f_{\alpha_{s}} \in \mathcal{C}_{s}$.

(ii) $\left\{f_{\alpha_{s-1}}, f_{\alpha_{s-1} \cup\left\{i_{l}\right\}}, f_{\alpha_{s-1} \cup\left\{i_{j}\right\}}, f_{\alpha_{s+1}}\right\}$ is a Bianchi quadrilateral if $\alpha_{s+1}=\alpha_{s-1} \cup\left\{i_{l}, i_{j}\right\}$. 
Theorem 2 in [2] can be easily extended to nonflat ambient space forms as follows.

Theorem 8 Let $f: M^{n} \rightarrow \mathbb{Q}_{s}^{n+p}(\tilde{c})$ be an isometric immersion and let $f_{1}, \ldots, f_{k}$ be Ribaucour transforms of $f$ none of which belongs to the associated family determined by any two of the others. Then, for a generic choice of isometric immersions $f_{i j}: M_{i j}^{n} \rightarrow \mathbb{Q}_{s}^{n+p}(\tilde{c})$, $1 \leq i \neq j \leq k$, such that $\left\{f, f_{i}, f_{j}, f_{i j}\right\}$ is a Bianchi quadrilateral, there exists a unique Bianchi $k$-cube $\left(\mathcal{C}_{0}, \ldots, \mathcal{C}_{k}\right)$ such that $\mathcal{C}_{0}=\{f\}, \mathcal{C}_{1}=\left\{f_{1}, \ldots, f_{k}\right\}$ and $\mathcal{C}_{2}=\left\{f_{i j}\right\}_{1 \leq i \neq j \leq k}$.

\section{The $L$-transformation}

In this section we obtain a reduction of the vectorial Ribaucour transformation that preserves the class of submanifolds with constant curvature. First we relate the curvature tensors of submanifolds that are associated by a vectorial Ribaucour transformation.

Let $f: M^{n} \rightarrow \mathbb{Q}_{s}^{n+p}(\tilde{c})$ be an isometric immersion and let $\tilde{f}=\mathcal{R}_{\varphi, \beta, \Omega}(f): \tilde{M}^{n} \rightarrow$ $\mathbb{Q}_{s}^{n+p}(\tilde{c})$ be a vectorial Ribaucour transform of $f$. Set $S=\Omega^{-1} \beta^{t} \in \Gamma\left(N_{f} M^{*} \otimes V\right)$ and define $U \in \Gamma\left(T^{*} M \otimes T^{*} M \otimes T^{*} M \otimes T M\right)$ by

$$
U(X, Y)=\Phi(X) S\left(A(Y)+\frac{1}{2} \Phi(Y) S\right)^{t} .
$$

Given $T \in \Gamma\left(T^{*} M \otimes T^{*} M \otimes T^{*} M \otimes T M\right)$, define $\hat{T} \in \Gamma\left(\mathcal{A}_{2}\left(T^{*} M\right) \otimes \mathcal{A}(T M)\right)$ by

$$
\hat{T}(X, Y)=\left(T(X, Y)-T(X, Y)^{t}\right)-\left(T(Y, X)-T(Y, X)^{t}\right) .
$$

Proposition 9. The curvature tensors of $M^{n}$ and $\tilde{M}^{n}$ are related by

$$
D \tilde{R}(X, Y)=(R(X, Y)-\tilde{c}(X \wedge Y)+\hat{U}(X, Y)+\tilde{c}(D X \wedge D Y)) D .
$$

Proof: By (19) and (20) we have

$$
\begin{aligned}
D \tilde{A}_{\tilde{\alpha}(Y, Z)} X= & A(X)\left(\alpha(Y, D Z)+S^{t} \Phi(Y)^{t} D Z\right) \\
& +\Phi(X) S\left(\alpha(Y, D Z)+S^{t} \Phi(Y)^{t} D Z\right) .
\end{aligned}
$$

From (25) and the Gauss equations for $\tilde{f}$ and $f$ we obtain

$$
\begin{aligned}
D \tilde{R}(X, Y) Z=D & \tilde{A}_{\tilde{\alpha}(Y, Z)} X-D \tilde{A}_{\tilde{\alpha}(X, Z)} Y+D(\bar{R}(X, Y) Z)^{t} \\
= & R(X, Y) D Z-\tilde{c}(X \wedge Y) D Z+A(X) S^{t} \Phi(Y)^{t} D Z \\
& +\Phi(X) S A(Y)^{t} D Z+\Phi(X) S S^{t} \Phi(Y)^{t} D Z-A(Y) S^{t} \Phi(X)^{t} D Z \\
& -\Phi(Y) S A(X)^{t} D Z-\Phi(Y) S S^{t} \Phi(X)^{t} D Z+\tilde{c}(D X \wedge D Y) D Z \\
= & R(X, Y) D Z-\tilde{c}(X \wedge Y) D Z+\hat{U}(X, Y) D Z+\tilde{c}(D X \wedge D Y) D Z .
\end{aligned}
$$


Now define $\left.\rho \in \Gamma\left(V^{*} \otimes V\right), \Psi \in \Gamma\left(T^{*} M \otimes V^{*} \otimes T M\right)\right)$ and $Q \in \Gamma\left(T^{*} M \otimes T^{*} M \otimes T^{*} M \otimes T M\right)$ by

$$
\begin{gathered}
\rho=\beta^{t} \beta-(c-\tilde{c}) \varphi \varphi^{t}, \\
\Psi(Y)=A(Y) \beta+(c-\tilde{c}) Y \varphi^{t}+\frac{1}{2} \Phi(Y) \Omega^{-1} \rho
\end{gathered}
$$

and

$$
Q(X, Y)=\Phi(X) \Omega^{-1} \Psi(Y)^{t} .
$$

Corollary 10. If $M^{n}$ has constant sectional curvature $c$, then the same holds for $\tilde{M}^{n}$ if and only if $\hat{Q}$ vanishes identically.

Proof: First observe that $\tilde{M}^{n}$ has constant sectional curvature $c$ if and only if

$$
D \tilde{R}(X, Y) Z=c D\left(\langle Y, Z\rangle^{\sim} X-\langle X, Z\rangle^{\sim} Y\right)=c(D X \wedge D Y) D Z .
$$

By Proposition 9 and the fact that $M^{n}$ has constant sectional curvature $c$ we have

$$
\begin{aligned}
D \tilde{R}(X, Y)-c(D X \wedge D Y) D= & (R(X, Y)-\tilde{c}(X \wedge Y)+\hat{U}(X, Y) \\
& +\tilde{c}(D X \wedge D Y)-c(D X \wedge D Y)) D \\
= & ((c-\tilde{c})(X \wedge Y)-(c-\tilde{c})(D X \wedge D Y)+\hat{U}(X, Y)) D .
\end{aligned}
$$

Using that $D=I-\Phi_{\Omega^{-1} \varphi}$ we obtain

$$
\begin{aligned}
X \wedge Y-D X \wedge D Y= & X Y^{t}-Y X^{t}-D X(D Y)^{t}+D Y(D X)^{t} \\
= & X\left(\Phi(Y) \Omega^{-1} \varphi\right)^{t}+\left(\Phi(X) \Omega^{-1} \varphi\right) Y^{t}-\left(\Phi(X) \Omega^{-1} \varphi\right)\left(\Phi(Y) \Omega^{-1} \varphi\right)^{t} \\
& -Y\left(\Phi(X) \Omega^{-1} \varphi\right)^{t}-\left(\Phi(Y) \Omega^{-1} \varphi\right) X^{t}+\left(\Phi(Y) \Omega^{-1} \varphi\right)\left(\Phi(X) \Omega^{-1} \varphi\right)^{t} \\
& =\hat{H}(X, Y)
\end{aligned}
$$

where $H(X, Y)=\Phi(X) \Omega^{-1} \varphi\left(Y-\frac{1}{2} \Phi(Y) \Omega^{-1} \varphi\right)^{t}$. It follows from (29) and (301) that

$$
D \tilde{R}(X, Y)-c(D X \wedge D Y) D=(\hat{U}(X, Y)+(c-\tilde{c}) \hat{H}(X, Y)) D,
$$

and the proof is completed by checking that $U(X, Y)+(c-\tilde{c}) H(X, Y)=Q(X, Y)$.

Looking for triples $(\varphi, \beta, \Omega)$ for which $\hat{Q}=0$ leads to the following definition.

Definition 11. Let $\tilde{f}=\mathcal{R}_{\varphi, \beta, \Omega}(f): \tilde{M}^{n} \rightarrow \mathbb{Q}_{s}^{n+p}(\tilde{c})$ be a vectorial Ribaucour transform of $f: M^{n}(c) \rightarrow \mathbb{Q}_{s}^{n+p}(\tilde{c})$ determined by $(\varphi, \beta, \Omega)$, with $\varphi \in \Gamma(V), \beta \in \Gamma\left(V^{*} \otimes N_{f} M\right)$ and $\Omega \in \Gamma\left(V^{*} \otimes V\right)$. If there exists $L \in V^{*} \otimes V$ such that

$$
\Phi(Y) L+A(Y) \beta+(c-\widetilde{c}) Y \varphi^{t}=0
$$


where $\Phi=\Phi(\nu, \beta)$ is given by (14), and

$$
\Omega L+L^{t} \Omega^{t}=\rho
$$

with $\rho$ given by (26), then $\tilde{f}$ is said to be an L-vectorial Ribaucour transform of $f$, or simply an L-transform of $f$. We write $\tilde{f}=\mathcal{R}_{\varphi, \beta, \Omega, L}(f)$.

Remarks 12. (i) If $\langle\cdot, \cdot\rangle^{\sim}$ is another inner product on $V$, with $\langle\cdot, \cdot\rangle^{\sim}=\langle\cdot, B \cdot\rangle$ for some invertible $B \in V^{*} \otimes V$, then conditions (31) and (32) are satisfied by $(\varphi, \beta, \Omega, L,\langle\cdot, \cdot\rangle)$ if and only if they are satisfied by $\left(\hat{\varphi}, \hat{\beta}, \hat{\Omega}, \hat{L},\langle\cdot, \cdot\rangle^{\sim}\right)$, where $\hat{\varphi}=\varphi, \hat{\beta}=\beta B, \hat{\Omega}=\Omega B$ and $\hat{L}=$ $B^{-1} L B$. Thus, in the definition of the $L$-transformation one may replace $(\varphi, \beta, \Omega, L,\langle\cdot, \cdot\rangle)$ by any quintuple $\left(\hat{\varphi}, \hat{\beta}, \hat{\Omega}, \hat{L},\langle\cdot, \cdot\rangle^{\sim}\right)$ related to $(\varphi, \beta, \Omega, L,\langle\cdot, \cdot\rangle)$ in this way. Notice that the transposes of $\hat{L}$ and $L$ with respect to $\langle\cdot, \cdot\rangle^{\sim}$ and $\langle\cdot, \cdot\rangle$ coincide, for

$$
\left\langle\hat{L}^{t} v, w\right\rangle^{\sim}=\langle v, \hat{L} w\rangle^{\sim}=\langle v, B \hat{L} w\rangle=\langle v, L B w\rangle=\left\langle L^{t} v, B w\right\rangle=\left\langle L^{t} v, w\right\rangle^{\sim} .
$$

(ii) In the scalar case, the $L$-transformation reduces to the transformation for submanifolds of constant sectional curvature given by Theorem 13 of [6], called the $\mathcal{R}_{C^{-}}$ transformation in that paper, which in turn reduces to the Ribaucour transformation for constant curvature surfaces in $\mathbb{R}^{3}$ studied by Bianchi [1].

Theorem 13. If $f: M^{n}(c) \rightarrow \mathbb{Q}_{s}^{n+p}(\tilde{c})$ is an isometric immersion and $\tilde{f}: \tilde{M}^{n} \rightarrow \mathbb{Q}_{s}^{n+p}(\tilde{c})$ is an L-transform of $f$, then $\tilde{M}^{n}$ also has constant curvature $c$.

Proof: We get from (31) that $\Psi$ defined by (27) satisfies $\Psi(Y)=\Phi(Y) \Omega^{-1}(\rho / 2-\Omega L)$, and hence $Q$ given by (28) reduces to $Q(X, Y)=\Phi(X) \Omega^{-1}\left(\rho / 2-L^{t} \Omega^{t}\right)\left(\Omega^{-1}\right)^{t} \Phi(Y)^{t}$. Using (32), we obtain $\hat{Q}(X, Y)=0$, and the statement follows from Corollary 10,

To prove the existence of $L$-transforms we first write (5) and (31) in the local coordinates given by Propositions 1 and 2 .

Lemma 14. Let $f: M^{n}(c) \rightarrow \mathbb{Q}_{s}^{n+p}(c)$ be an isometric immersion satisfying the assumptions of Proposition 1, and let $u_{1}, \ldots, u_{n}, \xi_{1}, \ldots, \xi_{p}$ and $(v, h)$ be, respectively, principal coordinates on an open subset $U \subset M^{n}(c)$, the orthonormal frame of $N_{f} U$ and the pair associated to $f$ given by that result. Given $\varphi: U \rightarrow V$ and $\beta \in \Gamma\left(V^{*} \otimes N_{f} U\right)$, where $V$ is a Euclidean vector space, define $\gamma_{1}, \ldots, \gamma_{n}, \beta^{1}, \ldots, \beta^{p}: U \rightarrow V$ by

$$
\gamma_{i}=v_{i}^{-1} \omega\left(\partial_{i}\right) \text { and } \beta^{r}=\beta^{t}\left(\xi_{r}\right)
$$

for $1 \leq i \leq n$ and $1 \leq r \leq p$, where $\omega=d \varphi$. Then $(\omega, \beta)$ satisfies (5) and $\Phi(\omega, \beta)$ satisfies (31) if and only if $\left(\varphi, \gamma_{1}, \ldots, \gamma_{n}, \beta^{1}, \ldots, \beta^{p}\right)$ is a solution of the linear system of PDE's

$$
\mathcal{R}=\left\{\begin{array}{l}
\left.i) \partial_{i} \varphi=v_{i} \gamma_{i}, \quad i i\right) \partial_{i} \gamma_{j}=h_{j i} \gamma_{i}, \quad i \neq j \\
i i i) \partial_{i} \gamma_{i}=-\sum_{j \neq i} h_{j i} \gamma_{j}-\left(\left(L^{t}\right)^{-1}-I\right) \beta^{i}-c v_{i} \varphi \\
\left.i v) \partial_{i} \beta^{r}=h_{i r} \beta^{i}, i \neq r, \quad v\right) \epsilon_{i} \partial_{i} \beta^{i}=-\gamma_{i}-\sum_{r \neq i} \epsilon_{r} h_{i r} \beta^{r} .
\end{array}\right.
$$


Proof: Equation $(i)$ of $\mathcal{R}$ is equivalent to $\omega=d \varphi$. Now we have

$$
\omega^{t}(v)=\sum_{i=1}^{n} v_{i}^{-1}\left\langle\gamma_{i}, v\right\rangle \partial_{i} \text { and } \beta(v)=\sum_{r=1}^{p} \epsilon_{r}\left\langle\beta^{r}, v\right\rangle \xi_{r},
$$

hence using (11) we obtain $\alpha_{f}\left(\partial_{i}, \omega^{t}(v)\right)=\left\langle\gamma_{i}, v\right\rangle \xi_{i}$ and

$$
\left(\nabla_{\partial_{i}} \beta\right)(v)=\sum_{r=1, r \neq i}^{p} \epsilon_{r}\left\langle\partial_{i} \beta^{r}-h_{i r} \beta^{i}, v\right\rangle \xi_{r}+\left\langle\epsilon_{i} \partial_{i} \beta^{i}+\sum_{r=1, r \neq i}^{p} \epsilon_{r} h_{i r} \beta^{r}, v\right\rangle \xi_{i} .
$$

Thus (5) is equivalent to $(i v)$ and $(v)$. Moreover, using the third formula in (1), it follows that $\Phi=\Phi(\omega, \beta)$ satisfies

$$
\Phi_{v}\left(\partial_{i}\right)=\sum_{j \neq i}\left\langle\partial_{i} \gamma_{j}-h_{j i} \gamma_{i}, v\right\rangle X_{j}+\left\langle\partial_{i} \gamma_{i}+\sum_{j \neq i} \gamma_{j} h_{j i}-\beta^{i}+c v_{i} \varphi, v\right\rangle X_{i},
$$

hence (31) reduces to (ii) and (iii).

Remark 15. Lemma 14 also holds for an isometric immersion $f: M^{n}(c) \rightarrow \mathbb{Q}_{s}^{n+p}(\tilde{c})$, with $c \neq \tilde{c}$, satisfying the assumptions of Proposition 2, if system $\mathcal{R}$ is replaced by

$$
\mathcal{R}^{*}=\left\{\begin{array}{l}
\text { i) } \partial_{i} \varphi=v_{i} \gamma_{i}, \quad \text { ii) } \partial_{i} \gamma_{j}=h_{j i} \gamma_{i}, i \neq j \\
\text { iii) } \partial_{i} \gamma_{i}=-\sum_{j \neq i} h_{j i} \gamma_{j}-\left(\left(L^{t}\right)^{-1}-I\right) \sum_{r} \epsilon_{r} V_{i r} \beta^{r}-\left(\left(L^{t}\right)^{-1}(c-\tilde{c})+\tilde{c}\right) v_{i} \varphi, \\
i v) \partial_{i} \beta^{r}=-V_{i r} \gamma_{i}, \quad i \neq r .
\end{array}\right.
$$

Definition 16. Let $V, W_{1}, W_{2}$ be Euclidean vector spaces. Given $A \in V^{*} \otimes V, c, \tilde{c} \in \mathbb{R}$, $\psi \in V^{*}, \nu \in V^{*} \otimes W_{1}$ and $\beta \in V^{*} \otimes W_{2}$, we say that $(\psi, \nu, \beta)$ is $A$-admissible if the system

$$
\left\{\begin{array}{l}
X+X^{t}=\nu^{t} \nu+\beta^{t} \beta+\tilde{c} \psi^{t} \psi \\
X A+A^{t} X^{t}=\beta^{t} \beta-(c-\tilde{c}) \psi^{t} \psi
\end{array}\right.
$$

admits a unique solution $X \in V^{*} \otimes V$, and if such solution is invertible.

Remark 17. Notice that if the inner product $\langle\cdot, \cdot\rangle$ is replaced by $\langle\cdot, \cdot\rangle^{\sim}$, given by $\langle\cdot, \cdot\rangle^{\sim}=$ $\langle\cdot, B \cdot\rangle$ for some invertible $B \in V^{*} \otimes V$, then $(\psi, \nu, \beta)$ is $A$-admissible if and only if $(\hat{\psi}, \hat{\nu}, \hat{\beta})$, given by $\hat{\psi}=\psi B, \hat{\nu}=\nu B$ and $\hat{\beta}=\beta B$, is $\hat{A}$-admissible, where $\hat{A}=B^{-1} A B$. Namely, system (35) has a unique solution $X$ if and only if the corresponding system for $(\hat{\psi}, \hat{\nu}, \hat{\beta}, \hat{A})$ has a unique solution $\hat{X}$, and these are related by $\hat{X}=X B$. 
Theorem 18. Let $f: M^{n}(c) \rightarrow \mathbb{Q}^{n+p}(\tilde{c})$ be an isometric immersion as in either of Propositions 1 or 2, depending on whether $c=\tilde{c}$ or $c \neq \tilde{c}$, respectively. Given $x_{0} \in M^{n}(c)$, if $L$ is an invertible endomorphism of a Euclidean vector space $V$ and $\left(\psi_{0}, \nu_{0}, \beta_{0}\right)$ is an $L$-admissible triple, with $\nu_{0} \in V^{*} \otimes T_{x_{0}} M$ and $\beta_{0} \in V^{*} \otimes N_{f} M\left(x_{0}\right)$, then there exist an open neighborhood $W$ of $x_{0}$ and a unique L-transform $\tilde{f}=\mathcal{R}_{\varphi, \beta, \Omega, L}\left(\left.f\right|_{W}\right)$ of $\left.f\right|_{W}$ such that $\varphi\left(x_{0}\right)=\psi_{0}^{t}, \omega\left(x_{0}\right)=\nu_{0}^{t}$ and $\beta\left(x_{0}\right)=\beta_{0}$.

Proof: Let $\varphi^{0}, \gamma_{1}^{0}, \ldots, \gamma_{n}^{0}, \beta_{0}^{1}, \ldots, \beta_{0}^{p} \in V$ be given by $\varphi^{0}=\psi_{0}^{t}, \gamma_{i}^{0}=v_{i}^{-1} \nu_{0}\left(\partial_{i}\right)$ and $\beta_{0}^{r}=$ $\beta_{0}^{t}\left(\xi_{r}\right)$. It is immediate to verify, using the fact that $(v, h)$ (resp., $\left.(v, h, V)\right)$ is a solution of (2) (resp., (3) ), that the compatibility conditions of system $\mathcal{R}$ (resp., $\mathcal{R}^{*}$ ) are satisfied. Thus there exists a solution $\left(\varphi, \gamma_{1}, \ldots, \gamma_{n}, \beta^{1}, \ldots, \beta^{p}\right)$ of $\mathcal{R}$ (resp., $\left.\mathcal{R}^{*}\right)$ such that $\varphi\left(x_{0}\right)=\varphi^{0}$, $\gamma_{i}\left(x_{0}\right)=\gamma_{i}^{0}$ and $\beta^{r}\left(x_{0}\right)=\beta_{0}^{r}$ for $1 \leq i \leq n$ and $1 \leq r \leq p$. It follows from Lemma 14 that $\omega=d \varphi$ and $\beta$ given by (34) satisfy (5), (31) and the initial conditions

$$
\varphi\left(x_{0}\right)=\psi_{0}^{t}, \beta\left(x_{0}\right)=\beta_{0} \text { and } \omega\left(x_{0}\right)=\nu_{0}^{t} .
$$

Since the triple $\left(\psi_{0}, \nu_{0}, \beta_{0}\right)$ is $L$-admissible, the system of equations

$$
\left\{\begin{array}{l}
X+X^{t}=\nu_{0}^{t} \nu_{0}+\beta_{0}^{t} \beta_{0}+\tilde{c} \psi_{0}^{t} \psi_{0} \\
X L+L^{t} X^{t}=\beta_{0}^{t} \beta_{0}-(c-\tilde{c}) \psi_{0}^{t} \psi_{0}
\end{array}\right.
$$

has a unique solution $X=\Omega_{0}$, which is invertible. Let $\Omega \in \Gamma\left(V^{*} \otimes V\right)$ be the unique solution of (15) with $\Omega\left(x_{0}\right)=\Omega_{0}$ on an open simply connected neighborhood $W$ of $x_{0}$ where $\Omega$ remains invertible. Using (5) and (17) we obtain

$$
\begin{aligned}
d\left(\Omega L+L^{t} \Omega^{t}-\rho\right)(X)= & \omega \Phi(X) L+L^{t} \Phi(X)^{t} \omega^{t}-d \beta^{t}(X) \beta-\beta^{t} d \beta(X) \\
& +(c-\tilde{c}) \omega(X) \varphi^{t}+(c-\tilde{c}) \varphi \omega(X)^{t} \\
= & \omega\left(\Phi(X) L+A(X) \beta+(c-\tilde{c}) X \varphi^{t}\right) \\
& +\left(\omega\left(\Phi(X) L+A(X) \beta+(c-\tilde{c}) X \varphi^{t}\right)\right)^{t},
\end{aligned}
$$

and the right-hand-side vanishes by (31). Thus (32) holds on $W$ since it holds at $x_{0}$.

It follows that $(\varphi, \beta, \Omega)$ is the unique triple that satisfies the conditions of Definitions 3 and 11 on $W$, as well as the given initial conditions at $x_{0}$. Therefore $\tilde{f}=\mathcal{R}_{\varphi, \beta, \Omega}\left(\left.f\right|_{W}\right)$ is an $L$-transform of $\left.f\right|_{W}$, and it is the unique one defined on an open neighborhood of $x_{0}$ which satisfies the given initial conditions at $x_{0}$.

It remains to investigate when $(\psi, \nu, \beta)$ is an $L$-admissible triple for a given endomorphism $L$, and in particular for which endomorphisms $L$ there exist $L$-admissible triples. First, in the next result we give conditions on $L$ and $(\psi, \nu, \beta)$ under which system (35) has exactly one solution. 
An operator $A \in V^{*} \otimes V$ is said to be nonderogatory if its minimal and characteristic polynomials coincide, or equivalently, if for each eigenvalue $\alpha$ of $A$ the eigenspace $\operatorname{ker}\left(A^{c}-\right.$ $\alpha I)$ of the complexified endomorphism $A^{c} \in\left(V^{c}\right)^{*} \otimes V^{c}, V^{c}=V \otimes \mathbb{C}$, has dimension one. If $a_{11}, \ldots, a_{1 n_{1}}, \ldots, a_{p 1}, \ldots, a_{p n_{p}}$ is the Jordan basis of $A^{c}$, with $a_{i 1}, \ldots, a_{i n_{i}}$ corresponding to the eigenvalue $\alpha_{i}$, we call $a_{i 1}, \ldots, a_{i n_{i}}$ the generalized eigenvectors associated to $\alpha_{i}$. Thus, for $1 \leq i \leq p$ and $1 \leq k_{i} \leq n_{i}$ we have $\left(A^{c}-\alpha_{i} I\right) a_{i k_{i}}=a_{i, k_{i}-1}, \quad a_{i 0}=0$.

Proposition 19. Let $A \in V^{*} \otimes V$ be nonderogatory, let $\alpha_{1}, \ldots, \alpha_{p}$ (resp., $\left.\gamma_{1}, \ldots, \gamma_{q}\right)$ be the real (resp., complex) eigenvalues of $A$, let $a_{i 1}, a_{i 2}, \ldots, a_{i n_{i}}\left(r e s p ., w_{j 1}, w_{j 2}, \ldots, w_{j m_{j}}\right)$ be the generalized eigenvectors of $A$ associated with $\alpha_{i}, 1 \leq i \leq p$ (resp., $\left.\gamma_{j}, 1 \leq j \leq q\right)$. Then system (35) has a solution $X \in V^{*} \otimes V$ if and only if

$$
\sum_{r=0}^{k_{i}-1}\left\langle a_{i, k_{i}-r}, B a_{i, r+1}\right\rangle=0, i=1, \ldots, p \text { and } k_{i}=1,2, \ldots, n_{i}
$$

and

$$
\sum_{r=0}^{\ell_{j}-1}\left\langle w_{j, \ell_{j}-r}, B^{c} \bar{w}_{j, r+1}\right\rangle=0, \quad j=1, \ldots, q \text { and } \ell_{j}=1,2, \ldots, m_{j}
$$

where

$$
B=\left(I-A^{t}\right) \beta^{t} \beta-A^{t} \nu^{t} \nu-\left((c-\tilde{c}) I+\tilde{c} A^{t}\right) \psi^{t} \psi,
$$

$B^{c}$ is its complexification and $\langle\rangle:, V^{c} \times V^{c} \rightarrow \mathbb{C}$ is the canonical hermitian inner product on $V^{c}=V \otimes \mathbb{C}$. Moreover, if (36) and (37) are satisfied then the solution $X$ is unique.

Proof: Let $X_{s}=\frac{1}{2}\left(X+X^{t}\right)$ and $X_{a}=\frac{1}{2}\left(X-X^{t}\right)$ be the symmetric and anti-symmetric parts of $X \in V^{*} \otimes V$, respectively. Then $X$ is a solution of (35) if and only if

$$
2 X_{s}=\nu^{t} \nu+\beta^{t} \beta+\tilde{c} \psi^{t} \psi
$$

and

$$
X_{a} A-A^{t} X_{a}=B_{s}=\left(B+B^{t}\right) / 2 .
$$

Let $\Psi: \mathcal{A}(V) \rightarrow \mathcal{S}(V)$ be the linear map from the space of anti-symmetric endomorphisms of $V$ into the space of symmetric endomorphisms of $V$ given by

$$
\Psi(Y)=Y A-A^{t} Y
$$

A necessary and sufficient condition for $\Psi$ to be injective is that $A$ be a nonderogatory endomorphism (see [14]). Therefore, a solution $X$ of (35) exists if and only if $B_{s}$ belongs to the range of $\Psi$, and in this case $X$ is uniquely determined by $X=X_{s}+X_{a}$, with $X_{s}$ given by (38) and $X_{a}$ the unique element of $\mathcal{A}(V)$ such that $\Psi\left(X_{a}\right)=B_{s}$.

Now, $B_{s}$ belongs to the range of $\Psi$ if and only if its complexification $B_{s}^{c}$ belongs to the range of $\Psi$ regarded as a linear map from $\mathcal{A}\left(V^{c}\right)$ to $\mathcal{S}\left(V^{c}\right)$, where $V^{c}$ is endowed with 
the canonical hermitian inner product and $\mathcal{S}\left(V^{c}\right), \mathcal{A}\left(V^{c}\right)$ denote the spaces of self-adjoint and anti-self-adjoint endomorphisms of $V^{c}$, respectively. This is the case if and only if $B_{s}$, and hence $B$, belongs to the orthogonal complement of the kernel of the adjoint $\Psi^{*}$ of $\Psi$ with respect to the inner product on $V^{c} \otimes V^{c}$ given by

$$
\langle X, Y\rangle=\operatorname{trace} Y^{*} X
$$

where $Y^{*}$ stands for the adjoint of $Y$. It is easily checked that

$$
\Psi^{*} Y=Y A^{t}-A Y
$$

and that a basis of $\operatorname{ker} \Psi^{*}$ is $\left\{Y_{i k_{i}}, Z_{j \ell_{j}}, \bar{Z}_{j \ell_{j}}, 1 \leq k_{i} \leq n_{i}, 1 \leq \ell_{j} \leq m_{j}\right\}$, where

$$
Y_{i k_{i}}=a_{i k_{i}} a_{i 1}^{t}+a_{i, k_{i}-1} a_{i 2}^{t}+\ldots, a_{i 1} a_{i k_{i}}^{t}, \quad 1 \leq i \leq p, \text { and } 1 \leq k_{i} \leq n_{i},
$$

and

$$
Z_{j \ell_{j}}=w_{j \ell_{j}} w_{j 1}^{t}+w_{j, \ell_{j}-1} w_{j 2}^{t}+\ldots, w_{j 1} w_{j \ell_{j}}^{t}, 1 \leq j \leq q \quad \text { and } 1 \leq \ell_{j} \leq m_{j} .
$$

(cf. [9]). Therefore $B_{s}^{c} \in \Psi\left(\mathcal{A}\left(V^{c}\right)\right)$ if and only if $\left\langle B^{c}, Y_{i k_{i}}\right\rangle=0=\left\langle B^{c}, Z_{j \ell_{j}}\right\rangle$ for all $1 \leq i \leq p, 1 \leq k_{i} \leq n_{i}, 1 \leq j \leq q, 1 \leq \ell_{j} \leq m_{j}$, which are equivalent to (36) and (37).

Remark 20. If (36) and (37) are satisfied then the unique solution $X$ of (35) is $X=$ $X_{s}+X_{a}$, where $X_{s}$ is given by (38) and $X_{a}$ is the unique solution of (39), an explicit expression of which can be found in [11].

Next, for $A$ and $(\psi, \nu, \beta)$ as in the preceding proposition, we give further sufficient conditions for the unique solution $X$ of (35) to be invertible, that is, for $(\psi, \nu, \beta)$ to be $A$-admissible, under the assumption that $(c, \tilde{c})$ belongs to the subset

$$
D(c, \tilde{c})=\mathbb{R}^{2} \backslash\{(c, \tilde{c}): \tilde{c}<0 \text { and } c \in[\tilde{c}, 0]\} .
$$

Proposition 21. Let $A \in V^{*} \otimes V$ be nonderogatory and let $(\psi, \nu, \beta)$ satisfy conditions (36) and (37). Set

$$
S=\left\{\begin{array}{l}
\operatorname{ker} \nu \cap \operatorname{ker} \beta, \text { if }(c, \tilde{c})=(0,0) \\
\operatorname{ker} \psi \cap \operatorname{ker} \nu \cap \operatorname{ker} \beta, \text { if }(c, \tilde{c}) \neq(0,0)
\end{array}\right.
$$

and $S^{c}=S \otimes \mathbb{C}$. If $(c, \tilde{c}) \in D(c, \tilde{c})$, then the unique solution $X \in V^{*} \otimes V$ of (35) satisfies

$$
\operatorname{ker} X=\operatorname{ker} X^{t} \subset S \text { and } A(\operatorname{ker} X) \subset \operatorname{ker} X \text {. }
$$

In particular, if

$$
E_{\alpha_{i}} \cap S=\{0\}=E_{\gamma_{j}} \cap S^{c}
$$

for all $1 \leq i \leq p$ and $1 \leq j \leq q$ then $X$ is invertible, and hence $(\psi, \nu, \beta)$ is $A$-admissible. 
Proof: Subtracting the second equation from the first one in (35), we obtain

$$
X(I-A)+\left(I-A^{t}\right) X^{t}=\nu^{t} \nu+c \psi^{t} \psi
$$

Therefore, for all $u \in \operatorname{ker} X^{t}$ we have

$$
0=|\beta u|^{2}+|\nu u|^{2}+\tilde{c} \psi^{2}(u)=|\beta u|^{2}-(c-\tilde{c}) \psi^{2}(u)=|\nu u|^{2}+c \psi^{2}(u) .
$$

For $(c, \tilde{c}) \in D(c, \tilde{c})$, this implies that $\operatorname{ker} X^{t} \subset S$. We obtain from (35) that ker $X=\operatorname{ker} X^{t}$ and $A(\operatorname{ker} X) \subset \operatorname{ker} X$.

The following proposition provides conditions on a nonderogatory endomorphism $A$ for an A-admissible triple $(\psi, \nu, \beta)$ to exist.

Proposition 22. Let $A \in V^{*} \otimes V$ be nonderogatory. If $(c, \tilde{c}) \in D(c, \tilde{c}) \backslash\{(0,0)\}$, then there exists an $A$-admissible triple $(\psi, \nu, \beta)$ if and only if all real eigenvalues of $A$ with odd algebraic multiplicity belong to the subset $Z(c, \tilde{c})$ defined as either $[\kappa, 1],[0,1],[0, \kappa]$, $(-\infty, \kappa] \cup[0, \infty),(-\infty, 1] \cup[\kappa, \infty),(-\infty, 1]$ or $[0, \infty)$, depending on whether $c \geq \tilde{c}>0$, $0 \leq c<\tilde{c}, c<0<\tilde{c}, c<\tilde{c}<0, \tilde{c}<0<c, \tilde{c}=c<c$ or $c<0=\tilde{c}$, respectively, with $\kappa=(\tilde{c}-c) / \tilde{c}$. If $(c, \tilde{c})=(0,0)$ or $(c, \tilde{c}) \notin D(c, \tilde{c})$, such a triple always exists.

Proof: Let $\alpha_{1}, \ldots, \alpha_{p}$ (resp., $\gamma_{1}, \ldots, \gamma_{q}$ ) be the real (resp., complex) eigenvalues of the nonderogatory endomorphism $A$, and $a_{i 1}, a_{i 2}, \ldots, a_{i n_{i}}$ (resp., $w_{j 1}, w_{j 2}, \ldots, w_{j m_{j}}$ ) the generalized eigenvectors of $A$ associated with $\alpha_{i}, 1 \leq i \leq p$ (resp., $\gamma_{j}, 1 \leq j \leq q$ ). We denote by $R_{w_{j l}}$ and $I_{w_{j l}}$ the real and imaginary parts of $w_{j l}$, respectively. We will show that, if $(c, \tilde{c}) \in D(c, \tilde{c}) \backslash\{(0,0)\}$, then one can choose $y_{i k_{i}}, y_{j \ell_{j}}^{R}, y_{j \ell_{j}}^{I} \in \mathbb{R}, Y_{i k_{i}}, Y_{j \ell_{j}}^{R}, Y_{j \ell_{j}}^{I} \in W_{1}$ and $\xi_{i k_{i}}, \xi_{j \ell_{j}}^{R}, \xi_{j \ell_{j}}^{I} \in W_{2}, 1 \leq k_{i} \leq n_{i}, 1 \leq \ell_{j} \leq m_{j}$, so that the triple $(\psi, \nu, \beta)$ defined by $\psi\left(a_{i k_{i}}\right)=y_{i k_{i}}, \psi\left(R_{w_{j \ell_{j}}}\right)=y_{j \ell_{j}}^{R}, \psi\left(I_{w_{j \ell_{j}}}\right)=y_{j \ell_{j}}^{I}, \nu\left(a_{i k_{i}}\right)=Y_{i k_{i}}, \nu\left(R_{w_{j \ell_{j}}}\right)=Y_{j \ell_{j}}^{R}$, $\nu\left(I_{w_{j \ell_{j}}}\right)=Y_{j \ell_{j}}^{I}, \beta\left(a_{i k_{i}}\right)=\xi_{i k_{i}}, \beta\left(R_{w_{j \ell_{j}}}\right)=\xi_{j \ell_{j}}^{R}$ and $\beta\left(I_{w_{j \ell_{j}}}\right)=\xi_{j \ell_{j}}^{I}$ is $A$-admissible if and only if $A$ satisfies the conditions in the statement. If $(c, \tilde{c})=(0,0)$ or $(c, \tilde{c}) \notin D(c, \tilde{c})$, we will show that such a choice is always possible.

Claim 23. One can choose $y_{j \ell_{j}}^{R}, y_{j \ell_{j}}^{I} \in \mathbb{R}, Y_{j \ell_{j}}^{R}, Y_{j \ell_{j}}^{I} \in W_{1}$ and $\xi_{j \ell_{j}}^{R}, \xi_{j \ell_{j}}^{I} \in W_{2}, 1 \leq j \leq q$, $1 \leq \ell_{j} \leq m_{j}$, so that (37) is satisfied and either $\left(y_{j 1}^{R}, Y_{j 1}^{R}, \xi_{j 1}^{R}\right)$ or $\left(y_{j 1}^{I}, Y_{j 1}^{I}, \xi_{j 1}^{I}\right)$ is nontrivial.

Proof: For $\ell_{j}=1$, denoting by $R_{\gamma_{j}}$ and $I_{\gamma_{j}}$ the real and imaginary parts of $\gamma_{j}$, respectively, equation (37) becomes

$$
\begin{aligned}
& \left(1-R_{\gamma_{j}}\right)\left(\left|\xi_{j 1}^{R}\right|^{2}-\left|\xi_{j 1}^{I}\right|^{2}\right)+2 I_{\gamma_{j}}\left\langle\xi_{j 1}^{R}, \xi_{j 1}^{I}\right\rangle-R_{\gamma_{j}}\left(\left|Y_{j 1}^{R}\right|^{2}-\left|Y_{j 1}^{I}\right|^{2}\right) \\
& \left.\quad+2 I_{\gamma_{j}}\left\langle Y_{j 1}^{R}, Y_{j 1}^{I}\right\rangle+\left((c-\tilde{c})+R_{\gamma_{j}} \tilde{c}\right)\left(\left(y_{j 1}^{I}\right)^{2}-\left(y_{j 1}^{R}\right)^{2}\right)\right)+2 \tilde{c} I_{\gamma_{j}} y_{j 1}^{R} y_{j 1}^{I}=0
\end{aligned}
$$


and

$$
\begin{aligned}
& -I_{\gamma_{j}}\left(\left|\xi_{j 1}^{R}\right|^{2}-\left|\xi_{j 1}^{I}\right|^{2}\right)+2\left(1-R_{\gamma_{j}}\right)\left\langle\xi_{j 1}^{R}, \xi_{j 1}^{I}\right\rangle-I_{\gamma_{j}}\left(\left|Y_{j 1}^{R}\right|^{2}-\left|Y_{j 1}^{I}\right|^{2}\right) \\
& \quad-2 R_{\gamma_{j}}\left\langle Y_{j 1}^{R}, Y_{j 1}^{I}\right\rangle+\tilde{c} I_{\gamma_{j}}\left(\left(y_{j 1}^{I}\right)^{2}-\left(y_{j 1}^{R}\right)^{2}\right)+2\left((c-\tilde{c})+\tilde{c} R_{\gamma_{j}}\right) y_{j 1}^{R} y_{j 1}^{I}=0 .
\end{aligned}
$$

It is easy to see that, for each $1 \leq j \leq q$, one can choose a solution $\left(\xi_{j 1}^{R}, \xi_{j 1}^{I}, Y_{j 1}^{R}, Y_{j 1}^{I}, y_{j 1}^{R}, y_{j 1}^{I}\right)$ of both of the preceding equations so that $\left(y_{j 1}^{R}, Y_{j 1}^{R}, \xi_{j 1}^{R}\right) \neq(0,0,0)$ or $\left(y_{j 1}^{I}, Y_{j 1}^{I}, \xi_{j 1}^{I}\right) \neq$ $(0,0,0)$. Once $\xi_{j \ell_{j}}^{R}, \xi_{j \ell_{j}}^{I}, Y_{j \ell_{j}}^{R}, Y_{j \ell_{j}}^{I}, y_{j \ell_{j}}^{R}, y_{j \ell_{j}}^{I}$ have been chosen for $1 \leq \ell_{j} \leq k_{j}-1 \leq m_{j}-1$, then (37) for $\ell_{j}=k_{j}$ becomes a pair of linear equations on $\left(\xi_{j k_{j}}^{R}, \xi_{j k_{j}}^{I}, Y_{j k_{j}}^{R}, Y_{j k_{j}}^{I}, y_{j k_{j}}^{R}, y_{j k_{j}}^{I}\right)$, which are easily checked to admit a common solution.

Claim 24. If $(c, \tilde{c}) \in D(c, \tilde{c}) \backslash\{(0,0)\}$ and $\alpha_{i} \in Z(c, \tilde{c})$ then one can choose $y_{i k_{i}}, Y_{i k_{i}}, \xi_{i k_{i}}$, $1 \leq i \leq p, 1 \leq k_{i} \leq n_{i}$, such that (36) is satisfied and $\left(y_{i 1}, Y_{i 1}, \xi_{i 1}\right) \neq(0,0,0)$. The same statement is true if $(c, \tilde{c})=(0,0)$.

Proof: Given $1 \leq i \leq p$, equation (36) for $k_{i}=1$ becomes

$$
\left(1-\alpha_{i}\right)\left|\xi_{i 1}\right|^{2}-\alpha_{i}\left|Y_{i 1}\right|^{2}-\left((c-\tilde{c})+\alpha_{i} \tilde{c}\right)\left(y_{i 1}\right)^{2}=0 .
$$

One can choose $\left(y_{i 1}, Y_{i 1}, \xi_{i 1}\right) \neq(0,0,0)$ satisfying (43) if the coefficients $\left(1-\alpha_{i}\right),-\alpha_{i}$ and $-\left((c-\tilde{c})+\alpha_{i} \tilde{c}\right)$ are neither all positive nor all negative, which is easily seen to be the case if $(c, \tilde{c}) \in D(c, \tilde{c}) \backslash\{(0,0)\}$ and $\alpha_{i} \in Z(c, \tilde{c})$. Once $\left(y_{i 1}, Y_{i 1}, \xi_{i 1}\right) \neq(0,0,0)$ satisfying (43) has been chosen, it is easy to check that $y_{i k_{i}}, Y_{i k_{i}}, \xi_{i k_{i}}, 2 \leq k_{i} \leq n_{i}$, can be chosen so that the remaining equations in (36) are satisfied.

Claim 25. Suppose that $(c, \tilde{c}) \in D(c, \tilde{c}) \backslash\{(0,0)\}$ and $\alpha_{i} \notin Z(c, \tilde{c})$ for some $i \in\{1, \ldots, p\}$. If $\alpha_{i}$ has even algebraic multiplicity then $y_{i l_{i}}=Y_{i l_{i}}=\xi_{i l_{i}}=0$ for $l_{i} \leq n_{i} / 2$, and one can choose $y_{i l_{i}}, Y_{i l_{i}}, \xi_{i l_{i}}, n_{i} / 2+1 \leq l_{i} \leq n_{i}$, so that $a_{i 1} \notin \operatorname{ker} X$, where $X$ is the unique solution of (35).

Proof: Since $\alpha_{i} \notin Z(c, \tilde{c})$, the numbers $\left(1-\alpha_{i}\right),-\alpha_{i},-\left((c-\tilde{c})+\alpha_{i} \tilde{c}\right)$ are either all positive or all negative. Since $n_{i}$ is even, it is easy to check that a solution $\left(y_{i l_{i}}, Y_{i l_{i}}, \xi_{i l_{i}}\right)$ of (36) ) must satisfy $y_{i l_{i}}=Y_{i l_{i}}=\xi_{i l_{i}}=0$ for $l_{i} \leq n_{i} / 2$, and that $y_{i l_{i}}, Y_{i l_{i}}$ and $\xi_{i l_{i}}$ may be arbitrarily chosen for $l_{i}>n_{i} / 2$. We choose a solution such that $\left(y_{i l_{i}}, Y_{i l_{i}}, \xi_{i l_{i}}\right) \neq(0,0,0)$ for $l_{i}=n_{i} / 2+1$. Using that $A$ is nonderogatory, we obtain that the unique solution $X$ of (35) satisfies $\left\langle X a_{i 1}, a_{i l_{i}}\right\rangle=\left\langle X a_{i 1}, a_{j k_{j}}\right\rangle=\left\langle X a_{i 1}, R_{w_{j \ell_{j}}}\right\rangle=\left\langle X a_{i 1}, I_{w_{j \ell_{j}}}\right\rangle=0$ for $1 \leq l_{i} \leq n_{i}-1,1 \leq k_{j} \leq n_{j}$ and $1 \leq \ell_{j} \leq m_{j}$, hence

$$
X\left(a_{i 1}\right) \in \operatorname{span}\left\{a_{i n_{i}}\right\} .
$$


Moreover,

$$
\begin{aligned}
0= & \left\langle\left(X_{a} A-A^{t} X_{a}-B_{s}\right) a_{i l_{i}}, a_{i k_{i}}\right\rangle=\left\langle X_{a} a_{i, l_{i}-1}, a_{i k_{i}}\right\rangle-\left\langle X_{a} a_{i l_{i}}, a_{i, k_{i}-1}\right\rangle \\
& -\left\langle B_{s} a_{i l_{i}}, a_{i k_{i}}\right\rangle, 1 \leq l_{i}, k_{i} \leq n_{i} .
\end{aligned}
$$

Using that $B_{s}\left(a_{i l_{i}}\right)=0$ for $l_{i} \leq n_{i} / 2$, we obtain from the preceding relations that

$$
\left\langle X a_{i 1}, a_{i n_{i}}\right\rangle=\left\langle X_{a} a_{i 1}, a_{i n_{i}}\right\rangle=\left\langle X_{a} a_{i, \frac{n_{i}}{2}}, a_{i, \frac{n_{i}}{2}+1}\right\rangle=\left\langle B_{s} a_{i, \frac{n_{i}}{2}+1}, a_{i, \frac{n_{i}}{2}+1}\right\rangle / 2,
$$

Since $\left(y_{i, \frac{n_{i}}{2}+1}, Y_{i, \frac{n_{i}}{2}+1}, \xi_{i, \frac{n_{i}}{2}+1}\right) \neq(0,0,0)$ and $\alpha_{i} \notin Z(c, \tilde{c})$, we have

$$
\left\langle B_{s} a_{i, \frac{n_{i}}{2}+1}, a_{i, \frac{n_{i}}{2}+1}\right\rangle=\left(1-\alpha_{i}\right)\left|\xi_{i, \frac{n_{i}}{2}+1}\right|^{2}-\alpha_{i}\left|Y_{i, \frac{n_{i}}{2}+1}\right|^{2}-\left((c-\tilde{c})-\alpha_{i} \tilde{c}\right) y_{i, \frac{n_{i}}{2}+1}^{2} \neq 0 .
$$

Thus $a_{i 1} \notin k e r(X)$.

It follows from Proposition 21 and Claims 23 -25 that, when $(c, \tilde{c}) \in D(c, \tilde{c}) \backslash\{(0,0)\}$, the real eigenvalues of $A$ with odd algebraic multiplicity belonging to $Z(c, \tilde{c})$ is a sufficient condition for an $A$-admissible triple to exist. To complete the proof of Proposition 22 when $(c, \tilde{c}) \in D(c, \tilde{c}) \backslash\{(0,0)\}$, it remains to show that this condition is also necessary. In fact, if $\alpha_{i} \notin Z(c, \tilde{c})$ is such that $n_{i}$ is odd, it is easy to check that a solution $\left(y_{i l_{i}}, Y_{i l_{i}}, \xi_{i l_{i}}\right)$ of (36) must satisfy $y_{i l_{i}}=Y_{i l_{i}}=\xi_{i l_{i}}=0$ for $l_{i} \leq\left(n_{i}+1\right) / 2$, and that $y_{i l_{i}}, Y_{i l_{i}}$ and $\xi_{i l_{i}}$ may be arbitrarily chosen for $l_{i}>\left(n_{i}+1\right) / 2$. If $X$ is the unique solution of (35), using that $B_{s}\left(a_{i l_{i}}\right)=0$ for $l_{i} \leq\left(n_{i}+1\right) / 2$ we obtain from (45) that

$$
\left\langle X_{a} a_{i 1}, a_{i n_{i}}\right\rangle=\left\langle X_{a} a_{i,\left(n_{i}+1\right) / 2}, a_{i,\left(n_{i}+1\right) / 2}\right\rangle=0 .
$$

In view of (44), this implies that $a_{i 1} \in \operatorname{ker} X$.

Now assume that $(c, \tilde{c}) \notin D(c, \tilde{c})$ and suppose that $\operatorname{dim} W_{2} \leq \operatorname{dim} W_{1}$, the argument for the case $\operatorname{dim} W_{1}<\operatorname{dim} W_{2}$ being similar. Choose $\beta \in V^{*} \otimes W_{2}$ so that $R_{w_{j 1}} \notin \operatorname{ker} \beta$, $I_{w_{j 1}} \notin \operatorname{ker} \beta$, and so that $a_{i 1} \in \operatorname{ker} \beta$ only if $\alpha_{i}=\kappa=(\tilde{c}-c) / \tilde{c}$. Let $\nu \in V^{*} \otimes W_{1}$ be given by $\nu=\lambda P \circ \beta$, where $P \in W_{2}^{*} \otimes W_{1}$ satisfies $P^{t} P=I$ and $\lambda \in \mathbb{R} /\{0\}$. Under these conditions, we have $\operatorname{ker} \nu=\operatorname{ker} \beta$ and $\nu^{t} \nu=\lambda^{2} \beta^{t} \beta$.

Again, we will show that one can choose $y_{i k_{i}}, y_{j \ell_{j}}^{R}, y_{j \ell_{j}}^{I} \in \mathbb{R}$ so that the triple $(\psi, \nu, \beta)$, with $\psi$ defined by $\psi\left(a_{i k_{i}}\right)=y_{i k_{i}}, \psi\left(R_{w_{j \ell_{j}}}\right)=y_{j \ell_{j}}^{R}$ and $\psi\left(I_{w_{j \ell_{j}}}\right)=y_{j \ell_{j}}^{I}$, is $A$-admissible.

Denoting $\beta\left(a_{i 1}\right)=\xi_{i 1}$, equation (36) for $k_{i}=1$ becomes

$$
\left(1-\alpha_{i}\left(1+\lambda^{2}\right)\right)\left|\xi_{i 1}\right|^{2}-\left((c-\tilde{c})+\alpha_{i} \tilde{c}\right)\left(y_{i 1}\right)^{2}=0
$$

which always admits a nontrivial solution $\left(y_{i 1}, \xi_{i 1}\right) \neq(0,0)$ if the coefficients $\left(1-\alpha_{i}\left(1+\lambda^{2}\right)\right)$ and $-\left((c-\tilde{c})+\alpha_{i} \tilde{c}\right)$ are both nonzero and have opposite signs, which we can assume to 
be the case by an appropriate choice of $\lambda$. It also admits a nontrivial solution if at least one such coefficient is zero. Notice that when $-\left((c-\tilde{c})+\alpha_{i} \tilde{c}\right)=0$, that is, for $\alpha_{i}=\kappa$, this is due to the fact that we have chosen $a_{i 1} \in \operatorname{ker} \beta$ when $\alpha_{i}=\kappa$.

Denoting $\beta\left(a_{i k_{i}}\right)=\xi_{i k_{i}}, 1 \leq i \leq p$ and $2 \leq k_{i} \leq n_{i}$, it is easy to check that $y_{i k_{i}}$ can be defined so that the remaining equations in (36) are satisfied. For (37), it is sufficient to replace $\nu^{t} \nu=\lambda^{2} \beta^{t} \beta$ in (41) and (42), denoting $\beta\left(R_{w_{j \ell_{j}}}\right)=\xi_{j \ell_{j}}^{R}$ and $\beta\left(I_{w_{j \ell_{j}}}\right)=\xi_{j \ell_{j}}^{I}$ for $1 \leq j \leq q$ and $1 \leq \ell_{j} \leq m_{j}$, and proceed as in the proof of that claim. In this way, one can define $\psi$ in the Jordan basis of $A$ so that (36) and (37) are satisfied and

$$
E_{\alpha_{i}} \cap S=\{0\}=E_{\gamma_{j}} \cap S^{c}
$$

for all $1 \leq j \leq q$ and $1 \leq \ell_{j} \leq m_{j}$, where $S=\operatorname{ker} \beta \cap \operatorname{ker} \psi$ and $S^{c}=S \otimes \mathbb{C}$.

Now, for a solution $X$ of (35), for all $u \in \operatorname{ker} X^{t}$ we have

$$
0=\left(1+\lambda^{2}\right)|\beta u|^{2}+\tilde{c} \psi^{2}(u)=|\beta u|^{2}-(c-\tilde{c}) \psi^{2}(u)=\lambda^{2}|\beta u|^{2}+c \psi^{2}(u) .
$$

Choosing $\lambda$ so that $\lambda^{2}+1 \neq \frac{1}{\kappa}$, the above system only admits the trivial solution $|\beta u|=$ $\psi(u)=0$, and hence $\operatorname{ker} X^{t} \subset S$. Then, we obtain from (35) that $\operatorname{ker} X=\operatorname{ker} X^{t}$ and $A(\operatorname{ker} X) \subset \operatorname{ker} X$. It follows from (46) that $\operatorname{ker} X=\{0\}$, and hence $(\psi, \nu, \beta)$ is $A$-admissible.

In case $A \in V^{*} \otimes V$ is a symmetric endomorphism with $k=\operatorname{dim} V$ distinct eigenvalues (see part (ii) of Remarks 32 below), one has the following more precise statement.

Proposition 26. Let $A \in V^{*} \otimes V$ be symmetric with $k=\operatorname{dim} V$ distinct eigenvalues $\alpha_{1}, \ldots, \alpha_{k}$. Let $a_{1}, \ldots, a_{k}$ be eigenvectors of $A$ associated to $\alpha_{1}, \ldots, \alpha_{k}$, respectively. Then system (35) has a solution if and only if

$$
\left(1-\alpha_{i}\right)\left|\beta\left(a_{i}\right)\right|^{2}-\alpha_{i}\left|\nu\left(a_{i}\right)\right|^{2}-\left((c-\tilde{c})+\alpha_{i} c\right) \psi\left(a_{i}\right)^{2}=0, \quad 1 \leq i \leq k .
$$

If these equations are satisfied, then the solution of (35) is unique and given by

$$
\left\langle X a_{i}, a_{i}\right\rangle=\frac{1}{2} G_{i i} \quad \text { and }\left\langle X a_{i}, a_{j}\right\rangle=\frac{1}{\alpha_{i}-\alpha_{j}}\left(\rho_{i j}-\alpha_{j} G_{i j}\right), \quad i \neq j,
$$

where

$$
\rho_{i j}=\left\langle\beta\left(a_{i}\right), \beta\left(a_{j}\right)\right\rangle-(c-\tilde{c}) \psi\left(a_{i}\right) \psi\left(a_{j}\right)
$$

and

$$
G_{i j}=\left\langle\nu\left(a_{i}\right), \nu\left(a_{j}\right)\right\rangle+\left\langle\beta\left(a_{i}\right), \beta\left(a_{j}\right)\right\rangle+\tilde{c} \psi\left(a_{i}\right) \psi\left(a_{j}\right) .
$$

Moreover, if $(c, \tilde{c}) \in D(c, \tilde{c})$ then $X$ is invertible, that is, $(\psi, \nu, \beta)$ is A-admissible, if and only if $\left(\psi\left(a_{i}\right), \nu\left(a_{i}\right), \beta\left(a_{i}\right)\right) \neq(0,0,0)$ for all $1 \leq i \leq k$. Furthermore, there exists an $A$-admissible triple $(\psi, \nu, \beta)$ if and only if $\alpha_{i} \in Z(c, \tilde{c})$ for all $1 \leq i \leq k$. If $(c, \tilde{c})=(0,0)$ or $(c, \tilde{c}) \notin D(c, \tilde{c})$, an $A$-admissible triple $(\psi, \nu, \beta)$ always exists. 
Proof: For a symmetric endomorphism $A \in V^{*} \otimes V$ with $k$ distinct eigenvalues $\alpha_{1}, \ldots, \alpha_{k}$, it is easily checked that equations (36) reduce to (47). Thus the first assertion follows from Proposition 19, Equations (48) follow directly from system (35), hence they necessarily provide the unique solution of (35).

If $(c, \tilde{c}) \in D(c, \tilde{c})$, that $X$ is invertible if $\left(\psi\left(a_{i}\right), \nu\left(a_{i}\right), \beta\left(a_{i}\right)\right) \neq(0,0,0)$ for all $1 \leq i \leq k$ follows from Proposition 21. For the converse, if $\left(\psi\left(a_{i}\right), \nu\left(a_{i}\right), \beta\left(a_{i}\right)\right)=(0,0,0)$ for some $1 \leq i \leq k$, it follows from the first equation in (35) that $\left\langle X_{s} a_{i}, a_{i}\right\rangle=0$, hence $\left\langle X a_{i}, a_{i}\right\rangle=$ $\left\langle X_{a} a_{i}, a_{i}\right\rangle=0$. On the other hand, the second equation in (35) gives

$$
\left.0=\left\langle\left(X A+A^{t} X^{t}\right)-\beta^{t} \beta-(c-\tilde{c}) \psi^{t} \psi\right) a_{i}, a_{j}\right\rangle=\left(\alpha_{i}-\alpha_{j}\right)\left\langle X a_{i}, a_{j}\right\rangle, \quad i \neq j,
$$

hence $X a_{i}=0$. The two last assertions follow from Proposition 22 ,

Before concluding this section, we compute the pair $(\widetilde{v}, \widetilde{h})$ (resp., the triple $(\widetilde{v}, \widetilde{h}, \widetilde{V})$ ) associated to the $L$-transform of an isometric immersion $f: M^{n}(c) \rightarrow \mathbb{Q}_{s}^{n+p}(c)$ (resp., $\left.f: M^{n}(c) \rightarrow \mathbb{Q}_{s}^{n+p}(\tilde{c}), c \neq \tilde{c}\right)$.

Proposition 27. Let $f: M^{n}(c) \rightarrow \mathbb{Q}_{s}^{n+p}(c)$ be an isometric immersion and $\xi_{1}, \ldots, \xi_{p}$ an orthonormal frame of $N_{f} M$ as in Proposition 1 . Let $(v, h)$ be the pair associated to $f$. If $\tilde{f}=\mathcal{R}_{\varphi, \beta, L}(f)$ is an L-transform of $f$, then $\widetilde{\xi}_{1}, \ldots, \widetilde{\xi}_{p}$ defined by

$$
\widetilde{\xi}_{r}=\mathcal{P}\left(\xi_{r}-\sum_{\ell=1}^{p} \epsilon_{\ell}\left\langle\beta^{r}, L^{-1} \Omega^{-1} \beta^{\ell}\right\rangle \xi_{\ell}\right)
$$

is an orthonormal frame of $N_{\tilde{f}} M$ satisfying the conditions of Proposition 1 , and the pair $(\widetilde{v}, \widetilde{h})$ associated to $\widetilde{f}$ is given by

$$
\widetilde{v}_{j}=v_{j}+\left\langle\beta^{j}, L^{-1} \Omega^{-1} \varphi\right\rangle \text { and } \widetilde{h}_{i r}=h_{i r}+\left\langle\beta^{r}, L^{-1} \Omega^{-1} \gamma_{i}\right\rangle \text {, }
$$

where $\gamma_{1}, \ldots, \gamma_{n}, \beta^{1}, \ldots, \beta^{p}$ are given by (33). In particular, $(\widetilde{v}, \widetilde{h})$ is a new solution of (2).

Proof: By Proposition 6, the metrics $\langle$,$\rangle and \langle,\rangle \sim$ induced by $f$ and $\tilde{f}$, respectively, are related by $\langle,\rangle^{\sim}=D^{*}\langle$,$\rangle , where D=I-\Phi_{\Omega^{-1} \varphi}$ for $\Phi=\Phi(\omega, \beta)$. It follows from (31) that

$$
\Phi_{L v} \partial_{i}=-A_{\beta(v)} \partial_{i}=-\left\langle\beta^{i}, v\right\rangle X_{i}
$$

where $X_{i}=v_{i}^{-1} \partial_{i}$. Thus

$$
D \partial_{i}=\widetilde{v}_{i} X_{i}
$$


and the first of formulas (50) follows. Using (11), (20), (34), (151) and (52), we have for $1 \leq i \leq n$ that

$$
\begin{aligned}
\tilde{\alpha}\left(\partial_{i}, \partial_{i}\right) & =\mathcal{P}\left(\alpha\left(\partial_{i}, D \partial_{i}\right)+\beta\left(\Omega^{-1}\right)^{t} \Phi\left(\partial_{i}\right)^{t} D \partial_{i}\right) \\
& =\mathcal{P}\left(\widetilde{v}_{i} \xi_{i}+\widetilde{v}_{i} \beta\left(\Omega^{-1}\right)^{t} \Phi\left(\partial_{i}\right)^{t} X_{i}\right) \\
& =\tilde{v}_{i} \mathcal{P}\left(\xi_{i}-\sum_{\ell=1}^{p} \epsilon_{\ell}\left\langle\beta^{i}, L^{-1} \Omega^{-1} \beta^{\ell}\right\rangle \xi_{\ell}\right)
\end{aligned}
$$

hence $\tilde{\xi}_{1}, \ldots, \tilde{\xi}_{n}$ are given by (49). On the other hand, from (1), (17), (18) and equations $i v)$ and $(v)$ of system $\mathcal{R}$ in Lemma 14 we obtain

$$
\widetilde{\nabla} \frac{\perp}{\partial_{i}} \widetilde{\xi}_{r}=\left(h_{i r}+\left\langle\beta^{r}, L^{-1} \Omega^{-1} \gamma_{i}\right\rangle\right) \mathcal{P}\left(\xi_{i}-\sum_{\ell=1}^{p} \epsilon_{\ell}\left\langle\beta^{i}, L^{-1} \Omega^{-1} \beta^{\ell}\right\rangle \xi_{\ell}\right),
$$

which shows that $\widetilde{\xi}_{1}, \ldots, \widetilde{\xi}_{p}$ is an orthonormal frame of $N_{\tilde{f}} M$ satisfying the conditions of Proposition 1 and that $\tilde{h}_{i r}$ is given by (50) for $1 \leq i \leq n$ and $1 \leq r \leq p$.

Arguing in a similar way one obtains the following result in the case $c \neq \tilde{c}$.

Proposition 28. Let $f: M^{n}(c) \rightarrow \mathbb{Q}_{s}^{n+p}(\tilde{c}), c \neq \tilde{c}$, be an isometric immersion of a simply connected Riemannian manifold satisfying the assumptions of Proposition 2, let $\xi_{1}, \ldots, \xi_{p}$ be a parallel orthonormal frame of $N_{f} M$ and let $(v, h, V)$ be the triple associated to $f$ and $\xi_{1}, \ldots, \xi_{p}$. If $\tilde{f}=\mathcal{R}_{\varphi, \beta, L}(f)$ is an L-transform of $f$, then $\widetilde{\xi}_{1}=\mathcal{P} \xi_{1}, \ldots, \widetilde{\xi}_{p}=\mathcal{P} \xi_{p}$ is a parallel orthonormal frame of $N_{\tilde{f}} M$, and the triple $(\tilde{v}, \tilde{h}, \tilde{V})$ associated to $\widetilde{f}$ and $\widetilde{\xi}_{1}, \ldots, \widetilde{\xi}_{p}$ is given by

$$
\tilde{v}_{j}=v_{j}-\left\langle B_{j}, \Omega^{-1} \varphi\right\rangle, \tilde{h}_{i j}=h_{i j}-\left\langle B_{j}, \Omega^{-1} \gamma_{i}\right\rangle \text { and } \tilde{V}_{i r}=V_{i r}+\epsilon_{r}\left\langle B_{i}, \Omega^{-1} \beta^{r}\right\rangle,
$$

with $B_{i}=-\left(L^{t}\right)^{-1}\left(\sum_{r=1}^{p} \beta^{r} V_{i r}-(c-\tilde{c}) v_{i} \varphi\right)$. In particular, $(\tilde{v}, \tilde{h}, \tilde{V})$ is a solution of (3).

In the last result of this section we study the inverse of an $L$-transformation. By Proposition 13 in [2], if $\tilde{f}=\mathcal{R}_{\varphi, \beta, \Omega}(f): \tilde{M}^{n} \rightarrow \mathbb{Q}_{s}^{n+p}(\tilde{c})$ is the vectorial Ribaucour transform of $f: M^{n} \rightarrow \mathbb{Q}_{s}^{n+p}(\tilde{c})$ determined by $(\varphi, \beta, \Omega)$ as in Definition 5 , then

$$
\tilde{\varphi}=\Omega^{-1} \varphi, \tilde{\beta}=\mathcal{P} \beta\left(\Omega^{-1}\right)^{t} \text { and } \tilde{\Omega}=\Omega^{-1}
$$

satisfy the conditions of Definition 5 for $\tilde{f}$ and $f=\mathcal{R}_{\tilde{\varphi}, \tilde{\beta}, \tilde{\Omega}}(\tilde{f})$. The next result states what else can be said if $\tilde{f}$ is an $L$-transform of $f$.

Proposition 29. If $\tilde{f}=\mathcal{R}_{\varphi, \beta, \Omega, L}(f)$ is an L-transform, then $f=\mathcal{R}_{\tilde{\varphi}, \tilde{\beta}, \tilde{\Omega}}(\tilde{f})$ is an $L^{t}$ transform of $\tilde{f}$. 
Proof: It follows from (32) that $L\left(\Omega^{t}\right)^{-1}+\Omega^{-1} L^{t}=\Omega^{-1} \rho\left(\Omega^{t}\right)^{-1}$. On the other hand, by Proposition 3 in [2] we have $D \tilde{\Phi}_{v}=-\Phi_{\Omega^{-1} v}$ for all $v \in V$. Hence, using (19) and (31) we obtain

$$
\begin{aligned}
D \tilde{\Phi}(X) L^{t}+ & D A^{\tilde{f}}(X) \tilde{\beta}+(c-\tilde{c}) D X \tilde{\varphi}^{t} \\
= & -\Phi(X) \Omega^{-1} L^{t}+A(X) \beta\left(\Omega^{-1}\right)^{t}+\Phi(X) \Omega^{-1} \beta^{t} \beta\left(\Omega^{-1}\right)^{t}+(c-\tilde{c}) D X \varphi^{t}\left(\Omega^{-1}\right)^{t} \\
= & \Phi(X) L\left(\Omega^{-1}\right)^{t}-\Phi(X) \Omega^{-1} \rho\left(\Omega^{-1}\right)^{t}+A(X) \beta\left(\Omega^{-1}\right)^{t}+\Phi(X) \Omega^{-1} \beta^{t} \beta\left(\Omega^{-1}\right)^{t} \\
& +(c-\tilde{c}) X \varphi^{t}\left(\Omega^{-1}\right)^{t}-(c-\tilde{c}) \Phi(X) \Omega^{-1} \varphi \varphi^{t}\left(\Omega^{-1}\right)^{t}=0
\end{aligned}
$$

Moreover,

$$
\tilde{\Omega} L^{t}+L \tilde{\Omega}^{t}=\Omega^{-1} \beta^{t} \mathcal{P}^{t} \mathcal{P} \beta\left(\Omega^{t}\right)^{-1}-(c-\tilde{c}) \Omega^{-1} \varphi \varphi^{t}\left(\Omega^{-1}\right)^{t}=\tilde{\beta}^{t} \tilde{\beta}-(c-\tilde{c}) \tilde{\varphi} \tilde{\varphi}^{t}
$$

\section{A decomposition theorem for the $L$-transformation}

To prove a decomposition theorem for the $L$-transformation we need the following.

Lemma 30. Under the assumptions of Theorem 7 , the tensor

$$
\bar{\Phi}_{i}(X)=\nabla_{X} \bar{\omega}_{i}^{t}-A^{j}(X) \bar{\beta}_{i}+\tilde{c} X \bar{\varphi}_{i}^{t}
$$

where $\bar{\omega}_{i}=d \bar{\varphi}_{i}$, satisfies

$$
D_{j} \bar{\Phi}_{i}(X)=\Phi_{i}(X)-\Phi_{j}(X) \Omega_{j j}^{-1} \Omega_{j i}, \quad 1 \leq i \neq j \leq 2,
$$

where $D_{j}=I-\Phi_{\Omega_{j j}^{-1} \varphi_{j}}^{j}$.

Proof: We have

$$
\begin{aligned}
\bar{\omega}_{i}(X) & =\omega_{i}(X)-d \Omega_{i j}(X) \Omega_{j j}^{-1} \varphi_{j}+\Omega_{i j} \Omega_{j j}^{-1} d \Omega_{j j}(X) \Omega_{j j}^{-1} \varphi_{j}-\Omega_{i j} \Omega_{j j}^{-1} \omega_{j}(X) \\
& =\omega_{i}(X)-\omega_{i}\left(\Phi^{j}(X) \Omega_{j j}^{-1} \varphi_{j}\right)+\Omega_{i j} \Omega_{j j}^{-1} \omega_{j}\left(\Phi^{j}(X) \Omega_{j j}^{-1} \varphi_{j}\right)-\Omega_{i j} \Omega_{j j}^{-1} \omega_{j}(X) \\
& =\omega_{i}\left(D_{j} X\right)-\Omega_{i j} \Omega_{j j}^{-1} \omega_{j}\left(D_{j} X\right) .
\end{aligned}
$$

Denoting by $\langle,\rangle_{j}$ the metric induced by $f_{j}$, we obtain

$$
\begin{aligned}
\left\langle\bar{\omega}_{i}^{t}\left(v_{i}\right), X\right\rangle_{j} & =\left\langle v_{i}, \omega_{i}\left(D_{j} X\right)-\Omega_{i j} \Omega_{j j}^{-1} \omega_{j}\left(D_{j} X\right)\right\rangle \\
& =\left\langle D_{j} \omega_{i}^{t}\left(v_{i}\right)-D_{j} \omega_{j}^{t}\left(\Omega_{j j}^{-1}\right)^{t} \Omega_{i j}^{t}\left(v_{i}\right), X\right\rangle \\
& =\left\langle\omega_{i}^{t}\left(v_{i}\right)-\omega_{j}^{t}\left(\Omega_{j j}^{-1}\right)^{t} \Omega_{i j}^{t}\left(v_{i}\right), D_{j}^{-1} X\right\rangle_{j} .
\end{aligned}
$$


Since $D_{j}^{-1}$ is symmetric with respect to $\langle,\rangle_{j}$, it follows that $D_{j} \bar{\omega}_{i}^{t}=\omega_{i}^{t}-\omega_{j}^{t}\left(\Omega_{j j}^{-1}\right)^{t} \Omega_{i j}^{t}$. Using (21) we get

$$
\begin{aligned}
D_{j}\left(\nabla_{X} \bar{\omega}_{i}^{t}\right)\left(v_{i}\right)= & D_{j} \nabla_{X}^{j} \bar{\omega}_{i}^{t}\left(v_{i}\right)-D_{j} \bar{\omega}_{i}^{t}\left(\nabla_{X}^{V_{i}} v_{i}\right) \\
= & \nabla_{X} \omega_{i}^{t}\left(v_{i}\right)-\nabla_{X} \omega_{j}^{t}\left(\Omega_{j j}^{-1}\right)^{t} \Omega_{i j}^{t}\left(v_{i}\right)-\Phi_{j}(X) \Omega_{j j}^{-1} \omega_{j} \omega_{i}^{t}\left(v_{i}\right) \\
& +\Phi_{j}(X) \Omega_{j j}^{-1} \omega_{j} \omega_{j}^{t}\left(\Omega_{j j}^{-1}\right)^{t} \Omega_{i j}^{t}\left(v_{i}\right)+\omega_{j}^{t}\left(\Omega_{j j}^{-1}\right)^{t} \Phi_{j}(X)^{t} \omega_{i}^{t}\left(v_{i}\right) \\
& -\omega_{j}^{t}\left(\Omega_{j j}^{-1}\right)^{t} \Phi_{j}(X)^{t} \omega_{j}^{t}\left(\Omega_{j j}^{-1}\right)^{t} \Omega_{i j}^{t}\left(v_{i}\right)+\omega_{j}^{t}\left(\Omega_{j j}^{-1}\right)^{t} \Omega_{i j}^{t}\left(\nabla_{X}^{V_{i}} v_{i}\right)-\omega_{i}^{t}\left(\nabla_{X}^{V_{i}} v_{i}\right) .
\end{aligned}
$$

Now,

$$
\begin{aligned}
-\nabla_{X}^{j} \omega_{j}^{t}\left(\Omega_{j j}^{-1}\right)^{t} \Omega_{i j}^{t}\left(v_{i}\right) & +\omega_{j}^{t}\left(\Omega_{j j}^{-1}\right)^{t} \Omega_{i j}^{t}\left(\nabla_{X}^{V_{i}} v_{i}\right)=-\left(\nabla_{X} \omega_{j}^{t}\right)\left(\Omega_{j j}^{-1}\right)^{t} \Omega_{i j}^{t}\left(v_{i}\right) \\
& +\omega_{j}^{t}\left(\Omega_{j j}^{-1}\right)^{t} \Phi_{j}(X)^{t} \omega_{j}^{t}\left(\Omega_{j j}^{-1}\right)^{t} \Omega_{i j}^{t}\left(v_{i}\right)-\omega_{j}^{t}\left(\Omega_{j j}^{-1}\right)^{t} \Phi_{j}(X)^{t} \omega_{i}^{t}\left(v_{i}\right) .
\end{aligned}
$$

Therefore

$$
D_{j}\left(\nabla_{X} \bar{\omega}_{i}^{t}\right)=\nabla_{X} \omega_{i}^{t}-\left(\nabla_{X} \omega_{j}^{t}\right)\left(\Omega_{j j}^{-1}\right)^{t} \Omega_{i j}^{t}-\Phi_{j}(X) \Omega_{j j}^{-1} \omega_{j} \omega_{i}^{t}+\Phi_{j}(X) \Omega_{j j}^{-1} \omega_{j} \omega_{j}^{t}\left(\Omega_{j j}^{-1}\right)^{t} \Omega_{i j}^{t} .
$$

On the other hand, from (19) we have

$$
D_{j} A^{j}(X) \bar{\beta}_{i}=A(X) \beta_{i}-A(X) \beta_{j}\left(\Omega_{j j}^{-1}\right)^{t} \Omega_{i j}^{t}+\Phi_{j}(X) \Omega_{j j}^{-1} \beta_{j}^{t} \beta_{i}-\Phi_{j}(X) \Omega_{j j}^{-1} \beta_{j}^{t} \beta_{j}\left(\Omega_{j j}^{-1}\right)^{t} \Omega_{i j}^{t} .
$$

Also,

$$
\begin{aligned}
D_{j}(X) \bar{\varphi}_{i}^{t} & =\left(X-\Phi_{j}(X) \Omega_{j j}^{-1} \varphi_{j}\right)\left(\varphi_{i}^{t}-\varphi_{j}^{t}\left(\Omega_{j j}^{-1}\right)^{t} \Omega_{i j}^{t}\right) \\
& =X \varphi_{i}^{t}-X \varphi_{j}^{t}\left(\Omega_{j j}^{-1}\right)^{t} \Omega_{i j}{ }^{t}-\Phi_{j}(X) \Omega_{j j}^{-1} \varphi_{j} \varphi_{i}^{t}+\Phi_{j}(X) \Omega_{j j}^{-1} \varphi_{j} \varphi_{j}^{t}\left(\Omega_{j j}^{-1}\right)^{t} \Omega_{i j}^{t} .
\end{aligned}
$$

Thus

$$
\begin{aligned}
D_{j} \bar{\Phi}_{i}(X)= & \Phi_{i}(X)-\Phi_{j}(X)\left(\Omega_{j j}^{-1}\right)^{t} \Omega_{i j}^{t}-\Phi_{j}(X) \Omega_{j j}^{-1} \omega_{j} \omega_{i}^{t}+\Phi_{j}(X) \Omega_{j j}^{-1} \omega_{j} \omega_{j}^{t}\left(\Omega_{j j}^{-1}\right)^{t} \Omega_{i j}^{t} \\
& -\Phi_{j}(X) \Omega_{j j}^{-1} \beta_{j}^{t} \beta_{i}+\Phi_{j}(X) \Omega_{j j}^{-1} \beta_{j}^{t} \beta_{j}\left(\Omega_{j j}^{-1}\right)^{t} \Omega_{i j}^{t}-\tilde{c} \Phi_{j}(X) \Omega_{j j}^{-1} \varphi_{j} \varphi_{i}^{t} \\
& +\tilde{c} \Phi_{j}(X) \Omega_{j j}^{-1} \varphi_{j} \varphi_{j}^{t}\left(\Omega_{j j}^{-1}\right)^{t} \Omega_{i j}^{t} .
\end{aligned}
$$

Using that $\mathcal{G}_{j}^{t} \mathcal{G}_{j}=\omega_{j} \omega_{j}^{t}+\beta_{j}^{t} \beta_{j}+\tilde{c} \varphi_{j} \varphi_{j}^{t}$ and $\mathcal{G}_{j}^{t} \mathcal{G}_{i}=\omega_{j} \omega_{i}^{t}+\beta_{j}^{t} \beta_{i}+\tilde{c} \varphi_{j} \varphi_{i}^{t}$ we obtain

$$
\begin{aligned}
\left(\Omega_{j j}^{-1}\right)^{t} \Omega_{i j}^{t}+\Omega_{j j}^{-1} \omega_{j} \omega_{i}^{t} & -\Omega_{j j}^{-1} \omega_{j} \omega_{j}^{t}\left(\Omega_{j j}^{-1}\right)^{t} \Omega_{i j}^{t}+\Omega_{j j}^{-1} \beta_{j}^{t} \beta_{i}-\Omega_{j j}^{-1} \beta_{j}^{t} \beta_{j}\left(\Omega_{j j}^{-1}\right)^{t} \Omega_{i j}^{t} \\
& +\tilde{c} \Omega_{j j}^{-1} \varphi_{j} \varphi_{i}^{t}-\tilde{c} \Omega_{j j}^{-1} \varphi_{j} \varphi_{j}^{t}\left(\Omega_{j j}^{-1}\right)^{t} \Omega_{i j}^{t} \\
& =\left(\Omega_{j j}^{-1}\right)^{t} \Omega_{i j}^{t}+\Omega_{j j}^{-1} \mathcal{G}_{j}^{t} \mathcal{G}_{i}-\Omega_{j j}^{-1} \mathcal{G}_{j}^{t} \mathcal{G}_{j}\left(\Omega_{j j}^{-1}\right)^{t} \Omega_{i j}^{t} \\
& =\left(\Omega_{j j}^{-1}\right)^{t} \Omega_{i j}^{t}+\Omega_{j j}^{-1} \Omega_{i j}^{t}+\Omega_{j j}^{-1} \Omega_{j i}-\left(\Omega_{j j}^{-1}\right)^{t} \Omega_{i j}^{t}-\Omega_{j j}^{-1} \Omega_{i j}^{t} \\
& =\Omega_{j j}^{-1} \Omega_{j i} .
\end{aligned}
$$

Substituting this into (55) yields (53). 
Theorem 31. Let $f: M^{n}(c) \rightarrow \mathbb{Q}^{n+p}(\tilde{c}),(c, \tilde{c}) \in D(c, \tilde{c})$, be an isometric immersion and let $\mathcal{R}_{\varphi, \beta, \Omega, L}(f): \tilde{M}^{n} \rightarrow \mathbb{Q}^{n+p}(\tilde{c})$ be an L-transform of $f$. Assume that $L$ decomposes as $L=L_{1} \oplus L_{2}$ with respect to an orthogonal decomposition $V=V_{1} \oplus V_{2}$. If $\left(\varphi_{j}, \beta_{j}, \Omega_{i j}\right)$ and $\left(\bar{\varphi}_{i}, \bar{\beta}_{i}, \bar{\Omega}_{i i}\right)$ are given as in (22) and (23), respectively, then $\left(\varphi_{j}, \beta_{j}, \Omega_{j j}, L_{j}\right)$ defines an $L_{j}$-transform of $f$ for $1 \leq j \leq 2,\left(\bar{\varphi}_{i}, \bar{\beta}_{i}, \bar{\Omega}_{i i}, L_{i}\right)$ an $L_{i}$-transform of $f_{j}$ for $1 \leq i \neq j \leq 2$, and

$$
\mathcal{R}_{\varphi, \beta, \Omega, L}(f)=\mathcal{R}_{\bar{\varphi}_{i}, \bar{\beta}_{i}, \bar{\Omega}_{i i}, L_{i}}\left(\mathcal{R}_{\varphi_{j}, \beta_{j}, \Omega_{j j}, L_{j}}(f)\right) .
$$

The same conclusion holds if $(c, \tilde{c}) \notin D(c, \tilde{c})$ as long as $\Omega_{j j}$ is invertible for $1 \leq j \leq 2$.

Proof: Since $\mathcal{R}_{\varphi, \beta, \Omega, L}(f)$ is an $L$-transform of $f$, equations (311) and (32) hold. By the assumption that $L=L_{1} \oplus L_{2}$, these equations are equivalent to

$$
\Theta_{j}(X):=\Phi_{j}(X) L_{j}+A(X) \beta_{j}+(c-\tilde{c}) X \varphi_{j}^{t}=0
$$

and

$$
\Omega_{i j} L_{j}+L_{i}^{t} \Omega_{j i}^{t}-\beta_{i}^{t} \beta_{j}+(c-\tilde{c}) \varphi_{i} \varphi_{j}^{t}=0,
$$

$1 \leq i, j \leq 2$. Therefore, in order to prove that $\left(\varphi_{j}, \beta_{j}, \Omega_{j j}, L_{j}\right)$ defines an $L_{j}$-transform of $f$ for $1 \leq j \leq 2$ it remains to show that $\Omega_{j j}$ is invertible for $1 \leq j \leq 2$. Assume otherwise, say, that $\operatorname{ker} \Omega_{11} \neq\{0\}$. Let $\alpha_{1}, \ldots, \alpha_{p}$ (resp., $\gamma_{1}, \ldots, \gamma_{q}$ ) be the real (resp., complex) eigenvalues of $L$, and let $a_{i 1}, a_{i 2}, \ldots, a_{i n_{i}}$ (resp., $w_{j 1}, w_{j 2}, \ldots, w_{j m_{j}}$ ) be the generalized eigenvectors of $L$ associated to $\alpha_{i}, 1 \leq i \leq p$ (resp., $\left.\gamma_{j}, 1 \leq j \leq q\right)$. Since $(c, \tilde{c}) \in D(c, \tilde{c})$, by Proposition 21 either there exists $1 \leq i \leq p$ such that $a_{i 1} \in \operatorname{ker} \Omega_{11} \subset S_{1}$, where $S_{1}$ is defined as in (40) for $\left(\psi_{1}=\left.\varphi^{t}\right|_{V_{1}}, \nu_{1}=\left.\omega^{t}\right|_{V_{1}}, \beta_{1}=\left.\beta\right|_{V_{1}}\right)$, or there exists $1 \leq j \leq q$ such that $a_{j 1} \in\left(\operatorname{ker} \Omega_{11}\right)^{c} \subset S_{1}^{c}$. We argue for the first possibility, the argument for the second one being similar.

Thus we have $L a_{i 1}=\alpha_{i} a_{i 1}$, with $a_{i 1} \in S \cap V_{1}$. Since $\operatorname{span}\left\{a_{i 1}, \ldots, a_{i n_{i}}\right\} \subset V_{1}$, we have

$$
0=\left\langle\Omega_{11} a_{i 1}, a_{i \ell}\right\rangle=\left\langle\Omega a_{i 1}, a_{i \ell}\right\rangle, \quad 1 \leq \ell \leq n_{i} .
$$

On the other hand, from $\Omega+\Omega^{t}=\mathcal{G}^{t} \mathcal{G}$ and (32) that $\Omega_{s} a_{i 1}=0$ and $L^{t} \Omega_{a} a_{i 1}=\alpha_{i} \Omega_{a} a_{i 1}$. Thus, for all real eigenvalues $\alpha_{j}, 1 \leq j \leq p$, with $\alpha_{j} \neq \alpha_{i}$, and all complex eigenvalues $\gamma_{j}$, $1 \leq j \leq q$, we have

$$
\begin{aligned}
\alpha_{j}\left\langle\Omega_{a} a_{i 1}, a_{j t}\right\rangle & =\left\langle\Omega_{a} a_{i 1}, L a_{j t}-a_{j, t-1}\right\rangle \\
& =\left\langle L^{t} \Omega_{a} a_{i 1}, a_{j t}\right\rangle-\left\langle\Omega_{a} a_{i 1}, a_{j, t-1}\right\rangle \\
& =\alpha_{i}\left\langle\Omega_{a} a_{i 1}, a_{j t}\right\rangle-\left\langle\Omega_{a} a_{i 1}, a_{j, t-1}\right\rangle, a_{j 0}=0, \\
\bar{\gamma}_{j}\left\langle\Omega_{a} a_{i 1}, w_{j k}\right\rangle & =\left\langle\Omega_{a} a_{i 1}, L w_{j k}-w_{j, k-1}\right\rangle \\
& =\left\langle L^{t} \Omega_{a} a_{i 1}, w_{j k}\right\rangle-\left\langle\Omega_{a} a_{i 1}, w_{j, k-1}\right\rangle \\
& =\alpha_{i}\left\langle\Omega_{a} a_{i 1}, w_{j k}\right\rangle-\left\langle\Omega_{a} a_{i 1}, w_{j, k-1}\right\rangle, w_{j 0}=0 .
\end{aligned}
$$


It follows from (58), (59), (60) and $\Omega_{s} a_{i 1}=0$ that $\Omega a_{i 1}=0$, a contradiction.

We now show that $\left(\bar{\varphi}_{i}, \bar{\beta}_{i}, \bar{\Omega}_{i i}, L_{i}\right)$ defines an $L_{i}$-transform of $f_{j}$ for $1 \leq i \neq j \leq 2$. We must prove that

$$
\bar{\Theta}_{i}(X):=\bar{\Phi}_{i}(X) L_{i}+A^{j}(X) \bar{\beta}_{i}+(c-\tilde{c}) X \bar{\varphi}_{i}^{t}=0=\bar{\Omega}_{i i} L_{i}+L_{i}^{t} \bar{\Omega}_{i i}^{t}-\left(\bar{\beta}_{i}^{t} \bar{\beta}_{i}-(c-\tilde{c}) \bar{\varphi}_{i} \bar{\varphi}_{i}^{t}\right) .
$$

Using (19), (153), (56) and (57) we obtain

$$
\begin{aligned}
D_{j} \bar{\Theta}_{i}(X)= & \Phi_{i}(X) L_{i}-\Phi_{j}(X) \Omega_{j j}^{-1} \Omega_{j i} L_{i}+D_{j} A^{j}(X) \mathcal{P}_{j}\left(\beta_{i}-\beta_{j}\left(\Omega_{j j}^{-1}\right)^{t} \Omega_{i j}^{t}\right) \\
& +(c-\tilde{c}) D_{j}(X)\left(\varphi_{i}-\Omega_{i j} \Omega_{j j}^{-1} \varphi_{j}\right)^{t} \\
= & \Phi_{i}(X) L_{i}-\Phi_{j}(X) \Omega_{j j}^{-1} \Omega_{j i} L_{i}+\left(A(X)+\Phi_{j}(X) \Omega_{j j}^{-1} \beta_{j}^{t}\right)\left(\beta_{i}-\beta_{j}\left(\Omega_{j j}^{-1}\right)^{t} \Omega_{i j}^{t}\right) \\
& +(c-\tilde{c})\left(X-\Phi_{j}(X) \Omega_{j j}^{-1} \varphi_{j}\right)\left(\varphi_{i}^{t}-\varphi_{j}^{t}\left(\Omega_{j j}^{-1}\right)^{t} \Omega_{i j}^{t}\right) \\
= & \Phi_{i}(X) L_{i}+A(X) \beta_{i}+(c-\tilde{c}) X \varphi_{i}^{t}-\Phi_{j}(X) \Omega_{j j}^{-1}\left(\Omega_{j i} L_{i}-\beta_{j}^{t} \beta_{i}+(c-\tilde{c}) \varphi_{j} \varphi_{i}^{t}\right) \\
& +\left(\Phi_{j}(X) \Omega_{j j}^{-1}\left(-\beta_{j}^{t} \beta_{j}+(c-\tilde{c}) \varphi_{j} \varphi_{j}^{t}\right)-A(X) \beta_{j}-(c-\tilde{c}) X \varphi_{j}^{t}\right)\left(\Omega_{j j}^{-1}\right)^{t} \Omega_{i j}^{t}=0 .
\end{aligned}
$$

On the other hand,

$$
\begin{aligned}
\bar{\Omega}_{i i} L_{i}+ & L_{i}^{t} \bar{\Omega}_{i i}^{t}-\left(\bar{\beta}_{i}^{t} \bar{\beta}_{i}-(c-\tilde{c}) \bar{\varphi}_{i} \bar{\varphi}_{i}^{t}\right) \\
& =\left(\Omega_{i i}-\Omega_{i j} \Omega_{j j}^{-1} \Omega_{j i}\right) L_{i}+L_{i}^{t}\left(\Omega_{i i}^{t}-\Omega_{j i}^{t}\left(\Omega_{j j}^{-1}\right)^{t} \Omega_{i j}^{t}\right) \\
& -\left(\beta_{i}^{t}-\Omega_{i j} \Omega_{j j}^{-1} \beta_{j}^{t}\right)\left(\beta_{i}-\beta_{j}\left(\Omega_{j j}^{-1}\right)^{t} \Omega_{i j}^{t}\right)+(c-\tilde{c})\left(\varphi_{i}-\Omega_{i j} \Omega_{j j}^{-1} \varphi_{j}\right)\left(\varphi_{i}^{t}-\varphi_{j}^{t}\left(\Omega_{j j}^{-1}\right)^{t} \Omega_{i j}^{t}\right) \\
& =\Omega_{i i} L_{i}+L_{i}^{t} \Omega_{i i}^{t}-\beta_{i}^{t} \beta_{i}+(c-\tilde{c}) \varphi_{i} \varphi_{i}^{t}-\Omega_{i j} \Omega_{j j}^{-1}\left(\Omega_{j i} L_{i}-\beta_{j}^{t} \beta_{i}+(c-\tilde{c}) \varphi_{j} \varphi_{i}^{t}\right) \\
& -\left(L_{i}^{t} \Omega_{j i}^{t}-\beta_{i}^{t} \beta_{j}+(c-\tilde{c}) \varphi_{i} \varphi_{j}^{t}\right)\left(\Omega_{j j}^{-1}\right)^{t} \Omega_{i j}^{t}-\Omega_{i j} \Omega_{j j}^{-1}\left(\beta_{j}^{t} \beta_{j}-(c-\tilde{c}) \varphi_{j} \varphi_{j}^{t}\right)\left(\Omega_{j j}^{-1}\right)^{t} \Omega_{i j}^{t} \\
& =\Omega_{i j} \Omega_{j j}^{-1} L_{j}^{t} \Omega_{i j}^{t}+\Omega_{i j} L_{j}\left(\Omega_{j j}^{-1}\right)^{t} \Omega_{i j}^{t}-\Omega_{i j} \Omega_{j j}^{-1}\left(\Omega_{j j} L_{j}+L_{j}^{t} \Omega_{j j}^{t}\right)\left(\Omega_{j j}^{-1}\right)^{t} \Omega_{i j}^{t}=0 . \mathbf{\square}
\end{aligned}
$$

Remarks 32. (i) In Theorem 31, if $L$ decomposes as $L=L_{1} \oplus L_{2}$ with respect to any direct sum decomposition $V=V_{1} \oplus V_{2}$, then one can always assume the decomposition to be orthogonal after a suitable change of the inner product on $V$. Namely, define $W_{i}=V_{j}^{\perp}$, $1 \leq i \neq j \leq 2$, and let $B \in V^{*} \otimes V$ be an invertible endomorphism such that $B W_{i}=V_{i}$, $1 \leq i \leq 2$. Let $\langle\cdot, \cdot\rangle^{\sim}$ be the inner product on $V$ given by $\langle\cdot, \cdot\rangle^{\sim}=\langle\cdot, B \cdot\rangle$. Then $W_{1}$ and $W_{2}$ are orthogonal with respect to $\langle\cdot, \cdot\rangle^{\sim}$, because $W_{1}$ and $B W_{2}=V_{2}$ are orthogonal with respect to $\langle\cdot, \cdot\rangle$. Moreover, $\hat{L}=B^{-1} L B$ decomposes as $\hat{L}=\hat{L}_{1} \oplus \hat{L}_{2}$ with respect to the decomposition $V=W_{1} \oplus W_{2}$, for

$$
\hat{L} W_{i}=B^{-1} L B W_{i}=B^{-1} L V_{i} \subset B^{-1} V_{i}=W_{i}, \quad 1 \leq i \leq 2 .
$$

(ii) In particular, let $\tilde{f}=\mathcal{R}_{\varphi, \beta, \Omega, L}(f)$ be an $L$-transform of $f$ such that $L$ is diagonalizable, with $v_{1}, \ldots, v_{k}$ as a basis of eigenvectors. If $e_{1}, \ldots, e_{k} \in V$ are such that $\left\langle e_{i}, v_{j}\right\rangle=\delta_{i}^{j}$, 
$1 \leq i, j \leq k$, and we define $B \in V^{*} \otimes V$ by $B e_{i}=v_{i}, 1 \leq i \leq k$, then $e_{1}, \ldots, e_{k}$ is a basis of eigenvectors of $\hat{L}=B^{-1} L B$ that is orthonormal with respect to $\langle\cdot, \cdot\rangle^{\sim}=\langle\cdot, B \cdot\rangle$. Thus, if $\mathcal{R}_{\varphi, \beta, \Omega, L}(f)$ is an $L$-transform of $f$ such that $L$ is diagonalizable, we may always assume that $L$ is symmetric after an appropriate change of the inner product on $V$. It follows from Theorem 31 that an $L$-transformation can be obtained by an iteration of scalar $L_{i}$-transformations, $1 \leq i \leq k=\operatorname{dim} V$, if and only if $L$ is diagonalizable.

\subsection{The Bianchi $L$-cube}

Given $L_{1} \neq L_{2} \in \mathbb{R}$, we say that a Bianchi quadrilateral $\left\{f, f_{1}, f_{2}, f_{12}\right\}$ is a Bianchi $\left(L_{1}, L_{2}\right)$-quadrilateral if the metric induced by $f$ has constant curvature $c, f_{i}$ is an $L_{i^{-}}$ transform of $f, 1 \leq i \leq 2$, and $f_{12}$ is an $L_{2}$-transform of $f_{1}$ and an $L_{1}$-transform of $f_{2}$.

We will need the following fact proved in [6], which can also be derived from Theorem 31 ,

Proposition 33. Let $f: M^{n}(c) \rightarrow \mathbb{Q}^{n+p}(\tilde{c}),(c, \tilde{c}) \in D(c, \tilde{c})$, be an isometric immersion. If $f_{i}=\mathcal{R}_{\varphi_{i}, \beta_{i}}(f): M_{i}^{n}(c) \rightarrow \mathbb{Q}^{n+p}(\tilde{c})$ is an $L_{i}$-transform of $f, 1 \leq i \leq 2$, with $L_{1} \neq L_{2}$ and $\left[A_{\beta_{1}}, A_{\beta_{2}}\right]=0$, then there exists exactly one isometric immersion $\tilde{f}: \tilde{M}^{n}(c) \rightarrow \mathbb{Q}^{n+p}(\tilde{c})$ such that $\left\{f, f_{1}, f_{2}, \tilde{f}\right\}$ is a Bianchi $\left(L_{1}, L_{2}\right)$-quadrilateral.

Given $L_{1}, \ldots, L_{k} \in \mathbb{R}$, with $L_{i} \neq L_{j}$ for all $1 \leq i \neq j \leq k$, we say that a Bianchi cube $\left(\mathcal{C}_{0}, \ldots, \mathcal{C}_{k}\right)$ is a Bianchi $\left(L_{1}, \ldots, L_{k}\right)$-cube if, for all $1 \leq s \leq k-1$,

(i) Each $f_{\alpha_{s+1}} \in \mathcal{C}_{s+1}$ with $\alpha_{s+1}=\alpha_{s} \cup\left\{i_{j}\right\}$ is an $L_{i_{j}}$-transform of $f_{\alpha_{s}} \in \mathcal{C}_{s}$.

(ii) $\left\{f_{\alpha_{s-1}}, f_{\alpha_{s-1} \cup\left\{i_{l}\right\}}, f_{\alpha_{s-1} \cup\left\{i_{j}\right\}}, f_{\alpha_{s+1}}\right\}$ is a Bianchi $\left(L_{i_{l}}, L_{i_{j}}\right)$-quadrilateral when $\alpha_{s+1}=$ $\alpha_{s-1} \cup\left\{i_{l}, i_{j}\right\}$.

Theorem 34. Let $f: M^{n}(c) \rightarrow \mathbb{Q}^{n+p}(\tilde{c}),(c, \tilde{c}) \in D(c, \tilde{c})$, be an isometric immersion. If $f_{i}=\mathcal{R}_{\varphi_{i}, \beta_{i}}(f): M_{i}^{n}(c) \rightarrow \mathbb{Q}^{n+p}(\tilde{c}), 1 \leq i \leq k$, are $L_{i}$-transforms of $f$ such that $L_{i} \neq L_{j}$ and $\left[A_{\beta_{i}}, A_{\beta_{j}}\right]=0$ for all $1 \leq i \neq j \leq k$, then there exists a Bianchi $\left(L_{1}, \ldots, L_{k}\right)$-cube $\left(\mathcal{C}_{0}, \ldots, \mathcal{C}_{k}\right)$ such that $\mathcal{C}_{0}=\{f\}$ and $\mathcal{C}_{1}=\left\{f_{1}, \ldots, f_{k}\right\}$, which is unique if no $f_{i}$ belongs to the associated family determined by $\left\{f_{j}, f_{l}\right\}$ for all $1 \leq i \neq j \neq l \neq i \leq k$.

Proof: We first prove existence. Set $F=i \circ f$ and $\mathcal{G}_{i}=F_{*} \nabla \varphi_{i}+i_{*} \beta_{i}+\tilde{c} \varphi_{i} F$. Since $f_{i}$ is an $L_{i}$-transform of $f$, for each $1 \leq i \leq k$ the pair $\left(\varphi_{i}, \beta_{i}\right)$ satisfies

$$
\alpha\left(X, \nabla \varphi_{i}\right)+\nabla_{X}^{\perp} \beta_{i}=0
$$

for all $X \in \mathfrak{X}(M)$ and the tensor $\Phi_{i}=\operatorname{Hess} \varphi_{i}-A_{\beta_{i}}-\tilde{c} \varphi_{i} I$ satisfies

$$
L_{i} \Phi_{i}+A_{\beta_{i}}+(c-\tilde{c}) \varphi_{i} I=0 .
$$


Moreover, $L_{i}\left|\mathcal{G}_{i}\right|^{2}=\left|\beta_{i}\right|^{2}-(c-\tilde{c}) \varphi_{i}^{2}$. Define $\varphi: M^{n}(c) \rightarrow \mathbb{R}^{k}, \beta \in \Gamma\left(\left(\mathbb{R}^{k}\right)^{*} \otimes N_{f} M\right)$ and $\Omega \in \Gamma\left(\left(\mathbb{R}^{k}\right)^{*} \otimes \mathbb{R}^{k}\right)$ by

$$
\varphi=\left(\varphi_{1}, \ldots, \varphi_{k}\right), \quad \beta=\sum_{i=1}^{k} e^{i} \otimes \beta_{i} \text { and } \Omega=\sum \Omega_{i j} e^{j} \otimes e_{i}
$$

where $e_{1}, \ldots, e_{k}$ is the canonical basis of $\mathbb{R}^{k}, e^{1}, \ldots, e^{k}$ is its dual basis,

$$
\Omega_{i j}=\frac{1}{L_{j}-L_{i}}\left(\rho_{i j}-L_{i}\left\langle\mathcal{G}_{i}, \mathcal{G}_{j}\right\rangle\right), \quad i \neq j, \text { and } \Omega_{i i}=\frac{1}{2}\left|\mathcal{G}_{i}\right|^{2}, \quad 1 \leq i \leq k,
$$

where $\rho_{i j}=\left\langle\beta_{i}, \beta_{j}\right\rangle-(c-\tilde{c}) \varphi_{i} \varphi_{j}$.

We claim that $(\varphi, \beta, \Omega)$ defines an $L$-transformation of $f$, where $L \in\left(\mathbb{R}^{k}\right)^{*} \otimes \mathbb{R}^{k}$ is given by $L=\sum_{i=1}^{k} L_{i} e^{i} \otimes e_{i}$. First, that $(\varphi, \beta)$ satisfies (5) follows from (61). The comutativity relations (6) follow from the assumption that $\left[A_{\beta_{i}}, A_{\beta_{j}}\right]=0, i \neq j$, because $\Phi=\Phi(\varphi, \beta)$ satisfies (31) in view of (62).

Equation (15) is equivalent to

$$
X\left(\Omega_{i j}\right)=\left\langle\nabla \varphi_{i}, \Phi_{j} X\right\rangle
$$

for all $X \in \mathfrak{X}(M)$ and $1 \leq i, j \leq k$. Now,

$$
\begin{aligned}
X\left(\rho_{i j}\right) & =\left\langle\nabla_{X}^{\perp} \beta_{i}, \beta_{j}\right\rangle+\left\langle\beta_{i}, \nabla_{X}^{\perp} \beta_{j}\right\rangle-(c-\tilde{c})\left\langle\nabla \varphi_{i}, X\right\rangle \varphi_{j}-(c-\tilde{c})\left\langle\nabla \varphi_{j}, X\right\rangle \varphi_{i} \\
& =-\left\langle\left(A_{\beta_{j}}^{f}+(c-\tilde{c}) \varphi_{j} I\right) X, \nabla \varphi_{i}\right\rangle-\left\langle\left(A_{\beta_{i}}^{f}+(c-\tilde{c}) \varphi_{i} I\right) X, \nabla \varphi_{j}\right\rangle
\end{aligned}
$$

and

$$
\begin{aligned}
X\left(\left\langle\mathcal{G}_{i}, \mathcal{G}_{j}\right\rangle\right) & =\left\langle\mathcal{G}_{i *} X, \mathcal{G}_{j}\right\rangle+\left\langle\mathcal{G}_{i}, \mathcal{G}_{j_{*}} X\right\rangle \\
& =\left\langle\Phi_{i} X, \nabla \varphi_{j}\right\rangle+\left\langle\nabla \varphi_{i}, \Phi_{j} X\right\rangle .
\end{aligned}
$$

Since

$$
X\left(\Omega_{i j}\right)=\frac{1}{\left(L_{j}-L_{i}\right)}\left(X\left(\rho_{i j}\right)-L_{i} X\left(\left\langle\mathcal{G}_{i}, \mathcal{G}_{j}\right\rangle\right)\right),
$$

(64) follows from (62), (65) and (66). Finally, equation (63) implies (16) and (32).

Since $\Omega_{i i}$ is nowhere vanishing for $1 \leq i \leq k$, if $c \neq \tilde{c}$ (respectively, $c=\tilde{c}$ ) then $\varphi_{i}$ and $\beta_{i}$ (resp., $\beta_{i}$ ) do not vanish simultaneously (resp., does not vanish) at any point. In particular, no $e_{i}, 1 \leq i \leq k$, belongs to the subspace $S$ defined by (40). It follows from Proposition 21 that $\left(\varphi^{t}, \omega^{t}, \beta\right)$ is $L$-admissible, that is, $\Omega$ is invertible. Thus $\tilde{f}=\mathcal{R}_{\varphi, \beta, \Omega}(f)$ is an $L$-transform of $f$. 
For any multi-index $\alpha_{r}=\left\{i_{1}, \ldots, i_{r}\right\} \in \Lambda_{r}$, define

$$
\varphi^{\alpha_{r}}=\sum_{j=1}^{r} \varphi_{i_{j}} e_{i_{j}}, \beta^{\alpha_{r}}=\sum_{j=1}^{r} e^{i_{j}} \otimes \beta_{i_{j}}, \Omega^{\alpha_{r}}=\sum_{\ell, j=1}^{r} \Omega_{i_{\ell} i_{j}} e^{i_{j}} \otimes e_{i_{\ell}}, L^{\alpha_{r}}=\sum_{\ell, j=1}^{r} L_{i_{\ell} i_{j}} e^{i_{\ell}} \otimes e_{i_{j}} .
$$

By Theorem 31, for each $\alpha_{r} \in \Lambda_{r}$ the triple $\left(\varphi^{\alpha_{r}}, \beta^{\alpha_{r}}, \Omega^{\alpha_{r}}\right)$ defines an $L^{\alpha_{r}}$-transform of $f$. Let $\mathcal{C}_{r}$ be the family of $\left(\begin{array}{l}k \\ r\end{array}\right)$ elements formed by the $L^{\alpha_{r}}$-vectorial Ribaucour transforms

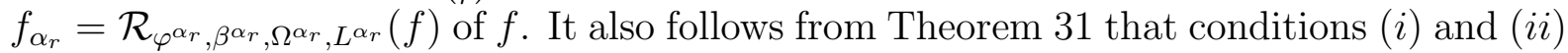
in the definition of a Bianchi $\left(L_{1}, \ldots, L_{k}\right)$-cube are satisfied by the $(k+1)$-tuple $\left(\mathcal{C}_{0}, \ldots, \mathcal{C}_{k}\right)$.

For the uniqueness, first notice that, by Proposition 33, for each pair $\{i, j\} \in\{1, \ldots, k\}$ with $i \neq j$ there is a unique $f_{i j}$ such that $\left\{f, f_{i}, f_{j}, f_{i j}\right\}$ is a Bianchi $\left(L_{i}, L_{j}\right)$-quadrilateral. By Theorem 8 , there exists a unique Bianchi $k$-cube $\left(\mathcal{C}_{0}, \ldots, \mathcal{C}_{k}\right)$ such that $\mathcal{C}_{0}=\{f\}, \mathcal{C}_{1}=$ $\left\{f_{1}, \ldots, f_{k}\right\}$ and $\mathcal{C}_{2}=\left\{f_{i j}\right\}_{1 \leq i \neq j \leq k}$.

Remark 35. ( $i$ ) It is worth pointing out that, once the $L_{i}$-transforms $f_{i}=\mathcal{R}_{\varphi_{i}, \beta_{i}}(f)$, $1 \leq i \leq k$, of $f: M^{n}(c) \rightarrow \mathbb{Q}^{n+p}(\tilde{c})$ are known (meaning that all the pairs $\left(\varphi_{i}, \beta_{i}\right), 1 \leq$ $i \leq k$, are known), and hence the first two families $\mathcal{C}_{0}=\{f\}$ and $\mathcal{C}_{1}=\left\{f_{1}, \ldots, f_{k}\right\}$ in the Bianchi $\left(L_{1}, \ldots, L_{k}\right)$-cube $\left(\mathcal{C}_{0}, \ldots, \mathcal{C}_{k}\right)$ are given, then all $2^{k}-(k+1)$ elements in the remaining families $\mathcal{C}_{2}, \ldots, \mathcal{C}_{k}$ are determined by explicit algebraic formulae, with no integrations required. Namely, if $\Omega \in \Gamma\left(\left(\mathbb{R}^{k}\right)^{*} \otimes \mathbb{R}^{k}\right)$ is defined by $\Omega=\sum \Omega_{i j} e^{j} \otimes e_{i}$, where the $\Omega_{i j}, 1 \leq i, j \leq k$, are given by the algebraic expressions (63), which only depend on the $\left(\varphi_{i}, \beta_{i}\right), 1 \leq i \leq k$, then all elements of $\mathcal{C}_{r}, 2 \leq r \leq k$, are given explicitly by

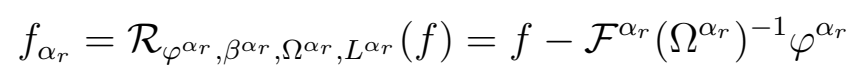

where $\alpha_{r} \in\left\{i_{1}, \ldots, i_{r}\right\} \in \Lambda_{r},\left(\varphi^{\alpha_{r}}, \beta^{\alpha_{r}}, \Omega^{\alpha_{r}}\right)$ is given by (67) and $\mathcal{F}^{\alpha_{r}}=f_{*}\left(d \varphi^{\alpha_{r}}\right)^{t}+\beta^{\alpha_{r}}$.

(ii) In Theorem [34, if $f_{i}$ belongs to the associated family determined by $\left\{f_{j}, f_{l}\right\}, 1 \leq i \neq$ $j \neq l \neq i \leq k$, that is, there exist $a_{j}, a_{l} \in \mathbb{R}$ such that $\varphi_{i}=a_{j} \varphi_{j}+a_{l} \varphi_{l}$ and $\beta_{i}=a_{j} \beta_{j}+a_{l} \beta_{l}$, then from $\Phi_{i}=a_{j} \Phi_{j}+a_{l} \Phi_{l}$ and (62) one obtains that $A_{\xi}=\lambda I$ for

$$
\xi=a_{j}\left(C_{i}-C_{j}\right) \beta_{j}+a_{l}\left(C_{i}-C_{l}\right) \beta_{l} \text { and } \lambda=(\tilde{c}-c)\left(a_{j}\left(C_{i}-C_{j}\right) \varphi_{j}+a_{l}\left(C_{i}-C_{l}\right) \varphi_{l}\right),
$$

with $C_{i}=-L_{i}^{-1}$ for $1 \leq i \leq k$. It is easily seen that this can not happen if $f$ satisfies the assumptions of either Proposition 1 or 2, depending on whether $c=\tilde{c}$ or $c \neq \tilde{c}$, respectively, if the codimension attains its minimum possible values $p=n$ and $p=n-1$, respectively. Therefore, in these cases the last assumption in Theorem 34 can be dropped.

\section{The $P$-transformation}

In this section we obtain reductions of the $L$-transformation that preserve the classes of $n$-dimensional flat Lagrangian submanifolds of $\mathbb{C}^{n}=\mathbb{R}^{2 n}$ and $n$-dimensional submanifolds 
with constant sectional curvature $c$ of $\mathbb{S}_{\epsilon}^{2 n+1}(c)$ that are horizontal with respect to the Hopf fibration $\pi: \mathbb{S}_{\epsilon}^{2 n+1}(c) \rightarrow \tilde{M}^{n}(4 c)$. We also obtain a further reduction that preserves the class of $n$-dimensional flat Lagrangian submanifolds of $\mathbb{R}^{2 n}$ that are contained in $\mathbb{S}^{2 n-1}$.

\subsection{The $P$-transformation for flat Lagrangian submanifolds}

An isometric immersion $f: M^{n} \rightarrow \tilde{M}^{n}$ of an $n$-dimensional Riemannian manifold into a Kaehler manifold of complex dimensional $n$ is said to be Lagrangian if the almost complex structure of $\tilde{M}^{m}$ carries each tangent space of $M^{n}$ onto its corresponding normal space.

For a Lagrangian isometric immersion $f: M^{n} \rightarrow \mathbb{C}^{n}$, comparing normal and tangential components of $\tilde{\nabla}_{X} J f_{*} Y=J \tilde{\nabla}_{X} f_{*} Y$ yields, respectively,

$$
\nabla_{X}^{\perp} J f_{*} Y=J f_{*} \nabla_{X} Y
$$

and

$$
-f_{*} A_{J f_{*} Y} X=J \alpha(X, Y)
$$

for all $X, Y \in \mathfrak{X}(M)$. It follows from (69) that

$$
R^{\perp}(X, Y) J f_{*} Z=J f_{*} R(X, Y) Z
$$

for all $X, Y, Z \in \mathfrak{X}(M)$. In particular, $M^{n}$ is flat if and only if $f$ has flat normal bundle.

Lagrangian isometric immersions $f: M^{n}(0) \rightarrow \mathbb{C}^{n}$ with $\nu_{f} \equiv 0$ have been characterized as follows in [5] among isometric immersions $f: M^{n}(0) \rightarrow \mathbb{C}^{n}$ with $\nu_{f} \equiv 0 \equiv R^{\perp}$.

Theorem 36. If $f: M^{n}(0) \rightarrow \mathbb{R}^{2 n} \cong \mathbb{C}^{n}$ is an isometric immersion with $\nu_{f} \equiv 0 \equiv R^{\perp}$ and $(v, h)$ is its associated solution of (2), then $f$ is Lagrangian if and only if $h=h^{t}$.

Corollary 37. Let $f: M^{n}(0) \rightarrow \mathbb{R}^{2 n}$ be a Lagrangian isometric immersion with $\nu_{f} \equiv 0$. Then there exist locally principal coordinates $u_{1}, \ldots, u_{n}$ on $M^{n}(0)$ and positive smooth functions $v_{1}, \ldots, v_{n}$ such that $d s^{2}=\sum_{j} v_{j}^{2} d u_{j}^{2}$ and $\alpha\left(\partial_{i}, \partial_{j}\right)=\delta_{i j} J \partial_{i}$, where $v=\left(v_{1}, \ldots, v_{n}\right)$ and $h=\left(h_{i j}\right), h_{i j}=v_{i}^{-1} \partial_{i} v_{j}$, satisfy the system of PDE's

$$
\left\{\begin{array}{l}
\text { i) } \left.\partial_{j} v_{i}=h_{j i} v_{j}, \quad 1 \leq i \neq j \leq n, \quad i i\right)\left(\sum_{\ell=1}^{n} \partial_{\ell}\right) h_{i j}=0 \\
i i i) \partial_{\ell} h_{i j}=h_{i \ell} h_{j \ell}, \quad 1 \leq \ell \neq i \neq j \neq \ell \leq n .
\end{array}\right.
$$

Conversely, if $(v, h)$ is a solution of (71) on an open simply connected subset $U \subset \mathbb{R}^{n}$ such that $v_{i} \neq 0$ everywhere then there exists a Lagrangian immersion $f: U \rightarrow \mathbb{C}^{n}$ with flat induced metric $d s^{2}=\sum_{i} v_{i}^{2} d u_{i}$ and $\nu_{f} \equiv 0$. 
Definition 38. Let $\tilde{f}=\mathcal{R}_{\varphi, \beta, \Omega}(f): \tilde{M}^{n} \rightarrow \mathbb{R}^{2 n}$ be a vectorial Ribaucour transform of a Lagrangian isometric immersion $f: M^{n}(0) \rightarrow \mathbb{R}^{2 n}$ determined by $(\varphi, \beta, \Omega)$, with $\varphi \in$ $\Gamma(V), \beta \in \Gamma\left(V^{*} \otimes N_{f} M\right)$ and $\Omega \in \Gamma\left(V^{*} \otimes V\right)$. If there exists $P \in V^{*} \otimes V$ satisfying $\sigma(P) \cap(-\sigma(P))=\emptyset$, where $\sigma(P)$ denotes the set of (complex) eigenvalues of $P$, such that

$$
\beta=J f_{*} \omega^{t} P
$$

where $\omega=d \varphi$, and

$$
\Omega^{t} P+P^{t} \Omega^{t}+T \rho=0
$$

where $T=-P^{t}-\left(P^{t}\right)^{-1}$ and $\rho=P^{t} \omega \omega^{t} P$, then $\tilde{f}$ is called a $P$-vectorial Ribaucour transform of $f$, or simply a P-transform of $f$. We write $\tilde{f}=\mathcal{R}_{\varphi, \beta, \Omega, P}(f)$.

Remark 39. As in the case of the $L$-transformation, if $\langle\cdot, \cdot\rangle \sim$ is another inner product on $V$, with $\langle\cdot, \cdot\rangle^{\sim}=\langle\cdot, B \cdot\rangle$ for some invertible $B \in V^{*} \otimes V$, then conditions (72) and (73) are satisfied by $(\varphi, \beta, \Omega, P,\langle\cdot, \cdot\rangle)$ if and only if they are satisfied by $\left(\hat{\varphi}, \hat{\beta}, \hat{\Omega}, \hat{P},\langle\cdot, \cdot\rangle^{\sim}\right)$, where $\hat{\varphi}=\varphi, \hat{\beta}=\beta B, \hat{\Omega}=\Omega B$ and $\hat{P}=B^{-1} P B$. Thus, in the definition of the $P$-transformation one may replace $(\varphi, \beta, \Omega, P,\langle\cdot, \cdot\rangle)$ by any quintuple $\left(\hat{\varphi}, \hat{\beta}, \hat{\Omega}, \hat{P},\langle\cdot, \cdot\rangle^{\sim}\right)$ related to $(\varphi, \beta, \Omega, P,\langle\cdot, \cdot\rangle)$ in this way. As before, the transposes of $\hat{P}$ and $P$ with respect to $\langle\cdot, \cdot\rangle \sim$ and $\langle\cdot, \cdot\rangle$ coincide, and if $P$ is diagonalizable then one can always assume that $P$ is symmetric after an appropriate change of the inner product on $V$.

Given endomorphisms $P, C \in V^{*} \otimes V$ of a Euclidean vector space $V$, the equation

$$
P^{t} X+X P=C
$$

for $X \in V^{*} \otimes V$ is known as the Lyapunov equation. Thus, if $\tilde{f}=\mathcal{R}_{\varphi, \beta, \Omega, P}(f)$ is a $P$ transform of $f$, then $\Omega$ satisfies a Lyapunov-type equation. The following result of [8] provides a sufficient condition on $P$ in order that (74) admit a unique solution $X$, and gives an explicit expression for $X$ as a polynomial on $P$ and $C$.

Theorem 40. If $P \in V^{*} \otimes V$ satisfies $\sigma(P) \cap(-\sigma(P))=\emptyset$, then (74) admits a unique solution for any $C \in V^{*} \otimes V$, given by

$$
X=\left(q_{-P}\left(P^{t}\right)\right)^{-1} \sum_{\ell=1}^{k} a_{\ell} \sum_{i=0}^{\ell-1}(-1)^{i}\left(P^{t}\right)^{\ell-1-i} C P^{i}
$$

where $q_{-P}(x)=\sum_{\ell=0}^{k} a_{\ell} x^{\ell}, a_{k}=1$, is the characteristic polynomial of $-P$.

In order to prove that the $P$-transformation preserves the class of $n$-dimensional flat Lagrangian submanifolds of $\mathbb{C}^{n}=\mathbb{R}^{2 n}$ we need the special case for $c=0$ of the following result. The case $c \neq 0$ will be used in our study in the next section of the $P$-transformation for $n$-dimensional submanifolds with constant sectional curvature $c$ of $\mathbb{S}_{\epsilon}^{2 n+1}(c)$ that are horizontal with respect to the Hopf fibration $\pi: \mathbb{S}_{\epsilon}^{2 n+1}(c) \rightarrow \tilde{M}^{n}(4 c)$. 
Proposition 41. Let $V$ and $W$ be Euclidean vector spaces, let $P \in V^{*} \otimes V$ be such that $\sigma(P) \cap(-\sigma(P))=\emptyset$, let $c \in \mathbb{R}, \psi \in V^{*}$ and $\nu \in V^{*} \otimes W$ and let $X \in V^{*} \otimes V$ be the unique solution of the Lyapunov equation

$$
X^{t} P+P^{t} X^{t}=-T \rho
$$

where $T=-P^{t}-\left(P^{t}\right)^{-1}$ and $\rho=P^{t} Q P$, with $Q=\nu^{t} \nu+c \psi^{t} \psi$. Then $X$ satisfies

$$
\begin{gathered}
X+X^{t}=Q+\rho, \\
T X-X^{t} T^{t}=0
\end{gathered}
$$

and

$$
X L+L^{t} X^{t}=\rho \text {, where } L=\left(P^{2}+I\right)^{-1} P^{2} .
$$

Proof: Since

$$
(Q+\rho) P+P^{t}(Q+\rho)+2 T \rho=(-Q P+\rho P)-(-Q P+\rho P)^{t}
$$

is a skew-symmetric matrix, the symmetric part of the unique solution $X$ of (76) must coincide with $(Q+\rho) / 2$. This gives (77)). Equation (78) follows easily from (76) and (77). Now observe that $T^{t}=-P-P^{-1}=-P^{-1}\left(I+P^{2}\right)$. Since $\sigma(P) \cap(-\sigma(P))=\emptyset$, it follows that $T$ is invertible and

$$
T^{t} L=-P \text {. }
$$

Therefore

$$
\begin{aligned}
T\left(X L+L^{t} X^{t}\right) & =T X L+T L^{t} X^{t}=X^{t} T^{t} L+T L^{t} X^{t} \\
& =-X^{t} P-P^{t} X^{t}=T \rho .
\end{aligned}
$$

Lemma 42. Any P-transform of a Lagrangian isometric immersion $f: M^{n}(0) \rightarrow \mathbb{R}^{2 n}$ is also an L-transform of $f$ with $L=\left(P^{2}+I\right)^{-1} P^{2}$.

Proof: It follows from (172) that $\nabla_{X} \beta=J f_{*}\left(\nabla_{X} \omega^{t}\right) P$ for all $X \in \mathfrak{X}(M)$, hence (15) becomes

$$
\alpha\left(X, \omega^{t}(v)\right)+J f_{*}\left(\left(\nabla_{X} \omega^{t}\right) P v\right)=0,
$$

which can also be writtten as

$$
d \omega^{t}(X) P=f_{*}^{t} J A(X)^{t} \omega^{t} .
$$

On the other hand, from (70) we obtain $f_{*}^{t} J A(X)^{t}=-A(X) J f_{*} Y$ for all $X \in \mathfrak{X}(M)$. Therefore

$$
A(X) \beta=A(X) J f_{*} \omega^{t} P=-f_{*}^{t} J A(X)^{t} \omega^{t} P=-d \omega^{t}(X) P^{2} .
$$


Thus, the tensor

$$
\Phi(X) v=\left(\nabla_{X} \omega^{t}\right) v-A_{\beta(v)} X=d \omega^{t}(X) v-A(X) \beta(v)
$$

satisfies

$$
\Phi(X)=d \omega^{t}(X)\left(P^{2}+I\right),
$$

and hence $\Phi(X) L+A(X) \beta=0$ for all $X \in \mathfrak{X}(M)$. Finally, that $\Omega$ satisfies (32) follows from Proposition 41 .

It follows from Lemma 42 and Theorem 13 that if $\tilde{f}=\mathcal{R}_{\varphi, \beta, \Omega, P}(f): \tilde{M}^{n} \rightarrow \mathbb{R}^{2 n}$ is a $P$-transform of a Lagrangian isometric immersion $f: M^{n}(0) \rightarrow \mathbb{R}^{2 n}$ then $\tilde{M}^{n}$ is also flat. We shall prove in Theorem 44 below that $\tilde{f}$ is also Lagrangian. First we express equation (80) in the local principal coordinates given by Corollary 37 .

Proposition 43. Let $f: M^{n}(0) \rightarrow \mathbb{R}^{2 n}$ be a Lagrangian isometric immersion with $\nu_{f} \equiv 0$, let $\left(u_{1}, \ldots, u_{n}\right)$ be principal coordinates given by Corollary 37 on an open subset $U \subset$ $M^{n}(0)$, let $(v, h)$ be the solution of (71) associated to $f$ and let $P$ be an invertible endomorphism of a Euclidean vector space $V$. If $\varphi: U \rightarrow V$ is such that $\omega=d \varphi$ satisfies (80), then $\left(\varphi, \gamma_{1}, \ldots, \gamma_{n}\right)$, with $\gamma_{i}=v_{i}^{-1} \omega\left(\partial_{i}\right)$ for $1 \leq i \leq n$, is a solution of

$$
\begin{cases}\text { i) } \partial_{i} \varphi=v_{i} \gamma_{i}, & \text { ii) } \partial_{i} \gamma_{i}=-\left(P^{t}\right)^{-1} \gamma_{i}-\sum_{j \neq i} h_{i j} \gamma_{j}, \\ \text { iii) } \partial_{i} \gamma_{j}=h_{i j} \gamma_{i} j \neq i . & \end{cases}
$$

Conversely, if $\left(\varphi, \gamma_{1}, \ldots, \gamma_{n}\right)$ is a solution of (82) then $\omega=d \varphi$ satisfies (80) and $\omega\left(\partial_{i}\right)=$ $v_{i} \gamma_{i}$ for $1 \leq i \leq n$.

Theorem 44. If $f: M^{n}(0) \rightarrow \mathbb{R}^{2 n}$ is a Lagrangian isometric immersion with $\nu_{f} \equiv 0$ then any P-transform $\tilde{f}=\mathcal{R}_{\varphi, \beta, \Omega, P}(f): \tilde{M}^{n}(0) \rightarrow \mathbb{R}^{2 n}$ of $f$ is also Lagrangian.

Proof: According to Theorem [36, it suffices to prove that the pair $(\tilde{v}, \tilde{h})$ associated to $\tilde{f}$ with respect to the local principal coordinates $u_{1}, \ldots, u_{n}$ given by Proposition 1 satisfies $\tilde{h}^{t}=\tilde{h}$. In view of (50), this is equivalent to

$$
\left\langle L^{-1} \Omega^{-1} \gamma_{i}, \beta^{j}\right\rangle=\left\langle\beta^{i}, L^{-1} \Omega^{-1} \gamma_{j}\right\rangle
$$

where $\gamma_{1}, \ldots, \gamma_{n}, \beta^{1}, \ldots, \beta^{n}$ are given by (33). By (78), we have

$$
T \Omega=\Omega^{t} T^{t}
$$

where $T=-P^{t}-\left(P^{t}\right)^{-1}$. From (33) and (72) we obtain

$$
P^{t} \gamma_{i}=P^{t} \omega\left(X_{i}\right)=\beta^{t} J f_{*} X_{i}=\beta^{t} \xi_{i}=\beta^{i}
$$


where $X_{i}=v_{i}^{-1} \partial_{i}, 1 \leq i \leq n$. We conclude from (779), (84) and (85) that (83) holds.

In order to prove the existence of $P$-transforms of any Lagrangian isometric immersion $f: M^{n}(0) \rightarrow \mathbb{R}^{2 n}$ with $\nu_{f} \equiv 0$, we need the special case $c=0$ of the folowing algebraic result, whose general form will be used in the next section.

Proposition 45. Let $V$ and $W$ be Euclidean vector spaces, let $P \in V^{*} \otimes V$ be such that $\sigma(P) \cap(-\sigma(P))=\emptyset$, let $\psi \in V^{*}, \nu \in V^{*} \otimes W, c \in \mathbb{R}$, and let $X=X(\nu, \psi, c)$ be the unique solution of (76).

(i) If $c \geq 0$, then $X$ is invertible if and only if $\operatorname{ker}\left(P^{c}-\alpha I\right) \cap Y^{c}=\{0\}$ for any eigenvalue $\alpha$ of $P^{c}$, where $Y^{c}$ is the complexified of

$$
Y=\left\{\begin{array}{l}
\operatorname{ker} \nu, \text { if } c=0 ; \\
\operatorname{ker} \psi \cap \operatorname{ker} \nu, \text { if } c \neq 0 .
\end{array}\right.
$$

(ii) If $c<0$ and $\operatorname{ker}\left(P^{c}-\alpha I\right) \cap(\operatorname{ker} \nu)^{c}=\{0\} \quad$ (resp., $\left.\operatorname{ker}\left(P^{c}-\alpha I\right) \cap(\operatorname{ker} \psi)^{c}=\{0\}\right)$ for any eigenvalue $\alpha$ of $P^{c}$, then there exists $\epsilon>0$ such that $X(\nu, \psi, c)$ is invertible if $|\psi|<\epsilon$ (resp., $|\nu|<\epsilon)$.

Proof: $(i)$ Assume first that $\operatorname{ker}\left(P^{c}-\alpha I\right) \cap Y^{c}=\{0\}$ for any eigenvalue $\alpha$ of $P^{c}$. If $u \in \operatorname{ker} X^{t}$, we obtain from (77) that

$$
0=|\nu u|^{2}+c(\psi u)^{2}+|\nu P u|^{2}+c(\psi P u)^{2} .
$$

Since $c \geq 0$, it follows that $u \in Y$. Thus ker $X^{t} \subset Y$, and (77) implies that $\operatorname{ker} X^{t}=\operatorname{ker} X$. Then (76) by $P(\operatorname{ker} X) \subset \operatorname{ker} X$, and since $\operatorname{ker} X \subset Y$, $\operatorname{ker} X=\{0\}$.

Conversely, if $u \in \operatorname{ker}\left(P^{c}-\alpha I\right) \cap Y^{c}$, then $X_{s} u=0$ and $P^{t} X_{a}(u)=-\alpha X_{a}(u)$ by (76) and (77). Since $\sigma(P) \cap(-\sigma(P))=\emptyset$, it follows that $X_{a}(u)=0$, hence $X(u)=0$. Thus $u=0$.

(ii) First observe that the unique solution $X(\nu, \psi, c)$ of (76) given by (75) depends continuously on $(\psi, \nu, c)$. Assume first that $\operatorname{ker}\left(P^{c}-\alpha I\right) \cap(\operatorname{ker} \nu)^{c}=\{0\}$. It follows from case $(i)$ for $c=0$ that $X(\nu, 0, c)$ is invertible. Thus, there exists $\epsilon>0$ such that $X(\nu, \psi, c)$ is invertible for all $\psi$ with $|\psi|<\epsilon$.

Suppose now that $\operatorname{ker}\left(P^{c}-\alpha I\right) \cap(\operatorname{ker} \psi)^{c}=\{0\}$ for any eigenvalue $\alpha$ of $P^{c}$. From part (i) for $c>0$, we have that $X(0, \psi, c)$ is invertible. Therefore, there exists $\epsilon>0$ such that $X(\nu, \psi, c)$ is invertible for all $\nu$ with $|\nu|<\epsilon$.

Theorem 46. Let $f: M^{n}(0) \rightarrow \mathbb{R}^{2 n}$ be a Lagrangian isometric immersion with $\nu_{f} \equiv 0$ and let $P$ an endomorphism of a Euclidean vector space $V$ such that $\sigma(P) \cap(-\sigma(P))=\emptyset$. Fixed $x_{0} \in M^{n}$, let $\varphi_{0} \in V$ and $\omega_{0} \in T_{x_{0}}^{*} M \otimes V$ be such that $\operatorname{ker}\left(P^{c}-\alpha I\right) \cap \operatorname{ker}\left(\omega_{0}^{t}\right)^{c}=\{0\}$ for any eigenvalue $\alpha$ of $P^{c}$. Then there exist an open neighborhood $U$ of $x_{0}$ and a unique $P$-transform $\tilde{f}=\mathcal{R}_{\varphi, \beta, \Omega, P}\left(\left.f\right|_{U}\right)$ of $\left.f\right|_{U}$ such that $\varphi\left(x_{0}\right)=\varphi^{0}$ and $d \varphi\left(x_{0}\right)=\omega_{0}$. 
Proof: Let $\left(u_{1}, \ldots, u_{n}\right)$ be principal coordinates given by Corollary 37 on an open simplyconnected neighborhood $U \subset M^{n}(0)$ of $x_{0}$ and let $(v, h)$ be the solution of (71) associated to $f$. It is easily checked that the compatibility conditions of system (82) are satisfied by virtue of (71). Therefore, if $\gamma_{i}^{0}=v_{i}^{-1}\left(x_{0}\right) \omega_{0}\left(\partial_{i}\left(x_{0}\right)\right)$ for $1 \leq i \leq n$, then there exists a unique solution $\left(\varphi, \gamma_{1}, \ldots, \gamma_{n}\right)$ of (82) such that $\varphi\left(x_{0}\right)=\varphi^{0}$ and $\gamma_{i}\left(x_{0}\right)=\gamma_{i}^{0}$ for all $1 \leq i \leq n$. By Proposition [43, $\omega=d \varphi$ satisfies (80) and $\omega\left(\partial_{i}\right)=v_{i} \gamma_{i}$ for $1 \leq i \leq n$.

Now, by Proposition 41 the Lyapunov equation $X^{t} P+P^{t} X^{t}=-T \rho_{0}$, where $T=$ $-P^{t}-\left(P^{t}\right)^{-1}$ and $\rho_{0}=P^{t} \omega_{0} \omega_{0}^{t} P$, has a unique solution $X=\Omega^{0}$, and $2 \Omega_{s}^{0}=\mathcal{F}^{t}\left(x_{0}\right) \mathcal{F}\left(x_{0}\right)$. Moreover, $\Omega^{0}$ is invertible by Proposition 45. Define $\beta \in \Gamma\left(V^{*} \otimes N_{f} M\right)$ by (72), and let $\Omega$ be the unique solution of (9) such that $\Omega\left(x_{0}\right)=\Omega_{0}$. Shrinking $U$ if necessary, we may assume that $\Omega$ is invertible on $U$. Using (81) we obtain

$$
\begin{aligned}
d\left(\Omega^{t} P+P^{t} \Omega^{t}+T \rho\right)(X)= & \Phi(X)^{t} \omega^{t} P+P^{t} \Phi(X)^{t} \omega^{t}-\left(P^{t}\right)^{2} d \omega(X) \omega^{t} P-d \omega(X) \omega^{t} P \\
& -\left(P^{t}\right)^{2} \omega d \omega^{t}(X) P-\omega d \omega^{t}(X) P=0 .
\end{aligned}
$$

Since (73) holds at $x_{0}$, it holds on $U$, hence $\tilde{f}=\mathcal{R}_{\varphi, \beta, \Omega}\left(\left.f\right|_{U}\right)$ is a $P$-transform of $\left.f\right|_{U}$.

In the following corollary we summarize the process given by the $P$-transformation to generate new Lagrangian isometric immersions $f: M^{n}(0) \rightarrow \mathbb{R}^{2 n}$ starting with a given one and a vector-valued solution of the linear system of PDE's (82).

Corollary 47. Let $f: M^{n}(0) \rightarrow \mathbb{R}^{2 n}$ be a Lagrangian isometric immersion with $\nu_{f} \equiv 0$, let $(v, h)$ be the solution of (71) associated to $f$ on an open subset $U \subset M^{n}(0)$ endowed with principal coordinates $\left(u_{1}, \ldots, u_{n}\right)$ given by Corollary 37 , let $P$ be an endomorphism of a Euclidean vector space $V$ such that $\sigma(P) \cap(-\sigma(P))=\emptyset$, and let $\left(\varphi, \gamma_{1}, \ldots, \gamma_{n}\right)$ be a $V$-valued solution of the linear system of PDE's (82) on an open subset $W \subset U$ where

$$
\tilde{v}_{i}=v_{i}+\left\langle\gamma_{i},\left(P+P^{-1}\right) \Omega^{-1} \varphi\right\rangle
$$

does not vanish for all $1 \leq i \leq n$, with $\Omega^{t}$ given by 75) for $C=\left(\left(P^{t}\right)^{2}+I\right) d \varphi(d \varphi)^{t} P$. Then $\tilde{f}: W \rightarrow \mathbb{R}^{2 n}=\mathbb{C}^{n}$ given by

$$
\tilde{f}=f-\sum_{j=1}^{n}\left(\left\langle\Omega^{-1} \varphi, \gamma_{j}\right\rangle+\left\langle P \Omega^{-1} \varphi, \gamma_{j}\right\rangle i\right) f_{*} X_{j},
$$

where $X_{j}=v_{j}^{-1} \partial_{j}$ for $1 \leq j \leq n$, defines a new Lagrangian isometric immersion with flat induced metric. Moreover, the pair associated to $\tilde{f}$ is $(\tilde{v}, \tilde{h})$, where

$$
\tilde{h}_{i j}=h_{i j}+\left\langle\gamma_{j},\left(P+P^{-1}\right) \Omega^{-1} \gamma_{i}\right\rangle .
$$

In particular, $(\tilde{v}, \tilde{h})$ is a new solution of (71). 


\subsection{The $P^{*}$-transformation}

Lagrangian isometric immersions $f: M^{n}(0) \rightarrow \mathbb{C}^{n}$ satisfying $f\left(M^{n}(0)\right) \subset \mathbb{S}^{2 n-1}$ are of special interest in view of the following result of [3].

Theorem 48. An isometric immersion $f: M^{n}(0) \rightarrow \mathbb{R}^{2 n}$ is Lagrangian and satisfies $f\left(M^{n}(0)\right) \subset \mathbb{S}^{2 n-1}$ if and only if it is the lifting by the Hopf projection $\pi: \mathbb{S}^{2 n-1} \rightarrow \mathbb{C P}^{n-1}$ of a Lagrangian isometric immersion $F: M^{n-1}(0) \rightarrow \mathbb{C P}^{n-1}$.

We will need the following characterization obtained in [4] of isometric immersions $f: M^{n}(0) \rightarrow \mathbb{R}^{2 n}$ with flat normal bundle and $\nu_{f} \equiv 0$ that satisfy $f\left(M^{n}(0)\right) \subset \mathbb{S}^{2 n-1}$.

Corollary 49. Let $f: M^{n}(0) \rightarrow \mathbb{R}^{2 n}$ be an isometric immersion with $\nu_{f} \equiv 0 \equiv R^{\perp}$, and let $(v, h)$ be its associated pair with respect to the local principal coordinates $\left(u_{1}, \ldots, u_{n}\right)$ on $M^{n}(0)$ given by Proposition 1. Then $f\left(M^{n}(0)\right) \subset \mathbb{S}^{2 n-1}$ if and only if $\sum_{i=1}^{n} v_{i}^{2}=1$.

We now look for the $P$-transformations that preserve the class of Lagrangian isometric immersions $f: M^{n}(0) \rightarrow \mathbb{R}^{2 n}$ such that $f\left(M^{n}(0)\right) \subset \mathbb{S}^{2 n-1}$.

Lemma 50. Let $f: M^{n}(0) \rightarrow \mathbb{R}^{2 n}$ be a Lagrangian isometric immersion such that $f\left(M^{n}(0)\right) \subset \mathbb{S}^{2 n-1} \subset \mathbb{R}^{2 n}$ and let $(v, h)$ be its associated solution of (71) on an open subset $U \subset M^{n}(0)$ endowed with principal coordinates given by Corollary 37 . If $\tilde{f}=\mathcal{R}_{\varphi, \beta, \omega, P}(f)$ is a P-transform of $f$ and $\gamma_{i}=v_{i}^{-1} \omega\left(\partial_{i}\right), 1 \leq i \leq n$, then $\varphi+\sum_{i} v_{i} P^{t} \gamma_{i}$ is constant on $U$.

Proof: Using (82) and $\sum_{i=1}^{n} v_{i}^{2}=1$ we obtain

$$
\begin{aligned}
\partial_{i}\left(\varphi+\sum_{j=1}^{n} v_{j} P^{t} \gamma_{j}\right) & =v_{i} \gamma_{i}+P^{t} \sum_{i \neq j}\left(v_{j} \partial_{i} \gamma_{j}+\gamma_{j} \partial_{i} v_{j}\right)+v_{i} P^{t} \partial_{i} \gamma_{i}+\partial_{i} v_{i} P^{t} \gamma_{i} \\
= & v_{i} \gamma_{i}+P^{t} \sum_{i \neq j}\left(h_{i j} \gamma_{i} v_{j}+\gamma_{j} h_{i j} v_{i}\right)-P^{t}\left(P^{t}\right)^{-1} \gamma_{i} v_{i} \\
& -P^{t} \sum_{i \neq j} \gamma_{j} h_{i j} v_{i}-P^{t} \gamma_{i} \sum_{j \neq i} h_{i j} v_{j}=0 .
\end{aligned}
$$

Definition 51. Let $f: M^{n}(0) \rightarrow \mathbb{R}^{2 n}$ be a Lagrangian isometric immersion such that $f\left(M^{n}(0)\right) \subset \mathbb{S}^{2 n-1} \subset \mathbb{R}^{2 n}$ and let $(v, h)$ be its associated solution of (71) on an open subset $U \subset M^{n}(0)$ endowed with principal coordinates given by Corollary 37. A $P$-transform $\tilde{f}=\mathcal{R}_{\varphi, \beta, \Omega, P}(f)$ of $\left.f\right|_{U}$ is said to be a $P^{*}$-transform of $\left.f\right|_{U}$ if

$$
\varphi+\sum_{i=1}^{n} v_{i} P^{t} \gamma_{i}=0
$$

Remark 52. Notice that (87) is satisfied by $(\varphi, \beta, \Omega, P,\langle\cdot, \cdot\rangle)$ if and only if it is satisfied by $\left(\hat{\varphi}, \hat{\beta}, \hat{\Omega}, \hat{P},\langle\cdot, \cdot\rangle^{\sim}\right)$, where $\langle\cdot, \cdot\rangle^{\sim}=\langle\cdot, B \cdot\rangle$ for some invertible $B \in V^{*} \otimes V, \hat{\varphi}=\varphi$, $\hat{\beta}=\beta B, \hat{\Omega}=\Omega B$ and $\hat{P}=B^{-1} P B$, for the transposes of $\hat{P}$ and $P$ with respect to $\langle\cdot, \cdot\rangle^{\sim}$ and $\langle\cdot, \cdot\rangle$ coincide. 
Theorem 53. Let $f: M^{n}(0) \rightarrow \mathbb{R}^{2 n}$ be a Lagrangian isometric immersion such that $f\left(M^{n}(0)\right) \subset \mathbb{S}^{2 n-1}$. If $\tilde{f}=\mathcal{R}_{\varphi, \beta, \Omega, P}(f): \tilde{M}^{n}(0) \rightarrow \mathbb{R}^{2 n}$ is a $P^{*}$-transform of $f$, then $\tilde{f}\left(\tilde{M}^{n}(0)\right) \subset \mathbb{S}^{2 n-1}$.

Proof: Since $\sum_{i=1}^{n} v_{i}^{2}=1$, then $\tilde{v}$ given by (86) also satisfies $\sum_{i=1}^{n} \tilde{v}_{i}^{2}=1$ if and only if

$$
\begin{aligned}
0 & =\sum_{i=1}^{n} 2 v_{i}\left\langle\gamma_{i}, T^{t} \Omega^{-1} \varphi\right\rangle+\sum_{i=1}^{n}\left\langle\gamma_{i}, T^{t} \Omega^{-1} \varphi\right\rangle^{2} \\
& =-\sum_{i=1}^{n} 2 v_{i}\left\langle\omega^{t}\left(T^{t} \Omega^{-1} \varphi\right), X_{i}\right\rangle+\left\langle\omega^{t}\left(T^{t} \Omega^{-1} \varphi\right), \omega^{t}\left(T^{t} \Omega^{-1} \varphi\right)\right\rangle \\
& =\left\langle T^{t} \Omega^{-1} \varphi,-2 \sum_{i=1}^{n} v_{i} \gamma_{i}\right\rangle+\left\langle\varphi,\left(\Omega^{-1}\right)^{t} T \omega \omega^{t} T^{t} \Omega^{-1} \varphi\right) .
\end{aligned}
$$

That this is indeed the case follows from the fact that, by (73) and (87), the last expression is equal to

$$
\begin{aligned}
\left\langle T^{t} \Omega^{-1} \varphi, 2\left(P^{t}\right)^{-1} \varphi\right\rangle & +\left\langle\varphi,\left(\Omega^{-1}\right)^{t}\left(-\left(P^{t}\right)^{-1} \Omega^{t}-\Omega^{t} P^{-1}\right) T^{t} \Omega^{-1} \varphi\right\rangle \\
& =\left\langle T^{t} \Omega^{-1} \varphi, 2\left(P^{t}\right)^{-1} \varphi\right\rangle-\left\langle\varphi,\left(\Omega^{-1}\right)^{t}\left(P^{t}\right)^{-1} T \varphi\right\rangle-\left\langle\varphi, P^{-1} T^{t} \Omega^{-1} \varphi\right\rangle
\end{aligned}
$$

which vanishes by (84).

Corollary 54. Let $f: M^{n}(0) \rightarrow \mathbb{R}^{2 n}$ be a Lagrangian isometric immersion satisfying $f\left(M^{n}(0)\right) \subset \mathbb{S}^{2 n-1}$, let $(v, h)$ be its associated solution of (71), with $\sum_{i=1}^{n} v_{i}^{2}=1$, on an open subset $U \subset M^{n}(0)$ endowed with principal coordinates given by Corollary 37, and let $P$ be an endomorphism of a Euclidean vector space $V$ such that $\sigma(P) \cap(-\sigma(P))=\emptyset$. If $\left(\gamma_{1}, \ldots, \gamma_{n}\right)$ satisfies equations (ii) and (iii) of (82) on an open subset $W \subset U$ where

$$
\tilde{v}_{i}=v_{i}-\sum_{j=1}^{n} v_{j}\left\langle\Omega^{-1} \gamma_{i},\left(\left(P^{t}\right)^{2}+I\right) \gamma_{j}\right\rangle
$$

does not vanish for $1 \leq i \leq n$, with $\Omega^{t}$ given by 175$)$ for $C=\left(\left(P^{t}\right)^{2}+I\right) \sum_{i=1}^{n} \gamma_{i} \gamma_{i}^{t} P$, then $\tilde{f}: W \rightarrow \mathbb{R}^{2 n}$ given by

$$
\tilde{f}=f+\sum_{\ell, j=1}^{n}\left(\left\langle\Omega^{-1} P^{t} \gamma_{\ell}, \gamma_{j}\right\rangle+\left\langle\Omega^{-1} P^{t} \gamma_{\ell}, P^{t} \gamma_{j}\right\rangle i\right) v_{\ell} f_{*} X_{j}
$$

where $X_{j}=v_{j}^{-1} \partial_{j}$ for $1 \leq j \leq n$, defines a new Lagrangian isometric immersion with flat induced metric such that $\tilde{f}(W) \subset \mathbb{S}^{2 n-1}$. Moreover, $(\tilde{v}, \tilde{h})$ is the solution of (71) associated to $\tilde{f}$, with

$$
\tilde{h}_{i j}=h_{i j}+\left\langle\gamma_{j},\left(P+P^{-1}\right) \Omega^{-1} \gamma_{i}\right\rangle .
$$




\subsection{The $P$-transformation for horizontal submanifolds.}

Let $\mathbb{C}_{\epsilon}^{n+1}$ denote the complex $(n+1)$-space endowed with the pseudo-Euclidean metric

$$
g_{\epsilon}=\epsilon d z_{1} d \bar{z}_{1}+\sum_{j=2}^{n+1} d z_{j} d \bar{z}_{j}, \epsilon= \pm 1
$$

and let

$$
\mathbb{S}_{\epsilon}^{2 n+1}(c)=\left\{z \in \mathbb{C}_{\epsilon}^{n+1}: g_{\epsilon}(z, z)=\frac{1}{c}, \epsilon c>0\right\}
$$

stand for either the Euclidean sphere or the anti-de-Sitter space time of dimension $(2 n+1)$ and constant sectional curvature $c$, depending on whether $\epsilon=1$ or $\epsilon=-1$, respectively.

The complex numbers act on $\mathbb{C}_{\epsilon}^{n+1}$ by $\lambda\left(z_{1}, \ldots, z_{n+1}\right) \rightarrow\left(\lambda z_{1}, \ldots, \lambda z_{n+1}\right)$. The quotient space $\tilde{M}^{n}(4 c)$ of $\mathbb{S}^{2 n+1}(c)$ under the identification induced by this action is the complex projective space $\mathbb{C} \mathbb{P}^{n}(4 c)$ or complex hyperbolic space $\mathbb{C H}^{n}(4 c)$ of complex dimension $n$ and constant holomorphic curvature $4 c$, corresponding to $c>0$ or $c<0$, respectively. If $\pi: \mathbb{S}_{\epsilon}^{2 n+1} \rightarrow \tilde{M}^{n}(4 c)$ is the quotient map, $\tilde{J}$ is the complex structure on $\mathbb{C}_{\epsilon}^{n+1}$ defined by multiplication by $i$ and $\phi$ is its projection onto the tangent bundle of $\mathbb{S}^{2 n+1}(c)$, then the complex structure $J$ on $\tilde{M}^{n}(4 c)$ is given by $J \circ \pi_{*}=\pi_{*} \circ \phi$.

An isometric immersion $f: M \rightarrow \mathbb{S}_{\epsilon}^{2 n+1}(c) \subset \mathbb{C}_{\epsilon}^{n+1}$ of a Riemannian manifold is said to be horizontal if the structure vector field $\xi_{f}=\sqrt{|c|} \tilde{J} f$ is everywhere normal to $f$. If $f: M \rightarrow \mathbb{S}_{\epsilon}^{2 n+1}(c)$ is horizontal, then $f$ is anti-invariant with respect to $\phi$, that is, $\phi f_{*} T_{x} M \subset N_{f} M(x)$ for all $x \in M$. Moreover, the second fundamental form of $f$ satisfies

$$
A_{\xi_{f}}=0 \text { and } \phi \alpha(X, Y)=-f_{*} A_{\phi f_{*} Y} X
$$

for all $X, Y \in \mathfrak{X}(M)$, and the following relations hold:

$$
\begin{aligned}
\nabla_{X}^{\perp} \xi_{f} & =\sqrt{|c|} \phi f_{*} X \\
\phi^{2} X & =-X+\epsilon\left\langle X, \xi_{f}\right\rangle \xi_{f} \\
\langle\phi X, \phi Y\rangle & =\langle X, Y\rangle+\epsilon\left\langle X, \xi_{f}\right\rangle\left\langle Y, \xi_{f}\right\rangle \\
\nabla_{X}^{\perp} \phi f_{*} Y & =\phi f_{*} \nabla_{X} Y-\epsilon \sqrt{|c|}\langle X, Y\rangle \xi_{f} \\
R^{\perp}(X, Y) \xi_{f} & =0 \\
\left\langle R^{\perp}(X, Y) \phi f_{*} Z, \phi f_{*} W\right\rangle & =\langle R(X, Y) Z, W\rangle-c(\langle X, W\rangle\langle Y, Z\rangle-\langle X, Z\rangle\langle Y, W\rangle)
\end{aligned}
$$

In particular, a horizontal isometric immersion $f: M^{n} \rightarrow \mathbb{S}_{\epsilon}^{2 n+1}(c)$ has flat normal bundle if and only if $M^{n}$ has constant sectional curvature $c$.

The next result relates Lagrangian isometric immersions $f: M \rightarrow \tilde{M}^{n}(4 c)$ to horizontal isometric immersions $f: M \rightarrow \mathbb{S}_{\epsilon}^{2 n+1}(c)$. We refer to [13] for a proof. 
Theorem 55. If $f: M^{n} \rightarrow \mathbb{S}_{\epsilon}^{2 n+1}(c)$ is horizontal then $g=\pi \circ f$ is Lagrangian. Conversely, if $g: M \rightarrow \tilde{M}^{n}(4 c)$ is a Lagrangian isometric immersions and $\left(x_{0}, y_{0}\right) \in M \times$ $\mathbb{S}_{\epsilon}^{2 n+1}(c)$ is such that $g\left(x_{0}\right)=\pi\left(y_{0}\right)$, then there exist a Riemannian manifold $\hat{M}$, an isometric covering map $\tau: \hat{M} \rightarrow M$, a horizontal isometric immersion $\hat{f}: \hat{M} \rightarrow \mathbb{S}_{\epsilon}^{2 n+1}(c)$ and $\hat{x} \in \hat{M}$ such that $\pi \circ \hat{f}=g \circ \tau, \tau(\hat{x})=x_{0}$ and $\hat{f}(\hat{x})=y_{0}$.

It will be convenient to have Proposition 1 explicitly restated in this particular case.

Corollary 56. Let $f: M^{n}(c) \rightarrow \mathbb{S}_{\epsilon}^{2 n+1}(c)$ be an isometric immersion with flat normal bundle, $\nu_{f} \equiv 0$, and Riemannian first normal bundle when $c<0$. Then there exist local principal coordinates $\left(u_{1}, \ldots, u_{n}\right)$ on $M^{n}(c)$, a smooth orthonormal normal frame $\xi_{1}, \ldots, \xi_{n+1}$ and smooth functions $v_{1}, \ldots, v_{n}$ and $\rho_{1}, \ldots, \rho_{n}$, with $v_{i}>0$ for $1 \leq i \leq n$, such that

$$
d s^{2}=\sum_{i} v_{i}^{2} d u_{i}^{2}, \quad \alpha\left(\partial_{i}, \partial_{j}\right)=v_{i} \delta_{i j} \xi_{i}
$$

and

$$
\nabla_{\partial_{i}} X_{j}=h_{j i} X_{i}, \quad \nabla_{\partial_{i}}^{\perp} \xi_{j}=h_{i j} \xi_{i}, i \neq j, \quad \nabla_{\partial_{i}}^{\perp} \xi_{n+1}=\rho_{i} \xi_{i},
$$

where $X_{i}=\left(1 / v_{i}\right) \partial_{i}$ and $h_{i j}=\left(1 / v_{i}\right) \partial_{i} v_{j}$ for $i \neq j$. Moreover, the triple $(v, h, \rho)$, where $v=\left(v_{1}, \ldots, v_{n}\right), h=\left(h_{i j}\right)$ and $\rho=\left(\rho_{1}, \ldots, \rho_{n}\right)$, satisfies the system of PDEs

$$
\left\{\begin{array}{l}
\text { i) } \left.\left.\partial_{j} v_{i}=h_{j i} v_{j}, \quad i i\right) \partial_{j} h_{i \ell}=h_{i j} h_{j \ell}, \quad i i i\right) \partial_{j} \rho_{i}=h_{i j} \rho_{j} \\
i v) \partial_{i} h_{i j}+\partial_{j} h_{j i}+\sum_{\ell=1}^{n} h_{\ell i} h_{\ell j}+c v_{i} v_{j}=0, \\
\text { v) } \partial_{j} h_{i j}+\partial_{i} h_{j i}+\sum_{\ell=1}^{n} h_{i \ell} h_{j \ell}+\epsilon \rho_{i} \rho_{j}=0, \epsilon=\frac{c}{|c|}, \quad i \neq j \neq \ell \neq i .
\end{array}\right.
$$

Conversely, if $(v, h, \rho)$ is a solution of (95) on an open simply connected subset $U \subset$ $\mathbb{R}^{n}$ such that $v_{i}>0$ everywhere, then there exists an immersion $f: U \rightarrow \mathbb{S}_{\epsilon}^{2 n+1}(c)$ with flat normal bundle, $\nu_{f} \equiv 0$, Riemannian first normal bundle and induced metric $d s^{2}=\sum_{i} v_{i}^{2} d u_{i}^{2}$ of constant sectional curvature $c$.

We shall use the following results proved in [16].

Theorem 57. An isometric immersion $f: M^{n}(c) \rightarrow \mathbb{S}_{\epsilon}^{2 n+1}(c)$ as in Corollary 56 is horizontal if and only if its associated triple $(v, h, \rho)$ satisfies

$$
h_{i j}=h_{j i} \text { and } \rho_{i}=\sqrt{|c|} v_{i} \text {. }
$$

Corollary 58. Let $f: M^{n}(c) \rightarrow \mathbb{S}_{\epsilon}^{2 n+1}(c)$ be a horizontal isometric immersion with $\nu_{f} \equiv 0$. Then there exist locally principal coordinates $\left(u_{1}, \ldots, u_{n}\right)$ on $M^{n}(c)$ such that

$$
d s^{2}=\sum_{i} v_{i}^{2} d u_{i}^{2}, v_{i}>0 \text { and } \alpha\left(\partial_{i}, \partial_{j}\right)=\delta_{i j} \phi \partial_{i},
$$


where $v=\left(v_{1}, \ldots, v_{n}\right)$ and $h=\left(h_{i j}\right)$, with $h_{i j}=h_{j i}$ for $1 \leq i \neq j \leq n$, satisfy the system of PDE's

$$
\left\{\begin{array}{l}
\text { i) } \left.\partial_{j} v_{i}=h_{j i} v_{j}, \quad 1 \leq i \neq j \leq n, \quad i i\right)\left(\sum_{\ell=1}^{n} \partial_{\ell}\right) h_{i j}+c v_{i} v_{j}=0 \\
i i i) \partial_{\ell} h_{i j}=h_{i \ell} h_{j \ell}, \quad 1 \leq \ell \neq i \neq j \neq \ell \leq n .
\end{array}\right.
$$

Conversely, if $(v, h)$ is a solution of (97) on an open simply connected subset $U \subset \mathbb{R}^{n}$ such that $v_{i} \neq 0$ for $1 \leq i \leq n$ everywhere, then there exists a horizontal isometric immersion $f: U \rightarrow \mathbb{S}_{\epsilon}^{2 n+1}(c)$ with induced metric $d s^{2}=\sum v_{i}^{2} d u_{i}$ of constant sectional curvature $c$.

Definition 59. Let $\tilde{f}=\mathcal{R}_{\varphi, \beta, \Omega}(f): \tilde{M}^{n} \rightarrow \mathbb{S}_{\epsilon}^{2 n+1}(c)$ be a vectorial Ribaucour transform of a horizontal isometric immersion $f: M^{n}(c) \rightarrow \mathbb{S}_{\epsilon}^{2 n+1}(c)$ determined by $(\varphi, \beta, \Omega)$, with $\varphi \in \Gamma(V), \beta \in \Gamma\left(V^{*} \otimes N_{f} M\right)$ and $\Omega \in \Gamma\left(V^{*} \otimes V\right)$. If there exists $P \in V^{*} \otimes V$ satisfying $\sigma(P) \cap(-\sigma(P))=\emptyset$ such that

$$
\beta=\left(\phi f_{*} \omega^{t}+\epsilon \sqrt{|c|} \xi_{f} \varphi^{t}\right) P
$$

where $\omega=d \varphi$, and

$$
\Omega^{t} P+P^{t} \Omega^{t}+T \rho=0
$$

where $T=-P^{t}-\left(P^{t}\right)^{-1}$ and $\rho=P^{t} \omega \omega^{t} P+c P^{t} \varphi \varphi^{t} P=\beta^{t} \beta$, then $\tilde{f}$ is said to be a Ribaucour $P$-transform of $f$, or simply a $P$-transform of $f$. We write $\tilde{f}=\mathcal{R}_{\varphi, \beta, \Omega, P}(f)$.

Lemma 60. Any P-transform of a horizontal isometric immersion $f: M^{n}(c) \rightarrow \mathbb{S}_{\epsilon}^{2 n+1}(c)$ is also an $L$-transform of $f$ with $L=\left(P^{2}+I\right)^{-1} P^{2}$.

Proof: It follows from (93) and (98) that

$$
\nabla_{X} \beta=\phi f_{*}\left(\nabla_{X} \omega^{t}\right) P+\epsilon \sqrt{|c|} \nabla_{X}^{\perp} \xi_{f} \varphi^{t} P
$$

for all $X \in \mathfrak{X}(M)$, hence (5) becomes

$$
\alpha\left(X, \omega^{t}(v)\right)+\phi f_{*}\left(\nabla_{X} \omega^{t}\right) P v+\epsilon \sqrt{|c|} \nabla_{X}^{\perp} \xi_{f} \varphi^{t} P v=0,
$$

which by (90) and (92) can also be writtten as

$$
d \omega^{t}(X) P=-f_{*}^{t} \phi^{t} A(X)^{t} \omega^{t}-c X \varphi^{t} P .
$$

On the other hand, from (89), (91) and (92) we obtain

$$
f_{*}^{t} \phi^{t} A(X)^{t}=A(X) \phi f_{*}
$$


for all $X \in \mathfrak{X}(M)$. Therefore, by (9) , (101) and (102),

$$
\begin{aligned}
A(X) \beta & =A(X)\left(\phi f_{*} \omega^{t}+\epsilon \sqrt{|c|} \xi_{f} \varphi^{t}\right) P \\
& =f_{*}^{t} \phi^{t} A(X)^{t} \omega^{t} P \\
& =-d \omega^{t}(X) P^{2}-c X \varphi^{t} P^{2} .
\end{aligned}
$$

Thus the tensor $\Phi(X)=d \omega^{t}(X)-A(X) \beta+c X \varphi^{t}$ satisfies

$$
\Phi(X)=\left(d \omega^{t}(X)+c X \varphi^{t}\right)\left(P^{2}+I\right),
$$

and hence $\Phi(X) L+A(X) \beta=0$ for all $X \in \mathfrak{X}(M)$. Finally, that $\Omega$ satisfies (32) follows from Proposition 41.

It follows from Theorem 13 and Lemma 60 that if $\tilde{f}=\mathcal{R}_{\varphi, \beta, \Omega, P}(f): \tilde{M}^{n} \rightarrow \mathbb{S}_{\epsilon}^{2 n+1}(c)$ is a $P$-transform of a horizontal isometric immersion $f: M^{n}(c) \rightarrow \mathbb{S}_{\epsilon}^{2 n+1}(c)$ then $\tilde{M}^{n}$ also has constant sectional curvature $c$. We shall prove that $\tilde{f}$ is also horizontal. For that we first express equation (100) in the local principal coordinates given by Corollary [58.

Proposition 61. Let $f: M^{n}(c) \rightarrow \mathbb{S}_{\epsilon}^{2 n+1}(c)$ be a horizontal isometric immersion with $\nu_{f} \equiv 0$, let $(v, h)$ be its associated solution of (97) with respect to principal coordinates given by Corollary 58, and let $P$ be an invertible endomorphism of a Euclidean vector space $V$. If $(\varphi, \omega=d \varphi)$ satisfies (100), then $\left(\varphi, \gamma_{1}, \ldots, \gamma_{n}\right)$, with $\gamma_{i}=v_{i}^{-1} \omega\left(\partial_{i}\right)$ for $1 \leq i \leq n$, is a solution of

$$
\left\{\begin{array}{l}
\text { i) } \left.\partial_{i} \varphi=v_{i} \gamma_{i}, \quad i i\right) \partial_{i} \gamma_{i}=-\left(P^{t}\right)^{-1} \gamma_{i}-\sum_{j \neq i} \gamma_{j} h_{i j}-c v_{i} \varphi \\
i i i) \partial_{i} \gamma_{j}=h_{i j} \gamma_{i} j \neq i,
\end{array}\right.
$$

Conversely, if $\left(\varphi, \gamma_{1}, \ldots, \gamma_{n}\right)$ is a solution of 104) then $(\varphi, \omega=d \varphi)$ satisfies (100) and $\omega\left(\partial_{i}\right)=v_{i} \gamma_{i}$ for $1 \leq i \leq n$.

Theorem 62. If $f: M_{\tilde{f}}^{n}(c) \rightarrow \mathbb{S}_{\epsilon}^{2 n+1}(c)$ is a horizontal isometric immersion with $\nu_{f} \equiv 0$ then any $P$-transform $\tilde{f}=\mathcal{R}_{\varphi, \beta, \Omega, P}(f): \tilde{M}^{n}(c) \rightarrow \mathbb{S}_{\epsilon}^{2 n+1}(c)$ of $f$ is also horizontal.

Proof: By Theorem [57, it suffices to prove that the triple $(\tilde{v}, \tilde{h}, \tilde{\rho})$ associated to $\tilde{f}$ satisfies (96). We claim that such triple is given by

$$
\tilde{v}_{i}=v_{i}-\left\langle\gamma_{i}, T^{t} \Omega^{-1} \varphi\right\rangle, \tilde{h}_{i j}=h_{i j}-\left\langle\gamma_{j}, T^{t} \Omega^{-1} \gamma_{i}\right\rangle \text { and } \tilde{\rho}_{i}=\rho_{i}-\sqrt{|c|}\left\langle T \varphi, \Omega^{-1} \gamma_{i}\right\rangle \text {, }
$$

where $T=-P^{t}-\left(P^{t}\right)^{-1}$. In fact, using (92) and the fact that $\xi_{i}=\phi f_{*} X_{i}, 1 \leq i \leq n$, we obtain from (79) and (98) that

$$
\beta^{i}=\beta^{t} \xi_{i}=P^{t} \omega f_{*}^{t} \phi^{t} \xi_{i}=P^{t} \omega f_{*}^{t} \phi^{t} \phi f_{*} X_{i}=P^{t} \omega\left(X_{i}\right)=P^{t} \gamma_{i}=-L^{t} T \gamma_{i}
$$


where $L=\left(P^{2}+I\right)^{-1} P^{2}$, and $\beta^{n+1}=\beta^{t}\left(\xi_{f}\right)=\sqrt{|c|} P^{t} \varphi$.

Thus equations (50) for $1 \leq r \leq n$ reduce to the first two equations in (105). Now, we have $\tilde{\xi}_{f}=\mathcal{P}\left(\xi_{f}+\sqrt{|c|} \sum_{r}\left\langle T \varphi, \Omega^{-1} \beta_{r}\right\rangle \xi_{r}\right)$. Using (8), (9), (18), (34), (94), (103) and (104), the last of equations (105) follows from

$$
\tilde{\nabla}_{\partial_{i}}^{\perp} \tilde{\xi}_{f}=\tilde{\rho}_{i} \mathcal{P}\left(\xi_{i}-\sum_{r}\left\langle\beta^{i}, L^{-1} \Omega^{-1} \beta^{r}\right\rangle \xi_{r}\right)=\tilde{\rho}_{i} \tilde{\xi}_{i}
$$

Since $T \Omega$ is symmetric by Theorem 41, it follows that $(\tilde{v}, \tilde{h}, \tilde{\rho})$ satisfies (96)).

We now prove the existence of $P$-transforms of any horizontal isometric immersion $f: M^{n}(c) \rightarrow \mathbb{S}^{2 n+1}(c)$ with $\nu_{f} \equiv 0$ satisfying suitable initial conditions.

Proposition 63. Let $f: M^{n}(c) \rightarrow \mathbb{S}^{2 n+1}(c)$ be a horizontal isometric immersion with $\nu_{f} \equiv 0$ and let $P$ be an endomorphism of a Euclidean vector space $V$ such that $\sigma(P) \cap$ $(-\sigma(P))=\emptyset$. Fixed $x_{0} \in M^{n}$ and $\varphi_{0} \in V, \omega_{0} \in T_{x_{0}} M^{*} \otimes V$ such that $\operatorname{ker}\left(P^{c}-\alpha I\right) \cap$ $\operatorname{ker}\left(\varphi_{0}^{t}\right)^{c} \cap \operatorname{ker}\left(\omega_{0}^{t}\right)^{c}=\{0\}$, there exist an open neighborhood $U$ of $x_{0}$ and a unique $P$ transform $\tilde{f}=\mathcal{R}_{\varphi, \beta, \Omega, P}\left(\left.f\right|_{U}\right)$ of $\left.f\right|_{U}$ such that $\varphi\left(x_{0}\right)=\varphi_{0}$ and $d \varphi\left(x_{0}\right)=\omega_{0}$.

Proof: Let $\left(u_{1}, \ldots, u_{n}\right)$ be principal coordinates given by Corollary 58 on an open simplyconnected neighborhood $U \subset M^{n}(c)$ of $x_{0}$ and let $(v, h)$ be the solution of (97) associated to $f$. It is easily checked that the compatibility conditions of (104) are satisfied by virtue of (97). Therefore, if $\gamma_{i}^{0}=v_{i}^{-1}\left(x_{0}\right) \omega_{0}\left(\partial_{i}\left(x_{0}\right)\right)$ for $1 \leq i \leq n$, there exists a unique solution $\left(\varphi, \gamma_{1}, \ldots, \gamma_{n}\right)$ of (104) such that $\varphi\left(x_{0}\right)=\varphi_{0}$ and $\gamma_{i}\left(x_{0}\right)=\gamma_{i}^{0}$ for all $1 \leq i \leq n$. By Proposition 61, $\omega=d \varphi$ satisfies (100) and $\omega\left(\partial_{i}\right)=v_{i} \gamma_{i}$ for $1 \leq i \leq n$.

By the assumption on $\left(\varphi_{0}, \omega_{0}\right)$, it follows from Proposition 45 that the unique solution $X=\Omega^{0}$ of the Lyapunov equation $X^{t} P+P^{t} X^{t}+T \rho_{0}=0$, with $T=-P^{t}-\left(P^{t}\right)^{-1}$ and $\rho_{0}=P^{t} \omega_{0} \omega_{0}^{t} P+c P^{t} \varphi_{0} \varphi_{0}^{t} P$, is invertible. Moreover, $2 \Omega_{s}^{0}=\mathcal{G}^{t}\left(x_{0}\right) \mathcal{G}\left(x_{0}\right)$ by Proposition 41. Now define $\beta \in \Gamma\left(V^{*} \otimes N_{f} M\right)$ by (98), and let $\Omega$ be the unique solution of (15) on $U$ such that $\Omega\left(x_{0}\right)=\Omega^{0}$. Shrinking $U$ if necessary, we may assume that $\Omega$ is invertible on $U$. By (17) and (103) we have

$$
\begin{aligned}
& d\left(\Omega^{t} P+P^{t} \Omega^{t}+T \rho\right)(X)=\Phi(X)^{t} \omega^{t} P+P^{t} \Phi(X)^{t} \omega^{t}-\left(\left(P^{t}\right)^{2}+I\right) d \omega(X) \omega^{t} P \\
& -\left(\left(P^{t}\right)^{2}+I\right) \omega d \omega^{t}(X) P-c\left(\left(P^{t}\right)^{2}+I\right) \omega(X) \varphi^{t} P-c\left(\left(P^{t}\right)^{2}+I\right) \varphi \omega(X)^{t} P=0 .
\end{aligned}
$$

Since (99) holds at $x_{0}$, it holds on $U$, hence $\tilde{f}=\mathcal{R}_{\varphi, \beta, \Omega}\left(\left.f\right|_{U}\right)$ is a $P$-transform of $\left.f\right|_{U}$.

Remark 64. Proposition 63 also holds for horizontal isometric immersions $f: M^{n}(c) \rightarrow$ $\mathbb{S}_{\epsilon}^{2 n+1}(c)$ with $c<0$ and $\epsilon=-1$ if $\varphi_{0} \in V$ and $\omega_{0} \in T_{x_{0}} M^{*} \otimes V$ are chosen so that the unique solution $X=\Omega^{0}$ of the Lyapunov equation $X^{t} P+P^{t} X^{t}+T \rho_{0}=0$, with $T=-P^{t}-\left(P^{t}\right)^{-1}$ and $\rho_{0}=P^{t} \omega_{0} \omega_{0}^{t} P+c P^{t} \varphi_{0} \varphi_{0}^{t} P$, is invertible. That such a pair $\left(\varphi_{0}, \omega_{0}\right)$ does exist was shown in part (ii) of Proposition 45. 
In the following corollary we summarize the process given by the $P$-transformation to generate a family of new horizontal isometric immersions $f: M^{n}(c) \rightarrow \mathbb{S}_{\epsilon}^{2 n+1}(c)$ starting with a given one and a vector-valued solution of a linear system of PDE's.

Corollary 65. Let $f: M^{n}(c) \rightarrow \mathbb{S}_{\epsilon}^{2 n+1}(c)$ be a horizontal isometric immersion with $\nu_{f} \equiv 0$, let $\left(u_{1}, \ldots, u_{n}\right)$ be local principal coordinates given by Corollary 58 , let $(v, h)$ the solution of (97) associated to $f$, let $P$ be an endomorphism of a Euclidean vector space $V$ such that $\sigma(P) \cap(-\sigma(P))=\emptyset$ and let $\left(\varphi, \gamma_{1}, \ldots, \gamma_{n}\right)$ be a $V$-valued solution of the linear system of PDE's

$$
\left\{\begin{array}{l}
i) \partial_{i} \varphi=v_{i} \gamma_{i}, \quad(i i) \partial_{i} \gamma_{j}=h_{j i} \gamma_{i}, 1 \leq i \neq j \leq n \\
i i i) \partial_{i} \gamma_{i}+\sum_{j \neq i} h_{j i} \gamma_{j}+\left(P^{t}\right)^{-1} \gamma_{i}+c v_{i} \varphi=0
\end{array}\right.
$$

on an open subset $W$ where

$$
\tilde{v}_{i}=v_{i}+\left\langle\gamma_{i},\left(P+P^{-1}\right) \Omega^{-1} \varphi\right\rangle
$$

does not vanish for $1 \leq i \leq n$, with $\Omega^{t}$ given by (75) for $C=\left(\left(P^{t}\right)^{2}+I\right)\left(d \varphi(d \varphi)^{t}+c \varphi \varphi^{t}\right) P$. Then $\tilde{f}: W \rightarrow \mathbb{S}_{\epsilon}^{2 n+1}(c)$ given by

$$
\begin{aligned}
\tilde{F}=i \circ \tilde{f}= & F-\sum_{j=1}^{n}\left(\left\langle\Omega^{-1} \varphi, \gamma_{j}\right\rangle+\left\langle P \Omega^{-1} \varphi, \gamma_{j}\right\rangle i\right) F_{*} X_{j} \\
& -\left(\left\langle\Omega^{-1} \varphi, \varphi\right\rangle+\left\langle P \Omega^{-1} \varphi, \varphi\right\rangle i\right) c F
\end{aligned}
$$

where $X_{j}=v_{j}^{-1} \partial_{j}$ for $1 \leq j \leq n, i: \mathbb{S}_{\epsilon}^{2 n+1}(c) \rightarrow \mathbb{C}_{\epsilon}^{n+1}$ is the umbilical inclusion and $F=i \circ f$, defines a new horizontal isometric immersion whose induced metric has constant curvature c. Moreover, the solution of (97) associated to $\tilde{f}$ is $(\tilde{v}, \tilde{h})$, with

$$
\tilde{h}_{i j}=h_{i j}+\left\langle\gamma_{j},\left(P+P^{-1}\right) \Omega^{-1} \gamma_{i}\right\rangle .
$$

\section{A decomposition theorem for the $P$-transformation}

In this section we prove the following decomposition theorem for the $P$-transformation, for which Remarks 32 also apply.

Theorem 66. Let $f: M^{n}(0) \rightarrow \mathbb{R}^{2 n}$ (respectively, $f: M^{n}(c) \rightarrow \mathbb{S}^{2 n+1}(c)$ ) be a Lagrangian (respectively, horizontal) isometric immersion and let $\tilde{f}=\mathcal{R}_{\varphi, \beta, \Omega, P}(f): \tilde{M}^{n}(0) \rightarrow \mathbb{R}^{2 n}$ (respectively, $\mathcal{R}_{\varphi, \beta, \Omega, P}(f): \tilde{M}^{n}(c) \rightarrow \mathbb{S}^{2 n+1}(c)$ ) be a $P$-transform of $f$ such that $P=P_{1} \oplus P_{2}$ with respect to an orthogonal decomposition $V=V_{1} \oplus V_{2}$. Define $\varphi_{j}, \beta_{j}, \Omega_{i j}$ by (22), and $\bar{\varphi}_{i}, \bar{\beta}_{i}, \bar{\Omega}_{i i}$ by (23), $1 \leq i, j \leq 2$. Then $\left(\varphi_{j}, \beta_{j}, \Omega_{j j}, P_{j}\right)$ defines a $P_{j}$-transform $f_{j}$ of $f$ for $1 \leq j \leq 2,\left(\bar{\varphi}_{i}, \bar{\beta}_{i}, \bar{\Omega}_{i i}, P_{i}\right)$ a $P_{i}$-transform $f_{i j}$ of $f_{j}$ for $1 \leq i \neq j \leq 2$ and (24) holds. If, in addition, $f\left(M^{n}(0)\right) \subset \mathbb{S}^{2 n-1}$ and $\tilde{f}$ is a $P^{*}$-transform of $f$, then $f_{j}$ is a $P_{j}^{*}$-transform of $f$ and $f_{j i}$ is a $P_{j}^{*}$-transform of $f_{i}$ for $1 \leq i \neq j \leq 2$. 
Proof: We give the proof for horizontal isometric immersions $f: M^{n}(c) \rightarrow \mathbb{S}^{2 n+1}(c)$, the case of Lagrangian isometric immersions $f: M^{n}(0) \rightarrow \mathbb{R}^{2 n}$ being similar. By the assumption that $\tilde{f}=\mathcal{R}_{\varphi, \beta, \Omega, P}(f)$ is a $P$-transform of $f$, we have that $\beta$ and $\Omega$ satisfy (98) and (99), respectively. In particular, $\Omega$ is an invertible solution of the Lyapunov equation (99), hence $\operatorname{ker}\left(P^{c}-\alpha I\right) \cap \operatorname{ker}\left(\varphi^{t}\right)^{c} \cap \operatorname{ker}\left(\omega^{t}\right)^{c}=\{0\}$ for any eigenvalue $\alpha$ of $P$ by Proposition 45. Therefore $\operatorname{ker}\left(P_{j}^{c}-\alpha I\right) \cap \operatorname{ker}\left(\varphi_{j}^{t}\right)^{c} \cap \operatorname{ker}\left(\omega_{j}^{t}\right)^{c}=\{0\}$ for $1 \leq j \leq 2$, and hence also $\Omega_{j j}$ is invertible by Proposition 45. By Theorem $7,\left(\varphi_{j}, \beta_{j}, \Omega_{j j}\right)$ satisfies the conditions of Definition 5 for $1 \leq j \leq 2$.

Since $P=P_{1} \oplus P_{2}$, and hence $T=T_{1} \oplus T_{2}$, equations (98) and (99) are equivalent to $\beta_{j}=\left(\phi f_{*} \omega_{j}^{t}+\epsilon \sqrt{|c|} \xi_{f} \varphi_{j}^{t}\right) P_{j}$ and

$$
\Omega_{i j}^{t} P_{i}+P_{j}^{t} \Omega_{i j}^{t}+T_{j} \rho_{j i}=0,1 \leq i, j \leq 2,
$$

where $\rho_{j i}=P_{j}^{t} \omega_{j} \omega_{i}^{t} P_{i}+c P_{j}^{t} \varphi_{j} \varphi_{i}^{t} P_{i}$. In particular, $f_{j}=\mathcal{R}_{\varphi_{j}, \beta_{j}, \Omega_{j j}}(f)$ is a $P_{j}$-transform of $f, 1 \leq j \leq 2$.

Now by (84) we have

$$
T_{i} \Omega_{i j}=\Omega_{j i}^{t} T_{j}^{t}, \quad 1 \leq i, j \leq 2
$$

Using this we obtain

$$
\begin{aligned}
\bar{\Omega}_{i i}^{t} P_{i}+P_{i}^{t} \bar{\Omega}_{i i}^{t}+T_{i} \bar{\beta}_{i}^{t} \bar{\beta}_{i}= & \left(\Omega_{i i}^{t}-\Omega_{j i}^{t}\left(\Omega_{j j}^{-1}\right)^{t} \Omega_{i j}^{t}\right) P_{i}+P_{i}^{t}\left(\Omega_{i i}^{t}-\Omega_{j i}^{t}\left(\Omega_{j j}^{-1}\right)^{t} \Omega_{i j}^{t}\right) \\
& +T_{i}\left(\beta_{i}^{t}-\Omega_{i j} \Omega_{j j}^{-1} \beta_{j}^{t}\right)\left(\beta_{i}-\beta_{j}\left(\Omega_{j j}^{t}\right)^{-1} \Omega_{i j}^{t}\right) \\
= & \left(\Omega_{i i}^{t} P_{i}+P_{i}^{t} \Omega_{i i}^{t}+T_{i} \beta_{i}^{t} \beta_{i}\right)-\Omega_{j i}^{t}\left(\Omega_{j j}^{-1}\right)^{t} \Omega_{i j}^{t} P_{i}-P_{i}^{t} \Omega_{j i}^{t}\left(\Omega_{j j}^{-1}\right)^{t} \Omega_{i j}^{t} \\
& -T_{i} \beta_{i}^{t} \beta_{j}\left(\Omega_{j j}^{-1}\right)^{t} \Omega_{i j}^{t}-T_{i} \Omega_{i j} \Omega_{j j}^{-1} \beta_{j}^{t} \beta_{i}+T_{i} \Omega_{i j} \Omega_{j j}^{-1} \beta_{j}^{t} \beta_{j}\left(\Omega_{j j}^{-1}\right)^{t} \Omega_{i j}^{t} \\
= & -\Omega_{j i}^{t}\left(\Omega_{j j}^{-1}\right)^{t}\left(\Omega_{i j}^{t} P_{i}+T_{j} \beta_{j}^{t} \beta_{i}\right)-\left(P_{i}^{t} \Omega_{j i}^{t}+T_{i} \beta_{i}^{t} \beta_{j}\right)\left(\Omega_{j j}^{-1}\right)^{t} \Omega_{i j}^{t} \\
& +\Omega_{j i}^{t}\left(\Omega_{j j}^{-1}\right)^{t} T_{j} \beta_{j}^{t} \beta_{j}\left(\Omega_{j j}^{-1}\right)^{t} \Omega_{i j}^{t} \\
= & \Omega_{j i}^{t}\left(\left(\Omega_{j j}^{-1}\right)^{t} P_{j}^{t}+P_{j}\left(\Omega_{j j}^{-1}\right)^{t}+\left(\Omega_{j j}^{-1}\right)^{t} T_{j} \beta_{j}^{t} \beta_{j}\left(\Omega_{j j}^{-1}\right)^{t}\right) \Omega_{i j}^{t}=0 .
\end{aligned}
$$

Now let $\left\{X_{1}, \ldots, X_{n}\right\}$ be an orthonormal principal frame for $f$ and define $\xi_{1}, \ldots, \xi_{n}$ and $X_{j, 1}, \ldots, X_{j, n}$ by $\xi_{l}=\phi_{*} f_{*} X_{l}$ and $D_{j} X_{j, l}=X_{l}, 1 \leq l \leq n, 1 \leq j \leq 2$. Denote $\omega_{j}\left(X_{l}\right)=$ $\gamma_{j, l}$ and $\beta_{j}^{t}\left(\xi_{l}\right)=\beta_{j}^{l}$, and define $\bar{\gamma}_{i, l}=\bar{\omega}_{i} X_{j, l}$ and $\bar{\beta}_{i}^{l}=\bar{\beta}_{i}^{t}\left(\xi_{j, l}\right)$, where $\xi_{j, l}=\mathcal{P}_{j}\left(\xi_{l}-\right.$ $\left.\beta_{j}\left(\Omega_{j j}^{-1}\right)^{t}\left(L_{j}^{-1}\right)^{t} \beta_{j}^{l}\right)$. It follows from (54) that

$$
\bar{\gamma}_{i, l}=\omega_{i} D_{j} X_{j, l}-\Omega_{i j} \Omega_{j j}^{-1} \omega_{j} D_{j} X_{j, l}=\gamma_{i, l}-\Omega_{i j} \Omega_{j j}^{-1} \gamma_{j, l} .
$$

On the other hand, from (34), (99) and (108) we obtain

$$
\begin{aligned}
\bar{\beta}_{i}^{l} & =\left(\beta_{i}^{t}-\Omega_{i j} \Omega_{j j}^{-1} \beta_{j}^{t}\right) \mathcal{P}_{j}^{t} \mathcal{P}_{j}\left(\xi_{l}-\beta_{j}\left(\Omega_{j j}^{-1}\right)^{t}\left(L_{j}^{-1}\right)^{t} \beta_{j}^{l}\right) \\
& =\beta_{i}^{l}-\rho_{i j}\left(\Omega_{j j}^{-1}\right)^{t}\left(L_{j}^{-1}\right)^{t} \beta_{j}^{l}-\Omega_{i j} \Omega_{j j}^{-1} \beta_{j}^{l}+\Omega_{i j} \Omega_{j j}^{-1} \rho_{j j}\left(\Omega_{j j}^{t}\right)^{-1}\left(L_{j}^{-1}\right)^{t} \beta_{j}^{l} \\
& =P_{i}^{t} \gamma_{i, l}+\rho_{i j}\left(\Omega_{j j}^{t}\right)^{-1} T_{j} \gamma_{j, l}-\Omega_{i j}\left(\Omega_{j j}^{-1} P_{j}^{t}+\Omega_{j j}^{-1} \rho_{j j} T_{j}^{t} \Omega_{j j}^{-1}\right) \gamma_{j, l} \\
& =P_{i}^{t} \gamma_{i, l}+\rho_{i j} T_{j}^{t} \Omega_{j j}^{-1} \gamma_{j, l}+\Omega_{i j} P_{j} \Omega_{j j}^{-1} \gamma_{j, l}=P_{i}^{t} \gamma_{i, l}+\left(\rho_{i j} T_{j}^{t}+\Omega_{i j} P_{j}\right) \Omega_{j j}^{-1} \gamma_{j, l} \\
& =P_{i}^{t} \gamma_{i, l}-P_{i}^{t} \Omega_{i j} \Omega_{j j}^{-1} \gamma_{j, l}=P_{i}^{t} \bar{\gamma}_{i, l} .
\end{aligned}
$$


It follows that $\left(\bar{\varphi}_{i}, \bar{\beta}_{i}, \bar{\Omega}_{i i}, P_{i}\right)$ defines a $P_{i}$-transform of $f_{j}$ for $1 \leq i \neq j \leq 2$.

We now prove the last asssertion. If $\tilde{f}$ is a $P^{*}$-transform of $f$, then $\varphi+\sum_{l=1}^{n} v_{l} P^{t} \gamma_{l}=0$. Thus $\varphi_{j}+\sum_{l=1}^{n} v_{l} P_{j}^{t} \gamma_{j, l}=0$ for $1 \leq j \leq 2$, which already shows that $f_{j}$ is a $P_{j}^{*}$-transform of $f$. To prove that $f_{j i}$ is a $P_{j}^{*}$-transform of $f_{i}$ for $1 \leq j \leq 2$, we must show that

$$
\bar{\varphi}_{j}+\sum_{l=1}^{n} v_{i, l} P_{j}^{t} \bar{\gamma}_{j, l}=\bar{\varphi}_{j}+P_{j}^{t} \bar{\omega}_{j} \sum_{l=1}^{n} \partial_{l}=0 .
$$

Using (107) we obtain

$$
\begin{aligned}
P_{j}^{t} \bar{\omega}_{j} & =P_{j}^{t}\left(\omega_{j} D_{i}-\Omega_{j i} \Omega_{i i}^{-1} \omega_{i} D_{i}\right) \\
& =P_{j}^{t} \omega_{j} D_{i}-\Omega_{j i} \Omega_{i i}^{-1} P_{i}^{t} \omega_{i} D_{i}-\Omega_{j i} \Omega_{i i}^{-1} \rho_{i i} T_{i}^{t} \Omega_{i i}^{-1} \omega_{i} D_{i}+\rho_{j i} T_{i}^{t} \Omega_{i i}^{-1} \omega_{i} D_{i} .
\end{aligned}
$$

On the other hand, it follows from (52), (88) and $\omega_{i}^{t}(v)=\sum_{l=1}^{n}\left\langle\gamma_{i, l}, v\right\rangle X_{l}$ that

$$
\begin{aligned}
\sum_{l=1}^{n} \omega_{z} D_{i} \partial_{l} & =\sum_{l=1}^{n} v_{i, l} \gamma_{z, l} \\
& =\sum_{l=1}^{n}\left(v_{l}-\left\langle\gamma_{i, l}, T_{i}^{t} \Omega_{i i}^{-1} \varphi_{i}\right\rangle\right) \gamma_{z, l} \\
& =\sum_{l=1}^{n} v_{l} \gamma_{z, l}-\omega_{z} \omega_{i}^{t} T_{i}^{t} \Omega_{i i}^{-1} \varphi_{i} \\
& =\sum_{l=1}^{n} v_{l} \gamma_{z, l}-\left(P_{z}^{t}\right)^{-1} \rho_{z i} P_{i}^{-1} T_{i}^{t} \Omega_{i i}^{-1} \varphi_{i}, \quad z=i, j
\end{aligned}
$$

Thus, using (109) and (110) we obtain

$$
\begin{aligned}
\bar{\varphi}_{j}+P_{j}^{t} \bar{\omega}_{j} \sum_{l=1}^{n} \partial_{l} & =\varphi_{j}-\Omega_{j i} \Omega_{i i}^{-1} \varphi_{i}+P_{j}^{t} \sum_{l=1}^{n} v_{l} \gamma_{j, l}-\rho_{j i} P_{i}^{-1} T_{i}^{t} \Omega_{i i}^{-1} \varphi_{i} \\
& -\Omega_{j i} \Omega_{i i}^{-1} P_{i}^{t} \sum_{l=1}^{n} v_{l} \gamma_{i, l}+\Omega_{j i} \Omega_{i i}^{-1} \rho_{i i} P_{i}^{-1} T_{i}^{t} \Omega_{i i}^{-1} \varphi_{i} \\
& -\Omega_{j i} \Omega_{i i}^{-1} \rho_{i i} T_{i}^{t} \Omega_{i i}^{-1} \sum_{l=1}^{n} v_{l} \gamma_{i, l}+\Omega_{j i} \Omega_{i i}^{-1} \rho_{i i} T_{i}^{t} \Omega_{i i}^{-1}\left(P_{i}^{t}\right)^{-1} \rho_{i i} P_{i}^{-1} T_{i}^{t} \Omega_{i i}^{-1} \varphi_{i} \\
& +\rho_{j i} T_{i}^{t} \Omega_{i i}^{-1} \sum_{l=1}^{n} v_{l} \gamma_{i, l}-\rho_{j i} T_{i}^{t} \Omega_{i i}^{-1}\left(P_{i}^{t}\right)^{-1} \rho_{i i} P_{i}^{-1} T_{i}^{t} \Omega_{i i}^{-1} \varphi_{i} \\
= & \left(\rho_{j i}-\Omega_{j i} \Omega_{i i}^{-1} \rho_{i i}\right)\left(P_{i}^{-1} T_{i}^{t} \Omega_{i i}^{-1} P_{i}^{t}+T_{i}^{t} \Omega_{i i}^{-1}\right. \\
& \left.+T_{i}^{t} \Omega_{i i}^{-1}\left(P_{i}^{t}\right)^{-1} \rho_{i i} P_{i}^{-1} T_{i}^{t} \Omega_{i i}^{-1} P_{i}^{t}\right) \sum_{l=1}^{n} v_{l} \gamma_{i, l}=0,
\end{aligned}
$$

where we have used that $\rho_{i i}=-P_{i}^{t} \Omega_{i i}\left(T_{i}^{t}\right)^{-1}-\Omega_{i i} P_{i}\left(T_{i}^{t}\right)^{-1}$, as follows from (107).

\subsection{The Bianchi P-cube.}

Given $P_{1}, P_{2} \in \mathbb{R}$ with $P_{1} \neq \pm P_{2}$, we say that a Bianchi quadrilateral $\left\{f, f_{1}, f_{2}, f_{12}\right\}$ is a Bianchi $\left(P_{1}, P_{2}\right)$-quadrilateral of $n$-dimensional horizontal submanifolds with constant curvature $c$ of $\mathbb{S}^{2 n+1}(c)$ if $f: M^{n}(c) \rightarrow \mathbb{S}^{2 n+1}(c)$ is horizontal, $f_{i}$ is a $P_{i}$-transform of $f$ for $1 \leq i \leq 2$ and $f_{12}$ is a $P_{2}$-transform of $f_{1}$ and a $P_{1}$-transform of $f_{2}$. Similarly one defines a Bianchi $\left(P_{1}, P_{2}\right)$-quadrilateral of $n$-dimensional flat Lagrangian submanifolds of 
$\mathbb{R}^{2 n}$, as well as of $n$-dimensional flat Lagrangian submanifolds of $\mathbb{R}^{2 n}$ that are contained in $\mathbb{S}^{2 n-1} \subset \mathbb{R}^{2 n}$, in the latter case requiring $f_{i}$ to be a $P_{i}^{*}$-transform of $f$ for $1 \leq i \leq 2$ and $f_{12}$ to be a $P_{2}^{*}$-transform of $f_{1}$ and a $P_{1}^{*}$-transform of $f_{2}$. Below we state and prove a Bianchi-cube theorem for the first of these classes, analogous results being true for the others. We will need the following result of [5] (see also [16]).

Proposition 67. If $f_{i}=\mathcal{R}_{\varphi_{i}, \beta_{i}, \Omega_{i}, P_{i}}(f): M_{i}^{n}(c) \rightarrow \mathbb{S}^{2 n+1}(c), 1 \leq i \leq 2$, are $P_{i}$-transforms of $f: M^{n}(c) \rightarrow \mathbb{S}^{2 n+1}(c)$ with $P_{1} \neq \pm P_{2}$ and $\left[d \omega_{1}^{t}, d \omega_{2}^{t}\right]=0$, then there is a unique isometric immersion $\tilde{f}: \tilde{M}^{n}(c) \rightarrow \mathbb{S}^{2 n+1}(c)$ such that $\left\{f, f_{1}, f_{2}, \tilde{f}\right\}$ is a Bianchi $\left(P_{1}, P_{2}\right)$-quadrilateral.

Given $P_{1}, \ldots, P_{k} \in \mathbb{R}$, with $P_{i} \neq \pm P_{j}$ for all $1 \leq i \neq j \leq k$, we say that a Bianchi cube $\left(\mathcal{C}_{0}, \ldots, \mathcal{C}_{k}\right)$ is a Bianchi $\left(P_{1}, \ldots, P_{k}\right)$-cube of $n$-dimensional horizontal submanifolds with constant curvature $c$ of $\mathbb{S}^{2 n+1}(c)$ if $f: M^{n}(c) \rightarrow \mathbb{S}^{2 n+1}(c)$ is horizontal and, for all $1 \leq s \leq k-1$,

(i) Each $f_{\alpha_{s+1}} \in \mathcal{C}_{s+1}$ with $\alpha_{s+1}=\alpha_{s} \cup\left\{i_{j}\right\}$ is a $P_{i_{j}}$-transform of $f_{\alpha_{s}} \in \mathcal{C}_{s}$.

(ii) $\left\{f_{\alpha_{s-1}}, f_{\alpha_{s-1} \cup\left\{i_{l}\right\}}, f_{\alpha_{s-1} \cup\left\{i_{j}\right\}}, f_{\alpha_{s+1}}\right\}$ is a Bianchi $\left(P_{i_{l}}, P_{i_{j}}\right)$-quadrilateral when $\alpha_{s+1}=$ $\alpha_{s-1} \cup\left\{i_{l}, i_{j}\right\}$.

Theorem 68. Let $f: M^{n}(c) \rightarrow \mathbb{S}^{2 n+1}(c)$ be a horizontal isometric immersion with $\nu_{f} \equiv 0$ and let $f_{i}=\mathcal{R}_{\varphi_{i}, \beta_{i}, \Omega_{i}, P_{i}}(f): M^{n}(c) \rightarrow \mathbb{S}^{2 n+1}(c), 1 \leq i \leq k$, be $P_{i}$-transforms of $f$ such that $P_{i} \neq \pm P_{j}$ and $\left[d \omega_{i}^{t}, d \omega_{j}^{t}\right]=0$ for all $1 \leq i \neq j \leq k$. Then there exists a unique Bianchi $\left(P_{1}, \ldots, P_{k}\right)$-cube $\left(\mathcal{C}_{0}, \ldots, \mathcal{C}_{k}\right)$ such that $\mathcal{C}_{0}=\{f\}$ and $\mathcal{C}_{1}=\left\{f_{1}, \ldots, f_{k}\right\}$.

Proof: We first prove existence. Since $f_{i}$ is a $P_{i}$-transform of $f$, we have

$$
\beta_{i}=P_{i}\left(\phi f_{*} \nabla \varphi_{i}+\epsilon \sqrt{|c|} \varphi_{i} \xi_{f}\right), \quad 1 \leq i \leq k .
$$

Define $\varphi: M^{n}(c) \rightarrow \mathbb{R}^{k}, \beta \in \Gamma\left(\left(\mathbb{R}^{k}\right)^{*} \otimes N_{f} M\right)$ and $\Omega \in \Gamma\left(\left(\mathbb{R}^{k}\right)^{*} \otimes \mathbb{R}^{k}\right)$ by

$$
\varphi=\left(\varphi_{1}, \ldots, \varphi_{k}\right), \quad \beta=\sum_{i=1}^{k} e^{i} \otimes \beta_{i} \text { and } \Omega=\sum \Omega_{i j} e^{j} \otimes e_{i}
$$

where $e_{1}, \ldots, e_{k}$ is the canonical basis of $\mathbb{R}^{k}, e^{1}, \ldots, e^{k}$ is its dual basis, and

$$
\Omega_{i j}=\frac{\left(1+P_{j}^{2}\right)}{P_{j}\left(P_{i}+P_{j}\right)} \rho_{i j}, 1 \leq i, j \leq k,
$$


with $\rho_{i j}=P_{i} P_{j}\left\langle\nabla \varphi_{i}, \nabla \varphi_{j}\right\rangle+c P_{i} P_{j} \varphi_{i} \varphi_{j}$. We claim that $(\varphi, \beta, \Omega)$ defines a $P$-transformation of $f$, where $P \in\left(\mathbb{R}^{k}\right)^{*} \otimes \mathbb{R}^{k}$ is given by $P=\sum_{i=1}^{k} P_{i} e^{i} \otimes e_{i}$. First notice that $f_{i}$ is an $L_{i}$-transform of $f$ for $1 \leq i \leq k$, with $L_{i}=\frac{P_{i}^{2}}{P_{i}^{2}+1}$, and that

$$
\Omega_{i j}=\frac{-L_{i}\left\langle\mathcal{G}_{i}, \mathcal{G}_{j}\right\rangle+\rho_{i j}}{L_{j}-L_{i}}
$$

where $\mathcal{G}_{i}=F_{*} \nabla \varphi_{i}+i_{*} \beta_{i}+c \varphi_{i} F$, with $F=i \circ f$. It follows from the proof of Theorem 34 that $(\varphi, \beta, \Omega)$ defines an $L$-transformation of $f$, where $L=\left(P^{2}+I\right)^{-1} P^{2}$. Equation (98) follows immediately from (111). It remains to prove (99), which is equivalent to

$$
\Omega_{i j}\left(P_{i}+P_{j}\right)+T_{j} \rho_{i j}=0,1 \leq i, j \leq k .
$$

where $T_{j}=-\left(P_{j}^{2}+1\right) / P_{j}$. For $i=j$ this follows from the fact that $f_{i}$ is a $P_{i}$-transform of $f$, while for $i \neq j$ this is a consequence of (112).

Now for any multi-index $\alpha_{r}=\left\{i_{1}, \ldots, i_{r}\right\} \in \Lambda_{r}$, define $\left(\varphi^{\alpha_{r}}, \beta^{\alpha_{r}}, \Omega^{\alpha_{r}}\right)$ by (67) and set $P^{\alpha_{r}}=\sum_{\ell, j=1}^{r} P_{i_{\ell} i_{j}} e^{i_{\ell}} \otimes e_{i_{j}}$. By Theorem 66, for each $\alpha_{r} \in \Lambda_{r}$ the triple $\left(\varphi^{\alpha_{r}}, \beta^{\alpha_{r}}, \Omega^{\alpha_{r}}\right)$

defines a $P^{\alpha_{r}}$-transform of $f$. Let $\mathcal{C}_{r}$ be the family of $\left(\begin{array}{l}k \\ r\end{array}\right)$ elements formed by the $P^{\alpha_{r}}$

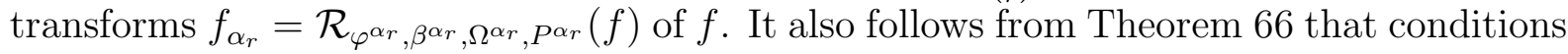
(i) and $(i i)$ in the definition of a Bianchi $\left(P_{1}, \ldots, P_{k}\right)$-cube are satisfied by $\left(\mathcal{C}_{0}, \ldots, \mathcal{C}_{k}\right)$.

Finally, since $\left(\mathcal{C}_{0}, \ldots, \mathcal{C}_{k}\right)$ is also a Bianchi $\left(L_{1}, \ldots, L_{k}\right)$-cube, the uniqueness assertion follows from that in Theorem 34, once we show that the last assumption in that result is unnecessary in this case. In fact, in view of Remark 35, if $f_{i}$ belongs to the associated family determined by $\left\{f_{j}, f_{l}\right\}, 1 \leq i \neq j \neq l \neq i \leq k$, then the vector field $\xi$ given by (68) satisfies $A_{\xi}=0$. Using (98) and the first equation in (89), this implies that $A_{\eta}=0$ for

$$
\eta=\phi f_{*}\left(k_{j}\left(C_{i}-C_{j}\right) P_{j} \nabla \varphi_{j}+k_{l}\left(C_{i}-C_{l}\right) P_{l} \nabla \varphi_{l}\right) \in \Gamma\left(\phi f_{*} T M\right) .
$$

But then the vector field under brackets must be zero by the second equation in (89) and the assumption that $\nu_{f} \equiv 0$, and this is a contradiction.

\section{Examples}

In this section we illustrate the procedures developed in the preceding sections. We produce examples of $n$-dimensional flat Lagrangian submanifolds of $\mathbb{C}^{n}$ by applying the $P$-transformation to the trivial solution

$$
v_{1}=1, \quad v_{i}=0, \quad 2 \leq i \leq n, \quad \text { and } \quad h_{i j}=0, \quad 1 \leq i \neq j \leq n,
$$

of the system of PDE's (71). 
Note that, given a solution $(v, h)$ of (71), with $v=\left(v_{1}, \ldots, v_{n}\right)$ and $h=\left(h_{i j}\right)$, on an open simply connected subset $U \subset \mathbb{R}^{n}$ where $v_{i} \neq 0$ everywhere for $1 \leq i \leq k$, in order to determine the corresponding Lagrangian isometric immersion $f: U \rightarrow \mathbb{C}^{n}$ one has to integrate the system of PDE's

$$
\left\{\begin{array}{l}
\text { i) } \partial_{i} f=v_{i} X_{i}, \quad \text { ii) } \partial_{j} X_{i}=h_{i j} X_{j}, \quad i \neq j, \\
\text { iii) } \partial_{i} X_{i}=-\sum_{k \neq i} h_{k i} X_{k}+i X_{i},
\end{array}\right.
$$

with initial conditions $\left(X_{1}\left(u_{0}\right), \ldots, X_{n}\left(u_{0}\right)\right)$ at some point $u_{0} \in U$ chosen so that

$$
\left\langle X_{i}\left(u_{0}\right), X_{j}\left(u_{0}\right)\right\rangle=\left\langle i X_{i}\left(u_{0}\right), X_{j}\left(u_{0}\right)\right\rangle=0, i \neq j, \text { and }\left\langle X_{i}\left(u_{0}\right), X_{i}\left(u_{0}\right)\right\rangle=1 .
$$

For the trivial solution (113), system (114) becomes

$$
\left\{\begin{array}{l}
\text { i) } \partial_{1} f=X_{1}, \quad \text { ii) } \partial_{i} f=0, \quad 2 \leq i \leq n \\
\text { iii) } \left.\partial_{j} X_{i}=0, \quad 1 \leq i \neq j \leq n, \quad i v\right) \partial_{i} X_{i}=i X_{i}, \quad 1 \leq i \leq n,
\end{array}\right.
$$

whose solution, with initial conditions $\left(F(0), X_{1}(0), \ldots, X_{n}(0)\right)=\left(-i E_{1}, E_{1}, \ldots, E_{n}\right)$, where $E_{1}, \ldots, E_{n}$ is the canonical basis of $\mathbb{C}^{n}$ over $\mathbb{C}$, is

$$
f=-i e^{i u_{1}} E_{1}, \quad X_{j}=e^{i u_{j}} E_{j}, \quad 1 \leq j \leq n .
$$

System (82) reduces in this case to

$$
\left\{\begin{array}{l}
\text { i) } \partial_{1} \varphi=\gamma_{1}, \quad \partial_{i} \varphi=0, \quad 2 \leq i \leq n \\
\text { ii) } \left.\partial_{i} \gamma_{i}=-\left(P^{t}\right)^{-1} \gamma_{i}, \quad i i i\right) \partial_{i} \gamma_{j}=0, j \neq i .
\end{array}\right.
$$

Given $P_{1}, \ldots, P_{k} \in \mathbb{R}$, with $P_{i} \neq \pm P_{j}$ for $1 \leq i \neq j \leq k$, the solution of (116) for the scalar $P_{i}$-transformation, $1 \leq i \leq k$, with initial conditions $\varphi_{i}(0)=-P_{i}$ and $\gamma_{j}^{i}(0)=1$, is

$$
\varphi_{i}=-P_{i} e^{-\frac{u_{1}}{P_{i}}} \text { and } \gamma_{j}^{i}=e^{-\frac{u_{j}}{P_{i}}}, \quad 1 \leq i \leq k, \quad 1 \leq j \leq n
$$

The corresponding $P_{i}$-transform of $f$ is

$$
f_{i}=-i e^{i u_{1}} E_{1}+\frac{2 P_{i}\left(1+P_{i} i\right) e^{-u_{1} / P_{i}}}{\left(1+P_{i}^{2}\right) \sum_{j=1}^{n} e^{-2 u_{j} / P_{i}}} \sum_{j=1}^{n} e^{\frac{\left(-1+P_{i} i\right) u_{j}}{P_{i}}} E_{j} .
$$

The remaining $2^{k}-(k+1)$ vertices of the Bianchi $\left(P_{1}, \ldots, P_{k}\right)$-cube $\left(\mathcal{C}_{0}, \ldots, \mathcal{C}_{k}\right)$, with $\mathcal{C}_{0}=\{f\}$ and $\mathcal{C}_{1}=\left\{f_{1}, \ldots, f_{k}\right\}$, are given by explicit algebraic formulae as follows. Let

$$
\Omega_{i j}=\frac{P_{i}\left(1+P_{j}^{2}\right)}{P_{i}+P_{j}} \sum_{\ell=1}^{n} e^{-\left(\frac{P_{i}+P_{j}}{P_{i} P_{j}}\right) u_{\ell}}, \quad 1 \leq i, j \leq k,
$$


and

$$
\mathcal{F}_{i}=\sum_{j=1}^{n}\left(1+P_{i} i\right) \gamma_{j}^{i} e^{i u_{j}} E_{j}, \quad 1 \leq i \leq k .
$$

Denote by $e_{1}, \ldots, e_{k}$ the canonical basis of $\mathbb{R}^{k}$, by $e^{1}, \ldots, e^{k}$ its dual basis, and for each $\alpha_{r}=\left\{i_{1}, \ldots, i_{r}\right\} \in \Lambda_{r}$ define

$$
\varphi^{\alpha_{r}}=\sum_{j=1}^{r} \varphi_{i_{j}} e_{i_{j}}, \Omega^{\alpha_{r}}=\sum_{\ell, j=1}^{r} \Omega_{i_{\ell} i_{j}} e^{i_{j}} \otimes e_{i_{\ell}} \text { and } \mathcal{F}^{\alpha_{r}}=\sum_{j=1}^{r} e^{i_{j}} \otimes \mathcal{F}_{i_{j}} .
$$

Then all elements of $\mathcal{C}_{r}, 2 \leq r \leq k$, are given by

$$
f_{\alpha_{r}}=f-\mathcal{F}^{\alpha_{r}}\left(\Omega^{\alpha_{r}}\right)^{-1} \varphi^{\alpha_{r}}, \quad \alpha_{r}=\left\{i_{1}, \ldots, i_{r}\right\} \in \Lambda_{r} .
$$

Note that, since the trivial solution $(v, h)$ we started with satisfies $\sum_{i=1}^{n} v_{i}^{2}=1$, and the corresponding solutions $\left(\varphi_{i}, \gamma_{1}^{i}, \ldots, \gamma_{n}^{i}\right)$ of (82) given by (117) satisfy (87) for $1 \leq i \leq k$, all elements of the Bianchi $\left(P_{1}, \ldots, P_{k}\right)$-cube we just constructed are flat $n$-dimensional Lagrangian submanifolds of $\mathbb{C}^{n}$ that are contained in $\mathbb{S}^{2 n-1}$, and hence are the liftings, by the Hopf projection, of flat $(n-1)$-dimensional Lagrangian submanifolds of $\mathbb{C P}^{n-1}$.

The preceding examples are those that can be obtained by applying a sequence of scalar $P^{*}$-transformations starting from the degenerate submanifold (115), that is, those which can be obtained from (115) by applying a $P^{*}$-transformation for a diagonalizable $P$.

For a general $P \in\left(\mathbb{R}^{k}\right)^{*} \otimes \mathbb{R}^{k}$, the two last equations of (116) become ordinary differential equations whose solution, with initial conditions $\gamma_{i}(0)=v_{i}^{0} \in \mathbb{R}^{k}, 1 \leq i \leq n$, is

$$
\gamma_{i}\left(u_{i}\right)=\exp \left(-u_{i}\left(P^{t}\right)^{-1}\right) v_{i}^{0}, \quad 1 \leq i \leq n .
$$

Let $\Omega^{t} \in\left(\mathbb{R}^{k}\right)^{*} \otimes \mathbb{R}^{k}$ be given by (75) for $C=\left(\left(P^{t}\right)^{2}+I\right) \sum_{i=1}^{n} \gamma_{i} \gamma_{i}^{t} P$. By Corollary [54, the corresponding $P^{*}$-transform of (115) is

$$
\tilde{f}=-i e^{i u_{1}} E_{1}+\sum_{j=1}^{n}\left(a_{j}+i b_{j}\right) e^{i u_{j}} E_{j}
$$

where

$$
a_{j}=\left\langle\Omega^{-1} P^{t} \gamma_{1}, \gamma_{j}\right\rangle \text { and } b_{j}=\left\langle\Omega^{-1} P^{t} \gamma_{1}, P^{t} \gamma_{j}\right\rangle \text {. }
$$

For instance, for the endomorphism $P \in\left(\mathbb{R}^{2}\right)^{*} \otimes \mathbb{R}^{2}$ whose matrix with respect to the canonical basis $e_{1}, e_{2}$ of $\mathbb{R}^{2}$ is, say, $\left(\begin{array}{rr}1 & 1 \\ -1 & 1\end{array}\right)$, one obtains, for $v_{j}^{0}=e_{1}, 1 \leq j \leq n$, that

$$
\gamma_{j}\left(u_{j}\right)=e^{-u_{j} / 2}\left(\cos \left(u_{j} / 2\right), \sin \left(u_{j} / 2\right)\right), \quad 1 \leq j \leq n,
$$




$$
\begin{aligned}
\Omega & =\sum_{\ell=1}^{n} \frac{e^{-u_{\ell}}}{4} \Omega_{\ell}, \quad \text { with } \Omega_{\ell}=\left(\begin{array}{cc}
3+\cos u_{\ell}-2 \sin u_{\ell} & 1+2 \cos u_{\ell}+\sin u_{\ell} \\
-1+2 \cos u_{\ell}+\sin u_{\ell} & 3-\cos u_{\ell}+2 \sin u_{\ell}
\end{array}\right), \\
a_{j} & =\frac{4}{5} D^{-1} e^{-U_{j}}\left(\left(2 \cos V_{j}-4 \sin V_{j}\right) G+\sum_{\ell=1}^{n} e^{-u_{\ell}}\left(\sin \left(u_{\ell}-U_{j}\right)-3 \cos \left(u_{\ell}-U_{j}\right)\right)\right)
\end{aligned}
$$

and

$$
b_{j}=\frac{8}{5} D^{-1} e^{-U_{j}}\left(\left(3 \cos V_{j}-\sin V_{j}\right) G-\sum_{\ell=1}^{n} e^{-u_{\ell}}\left(\sin \left(u_{\ell}-U_{j}\right)+2 \cos \left(u_{\ell}-U_{j}\right)\right)\right),
$$

where $U_{j}=\left(u_{1}+u_{j}\right) / 2, V_{j}=\left(u_{1}-u_{j}\right) / 2, G=\sum_{\ell=1}^{n} e^{-u_{\ell}}$ and

$$
D=2 G^{2}-\sum_{\ell, j=1}^{n} e^{-\left(u_{\ell}+u_{j}\right)} \cos \left(u_{\ell}-u_{j}\right)
$$

Since $P$ is non-diagonalizable, this provides an example of a $P^{*}$-transform of (115) that can not be obtained by an iteration of scalar $P^{*}$-transformations.

In a similar way one can produce examples of isometric immersions $f: M^{n}(c) \rightarrow$ $\mathbb{Q}^{n+p}(\tilde{c})$ satisfying the assumptions of either of Propositions 1 or 2 by applying the $L$ transformation to the trivial solution (113) of either of systems (11) or (3), according to whether $c=\tilde{c}$ or $c \neq \tilde{c}$, respectively. One can also obtain examples of horizontal isometric immersions $f: M^{n}(c) \rightarrow \mathbb{S}_{\epsilon}^{2 n+1}(c)$ by applying the $P$-transformation to the trivial solution (113) of system (97).

\section{References}

[1] L. Bianchi, "Lezioni di Geometria Differenziale", Bologna, 1927.

[2] M. Dajczer, L. Florit and R. Tojeiro, The vectorial Ribaucour transformation for submanifolds and applications. Trans. Amer. Math. Soc. 359 (2007), 4977-4997.

[3] M. Dajczer and R. Tojeiro, Flat totally real submanifolds of $\mathbb{C P}^{n}$ and the symmetric generalized wave equation. Tohoku Math. J. (2) 47 (1995), no. 1, 117-123.

[4] M. Dajczer and R. Tojeiro, Isometric immersions and the generalized Laplace and elliptic sinh-Gordon equations. J. Reine Angew. Math. 467 (1995), 109-147.

[5] M. Dajczer and R. Tojeiro, The Ribaucour transformation for flat Lagrangian submanifolds. J. Geom. Anal. 10 (2000), no. 2, 269-280. 
[6] M. Dajczer and R. Tojeiro, An extension of the classical Ribaucour transformation. Proc. London Math. Soc. 85 (2002), no. 1, 211-232.

[7] M. Dajczer and R. Tojeiro, Commuting Codazzi tensors and the Ribaucour transformations for submanifolds. Result. Math. 44 (2003), no. 3-4, 258-278.

[8] Q. Hu and D. Cheng, The polynomial solution to the Sylvester matrix equation. Appl. Math. Lett. 19 (2006), no. 9, 859-864.

[9] V. Kučera, The matrix equation $A X+X B=C^{*}$. SIAM. J. Appl. Math. 26 (1974), 15-25.

[10] Q. P. Liu and M. Manas, Vectorial Ribaucour transformation for the Lamé equations. J. Phys. A. 31 (1998), 193-200.

[11] E. Ma, A finite series solution of the matrix equation $A X-X B=C$. SIAM J. Appl. Math. 14 (1966), 490-495.

[12] J. D. Moore, Submanifolds of constant positive curvature I. Duke Math. J. 44 (1977), 449-484.

[13] H. Reckziegel, A correspondence between horizontal submanifolds of Sasakian manifolds and totally real submanifolds of Kählerian manifolds, Topics in differential geometry, vol. I, II, North Holland 1988, pp. 1063-1081.

[14] O. Taussky and H. Zassenhaus, On the similarity transformation between a matrix and its transpose. Pacific J. Math. 9 (1959), 893-896.

[15] C-L. Terng and E. Wang, Transformation of flat Lagrangian immersions and Egoroff nets. Asian J. Math. 12 (2008), 99-119.

[16] R. Tojeiro, Lagrangian submanifolds of constant sectional curvature and their Ribaucour transformation. Bull. Belgian Math. Soc. - Simon Stevin 8 (2001), 29-46.

Universidade Federal de Mato Grosso Avenida Valdon Varjão 6390

78600-000 - Barra do Garças - Brazil

E-mail: dasigu@ufmt.br
Universidade Federal de São Carlos

Via Washington Luiz km 235

13565-905 - São Carlos - Brazil

E-mail: tojeiro@dm.ufscar.br 